\title{
Oral Anticoagulant Therapy
}

\section{Antithrombotic Therapy and Prevention of Thrombosis, 9th ed: American College of Chest Physicians Evidence-Based Clinical Practice Guidelines}

\author{
Walter Ageno, MD; Alexander S. Gallus, MBBS; Ann Wittkowsky, PharmD, FCCP; \\ Mark Crowther, MD; Elaine M. Hylek, MD, MPH; and Gualtiero Palareti, MD
}

\begin{abstract}
Background: The objective of this article is to summarize the published literature concerning the pharmacokinetics and pharmacodynamics of oral anticoagulant drugs that are currently available for clinical use and other aspects related to their management.

Methods: We carried out a standard review of published articles focusing on the laboratory and clinical characteristics of the vitamin $\mathrm{K}$ antagonists; the direct thrombin inhibitor, dabigatran etexilate; and the direct factor $\mathrm{Xa}$ inhibitor, rivaroxaban.

Results: The antithrombotic effect of each oral anticoagulant drug, the interactions, and the monitoring of anticoagulation intensity are described in detail and discussed without providing specific recommendations. Moreover, we describe and discuss the clinical applications and optimal dosages of oral anticoagulant therapies, practical issues related to their initiation and monitoring, adverse events such as bleeding and other potential side effects, and available strategies for reversal.

Conclusions: There is a large amount of evidence on laboratory and clinical characteristics of vitamin $\mathrm{K}$ antagonists. A growing body of evidence is becoming available on the first new oral anticoagulant drugs available for clinical use, dabigatran and rivaroxaban.
\end{abstract}

CHEST 2012; 141(2)(Suppl):e44S-e88S

\begin{abstract}
Abbreviations: $\mathrm{AC}=$ anticoagulation clinic; $\mathrm{AMS}=$ anticoagulation management service; aPTT $=$ activated partial thromboplastin time; $\mathrm{AUC}=$ area under the curve; $\mathrm{Cmax}=$ peak plasma concentration; $\mathrm{ECT}=$ ecarin clotting time; $\mathrm{HR}=$ hazard ratio; INR = international normalized ratio; ISI = international sensitivity index; $\mathrm{PCC}=$ prothrombin complex concentrate; $\mathrm{PE}=$ pulmonary embolism; $\mathrm{POC}=$ point of care; $\mathrm{PSM}=$ patient self-management; $\mathrm{PST}=$ patient self testing; $\mathrm{PT}=$ prothrombin time; $\mathrm{TCT}=$ thrombin clotting time; $\mathrm{TTR}=$ time in therapeutic range; $\mathrm{UC}=$ usual care; VKA = vitamin $\mathrm{K}$ antagonist; $\mathrm{VKOR}=$ vitamin $\mathrm{K}$ oxide reductase; $\mathrm{WHO}=$ World Health Organization
\end{abstract}

$\mathbf{F}^{\infty}$ or many decades, the vitamin $\mathrm{K}$ antagonists (VKAs) have been the only oral anticoagulant drugs available for clinical use for the primary and secondary prevention of venous and arterial thromboembolic events. VKAs have been consistently shown to be highly effective in many settings and are now used by millions of patients worldwide. Laboratory and clinical studies have contributed to understanding of the complex pharmacokinetics and pharmacodynamics of VKAs, their interactions, antithrombotic effects, and the risks associated with their use. Several studies have addressed the practical issues related to the management of patients on VKAs treatment, with particular focus on laboratory and clinical monitoring and on reversal strategies.

More recently, new oral anticoagulant drugs, namely the direct thrombin inhibitor dabigatran etexilate and the direct factor Xa inhibitor rivaroxaban, have been approved for clinical use in several countries. A growing body of laboratory and clinical data is becoming available to better understand the mechanisms of action and the optimal management of these new compounds. In this article we summarize the published literature concerning the pharmacokinetics and pharmacodynamics of all oral anticoagulant drugs that are currently available for clinical use and other aspects related to their management. 


\subsection{Vitamin K Antagonists}

\subsection{Pharmacology}

VKAs produce their anticoagulant effect by interfering with the cyclic interconversion of vitamin $\mathrm{K}$ and its 2,3 epoxide (vitamin $\mathrm{K}$ epoxide), thereby modulating the $\gamma$-carboxylation of glutamate residues (Gla) on the N-terminal regions of vitamin K-dependent proteins (Fig 1).1-8 The vitamin K-dependent coagulation factors II, VII, IX, and X require $\boldsymbol{\gamma}$-carboxylation for their procoagulant activity, and treatment with VKAs results in the hepatic production of partially carboxylated and decarboxylated proteins with reduced coagulant activity. ${ }^{9,10}$ Carboxylation is required for a calcium-dependent conformational change in coagulation proteins ${ }^{11-13}$ that promotes binding to cofactors on phospholipid surfaces. In addition, the VKAs inhibit carboxylation of the regulatory anticoagulant proteins $\mathrm{C}, \mathrm{S}$, and $\mathrm{Z}$ and thereby have the potential to be procoagulant. ${ }^{14}$ Although the anticoagulant effect of VKAs is dominant, a transient procoagulant effect may occur when baseline protein $\mathrm{C}$ and protein $\mathrm{S}$ levels are reduced due to the start of VKA therapy and the acute phase of a thrombotic event and before the balanced decrease of vitamin K-dependent clotting factor levels is achieved. Carboxylation requires the reduced form of vitamin $\mathrm{K}$ (vitamin $\mathrm{KH}_{2}$ ), a $\gamma$-glutamyl carboxylase, molecular oxygen, and $\mathrm{CO}_{2} \cdot{ }^{1}$ Vitamin K epoxide can be reused by reduction to $\mathrm{VKH}_{2}$. The oxidation-reduction reaction involves a reductase pair. The first, vitamin $\mathrm{K}$ epoxide reductase, is sensitive to VKA, whereas vitamin $\mathrm{K}$ reductase is less sensitive. ${ }^{1-3}$ Therefore, the anticoagulant

Revision accepted August 31, 2011.

Affiliations: From the University of Insubria (Dr Ageno), Varese, Italy; Flinders University (Dr Gallus), Adelaide, SA, Australia; the University of Washington (Dr Wittkowsky), Seattle, WA; McMaster University (Dr Crowther), St. Joseph's Hospital, Hamilton, ON, Canada; the Boston University School of Medicine (Dr Hylek), Boston, MA; and the University Hospital S. Orsola-Malpighi (Dr Palareti), Bologna, Italy.

Funding/Support: The Antithrombotic Therapy and Prevention of Thrombosis, 9th ed: American College of Chest Physicians Evidence-Based Clinical Practice Guidelines received support from the National Heart, Lung, and Blood Institute [R13 HL104758] and Bayer Schering Pharma AG. Support in the form of educational grants was also provided by Bristol-Myers Squibb; Pfizer, Inc; Canyon Pharmaceuticals; and sanofi-aventis US.

Disclaimer: American College of Chest Physician guidelines are intended for general information only, are not medical advice, and do not replace professional medical care and physician advice, which always should be sought for any medical condition. The complete disclaimer for this guideline can be accessed at http:// chestjournal.chestpubs.org/content/141/2_suppl/1S.

Correspondence to: Walter Ageno, MD, Department of Clinical Medicine, Ospedale di Circolo, Viale Borri 57, 21100 Varese, Italy; e-mail: walter.ageno@uninsubria.it

(C) 2012 American College of Chest Physicians. Reproduction of this article is prohibited without written permission from the American College of Chest Physicians (http://www.chestpubs.org/ site/misc/reprints.xhtml).

DOI: 10.1378/chest.11-2292 effect of the VKAs can be overcome by low doses of phytonadione (vitamin K) (Fig 1).

\subsection{Pharmacokinetics and Pharmacodynamics}

Warfarin is a racemic mixture of two optically active isomers, the $\mathrm{R}$ and $\mathrm{S}$ enantiomers. Warfarin is highly water soluble, is rapidly absorbed from the gastrointestinal tract, has high bioavailability, ${ }^{15,16}$ and reaches maximal blood concentrations about $90 \mathrm{~min}$ after oral administration. ${ }^{15,17}$ Racemic warfarin has a half-life of 36 to $42 \mathrm{~h}^{18}$ (R-warfarin $45 \mathrm{~h}$, S-warfarin $29 \mathrm{~h}$ ), circulates bound to plasma proteins (mainly albumin), and accumulates in the liver where the two enantiomers are metabolically transformed by different pathways (Fig 1). ${ }^{18}$ The S enantiomer of warfarin (2.7-3.8 times more potent than the $\mathrm{R}$ enantiomer) undergoes approximately $90 \%$ oxidative metabolism, primarily by the CYP2C9 enzyme of the cytochrome P450 system and to a lesser extent by CYP3A4. ${ }^{19}$ The less potent $\mathrm{R}$ enantiomer undergoes approximately $60 \%$ oxidative metabolism, primarily by two cytochrome P450 enzymes, CYP1A2 and CYP3A4, and to a lesser extent by CYP2C19. The remainder of the metabolism of both enantiomers involves reduction to diastereomeric alcohols. The relationship between the dose of warfarin and the response is modified by genetic and environmental factors that can influence the absorption of warfarin, its pharmacokinetics, and its pharmacodynamics.

Other available VKAs include acenocoumarol, phenprocoumon, and fluindione. Like warfarin, acenocoumarol and phenprocoumon also exist as optical isomers, but with different stereochemical characteristics. R-acenocoumarol has an elimination half-life of $9 \mathrm{~h}$, is primarily metabolized by CYP2C9 and CYP2C19, and is more potent than $\mathrm{S}$-acenocoumarol because of faster clearance of S-acenocoumarol, which has an elimination half-life of $0.5 \mathrm{~h}$ and is primarily metabolized by CYP2C9. ${ }^{20}$ Phenprocoumon is a much longer-acting agent, with both the R- and S-isomers having elimination half-lives of 5.5 days. Both are metabolized by CYP2C9, and S-phenprocoumon is 1.5 to 2.5 times more potent than R-phenprocoumon. ${ }^{21}$ Finally, fluindione is an indandione VKA with a mean half-life of $31 \mathrm{~h} .{ }^{22}$ Unlike warfarin, fluindione is not a chiral compound. ${ }^{22}$

\subsection{Interactions}

1.3.1 Genetic Factors: A number of point mutations in the gene coding for the CYP2C9 have been identified. ${ }^{23}$ These polymorphisms, the most common of which are CYP2C9*2 and CYP2C9*3, are associated with an impaired ability to metabolize S-warfarin, resulting in a reduction in S-warfarin clearance and, 

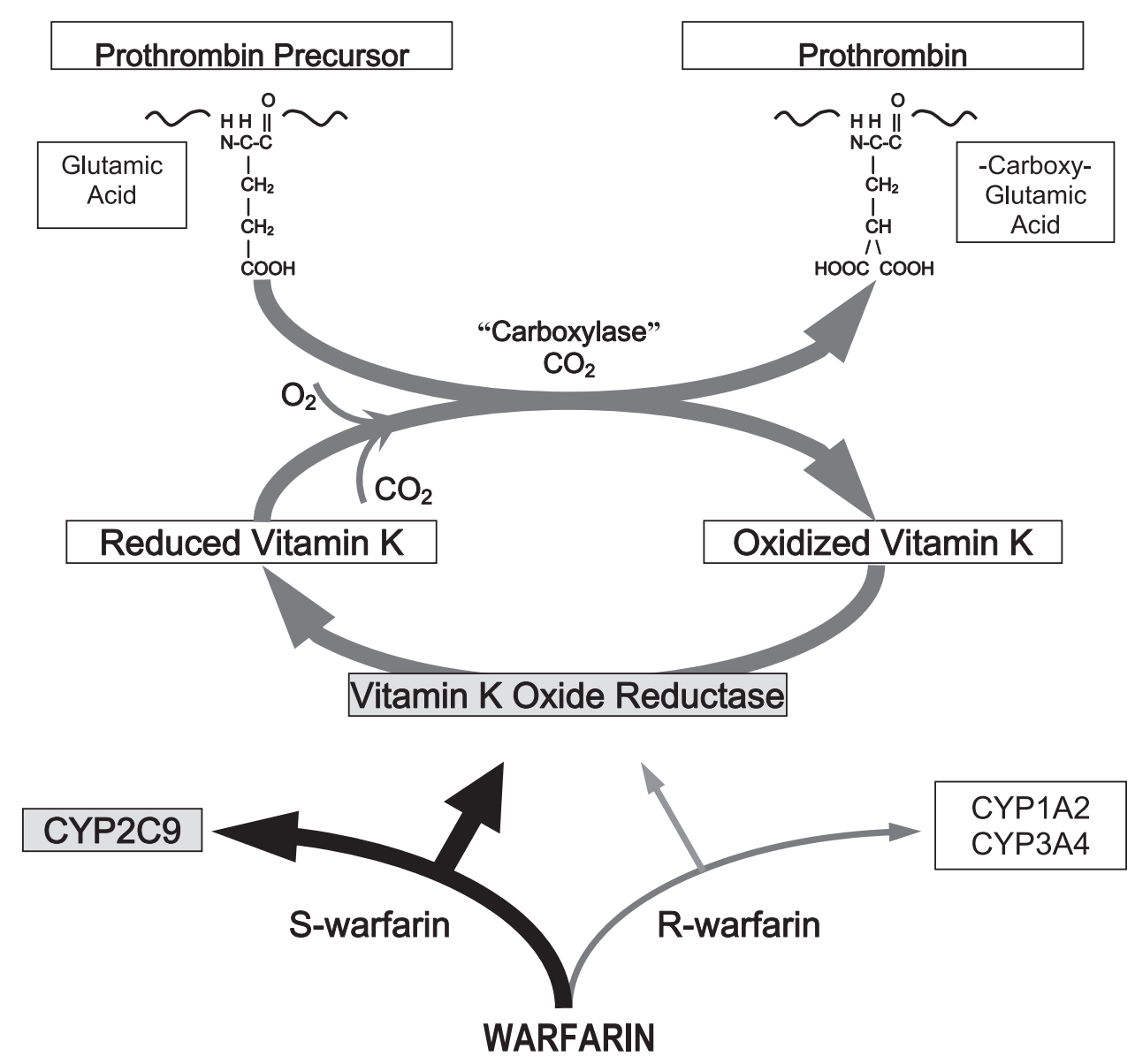

FiguRE 1. [Section 1.1] Vitamin $\mathrm{K}_{1}$ is reduced to vitamin KH2. The major warfarin-sensitive enzyme in this reaction is the vitamin $\mathrm{K}$ oxide reductase mainly inhibited by the $\mathrm{S}$-enantiomer of warfarin. S-warfarin is metabolized by the p450 cytochrome enzyme, CYP2C9. Reprinted with permission from Ansell et al. ${ }^{8}$

as a result, an increased S-warfarin elimination halflife. ${ }^{24}$ Mutations in this gene occur with different frequencies in various ethnic groups (Table S1).25,26 In comparison with patients who are homozygous for the wild-type allele (CYP2C $9 * 1 * 1)$, patients with heterozygous $(\mathrm{CYP} 2 \mathrm{C} 9 * 1 * 2$, CYP2C $9 * 1 * 3$, CYP2C $9 * 2 * 3$ ) or homozygous (CYP2C $9 * 2 * 2$, CYP2C $9 * 3 * 3$ ) expression of a variant allele require lower doses of warfarin, as determined by a systematic review of the literature and meta-analysis of studies that assessed the influence of CYP2C9 polymorphisms on warfarin dose requirements (Table S2). ${ }^{27}$ Several investigations ${ }^{25,28,29}$ have shown that these mutations, as well as others, ${ }^{30-32}$ are also associated with an increase in bleeding complications associated with warfarin therapy. Mutations in CYP2C9 also affect acenocoumarol, although to a lesser degree because the anticoagulation potencies of the $\mathrm{R}$ and $\mathrm{S}$ enantiomers are comparable. ${ }^{33,34}$ The effects of CYP2C9 polymorphisms are least pronounced with the use of phenprocoumon. ${ }^{33,35}$

The target for warfarin's inhibitory effect on the vitamin $\mathrm{K}$ cycle is the vitamin $\mathrm{K}$ oxide reductase
(VKOR) enzyme first described in 1974. ${ }^{36}$ The gene coding for the VKOR protein is located on the short arm of chromosome $16 .{ }^{37,38}$ The gene encodes for several isoforms of a protein that are collectively termed the vitamin $\mathrm{K}$ oxide reductase complex 1 (VKORC1). Subsequently, mutations in this gene have been identified leading to enzymes with varying sensitivities to inhibition by warfarin, ${ }^{38-43}$ thereby affecting the pharmacodynamics of warfarin. The mutations occur with differing frequencies in various ethnic populations and account, in part, for the difference in warfarin doses required to maintain a therapeutic international normalized ratio (INR) (Table S1) (tables that contain an " $\mathrm{S}$ " before the number denote supplementary tables not contained in the body of the article and available instead in an online data supplement; see the "Acknowledgments" for more information). $39-41,44,45$

Genetic mutations in the gene coding for the VKORCloften involve several mutations leading to various haplotypes that cause greater resistance to warfarin therapy. Harrington et $\mathrm{al}^{43}$ found a warfarinresistant individual who had high serum warfarin 
concentrations and a 196G $>$ A transition, predicting a Val66Met substitution in VKORC1. D'Andrea et al, ${ }^{39}$ studying 147 patients, found that those with a $1173 \mathrm{CC}$ genotype required a higher mean maintenance dose compared with those with a CT or TT genotype, as did Quiteineh et al, ${ }^{46}$ who found that a $1173 \mathrm{C}>\mathrm{T}$ polymorphism was significantly associated with the risk of anticoagulant overdose. By identifying a number of noncoding single nucleotide polymorphisms, Rieder et $\mathrm{al}^{40}$ were able to infer that there are five major haplotypes associated with different dose requirements for maintaining a therapeutic INR. The maintenance dose ranged from a low of $2.7 \mathrm{mg}$ warfarin per day for the sensitive haplotypes up to a high of $6.2 \mathrm{mg}$ per day for the resistant haplotypes. Asian Americans had the highest proportion of sensitive haplotypes, whereas African Americans more frequently exhibited the resistant haplotypes (Table S1).

1.3.2 Drugs: VKAs are highly susceptible to drug-drug interactions. For warfarin, for example, manufacturer-provided product information lists $>200$ specific agents that may interfere with this agent. ${ }^{47}$ Unfortunately, there seems to be little concordance among commonly used drug compendia and product labels with respect to interactions involving warfarin. Indeed, a major problem with the literature on this topic is that many reports are single-case reports and are not well documented. Anthony et $\mathrm{al}^{44}$ recently reviewed three drug information compendia, Clinical Pharmacology, ePocrates, and Micromedex, and the warfarin sodium (Coumadin) product label approved by the US Food and Drug Administration, for listings of interactions between warfarin and drugs, biologics, foods, and dietary supplements and found that of a total of 648 entries from the four sources, only 50 were common to all the sources. ${ }^{44}$ As in the previous edition of this article, ${ }^{8}$ Table 1 summarizes a comprehensive list of drugs that potentiate, inhibit, or have no effect on the anticoagulant effect of warfarin based on the results of a systematic review of available evidence completed in 2005, which rated warfarin drug interaction reports according to interaction direction, clinical severity, and quality of evidence, and developed lists of warfarin drug interactions considered highly probable, probable, possible, and highly improbable. 48

Drugs such as cholestyramine can reduce the anticoagulant effect of warfarin by reducing its absorption. Other drugs potentiate the anticoagulant effect of warfarin by inhibiting its clearance, whereas some drugs may inhibit the anticoagulant effect by enhancing its clearance. ${ }^{49}$ These latter effects may be through stereoselective or nonselective pathways, ${ }^{50,51}$ (stereoselective interactions may affect the oxida- tive metabolism of either the S-enantiomer or R-enantiomer of warfarin). The inhibition of S-warfarin metabolism is more important clinically, because this enantiomer is more potent than the R-enantiomer as a VKA. ${ }^{50,51}$ Phenylbutazone,${ }^{52}$ sulfinpyrazone, ${ }^{53}$ metronidazole, ${ }^{54}$ and trimethoprimsulfamethoxazole ${ }^{55}$ inhibit the clearance of $\mathrm{S}$-warfarin, and each potentiates the effect of warfarin on the prothrombin time (PT). In contrast, drugs such as cimetidine and omeprazole, which inhibit the clearance of the R-isomer, potentiate the PT only modestly in patients who are treated with warfarin.51,54,56 Amiodarone is a potent inhibitor of the metabolic clearance of both the S-enantiomer and the R-enantiomer and potentiates warfarin anticoagulation. ${ }^{57}$ The anticoagulant effect of warfarin is inhibited by drugs like barbiturates, rifampin, azathioprine, and carbamazepine, which increase its clearance by inducing hepatic metabolism. ${ }^{58}$ Azathioprine also reduces the anticoagulant effect of warfarin, presumably through a potentiating effect on hepatic clearance. ${ }^{59}$ Long-term alcohol consumption has a similar potential to increase the clearance of warfarin, but ingestion of even relatively large amounts of wine had little influence on the PT in normal volunteers who were given warfarin. ${ }^{60}$ The effect of enzyme induction on warfarin therapy has been analyzed in a critical review. ${ }^{58}$ Ten hepatic microsomal enzyme agents were assessed. Enzyme induction of warfarin metabolism by rifampin and barbiturates was considered likely, and an interaction with carbamazepine, griseofulvin, aminoglutethimide, nafcillin, and dicloxacillin was considered probable.

Drugs may also influence the pharmacodynamics of warfarin by inhibiting the synthesis of or increasing the clearance of vitamin K-dependent coagulation factors or by interfering with other pathways of hemostasis. The anticoagulant effect of warfarin is augmented by second-generation and third-generation cephalosporins, which inhibit the cyclic interconversion of vitamin $\mathrm{K}^{6}{ }^{61,62}$ by thyroxine, which increases the metabolism of coagulation factors; ${ }^{63}$ and by clofibrate through an unknown mechanism. ${ }^{64}$ Doses of salicylates of $>1.5$ g per day ${ }^{65}$ may augment the anticoagulant effect of warfarin. Acetaminophen potentiates the effect of warfarin when used over prolonged periods of time, as demonstrated in a recent randomized, blinded trial. ${ }^{66-68}$ Acetaminophen possibly potentiates the anticoagulant effect of warfarin through inhibition of VKOR by a toxic metabolite of the drug, ${ }^{69}$ although the accumulation of this metabolite may vary among individuals, thus accounting for a variable potentiating effect. ${ }^{70}$ Heparin potentiates the anticoagulant effect of warfarin, but in therapeutic doses produces only a slight prolongation of the PT. The mechanisms by which erythromycin ${ }^{71}$ and some anabolic steroids ${ }^{72}$ potentiate the anticoagulant effect of 


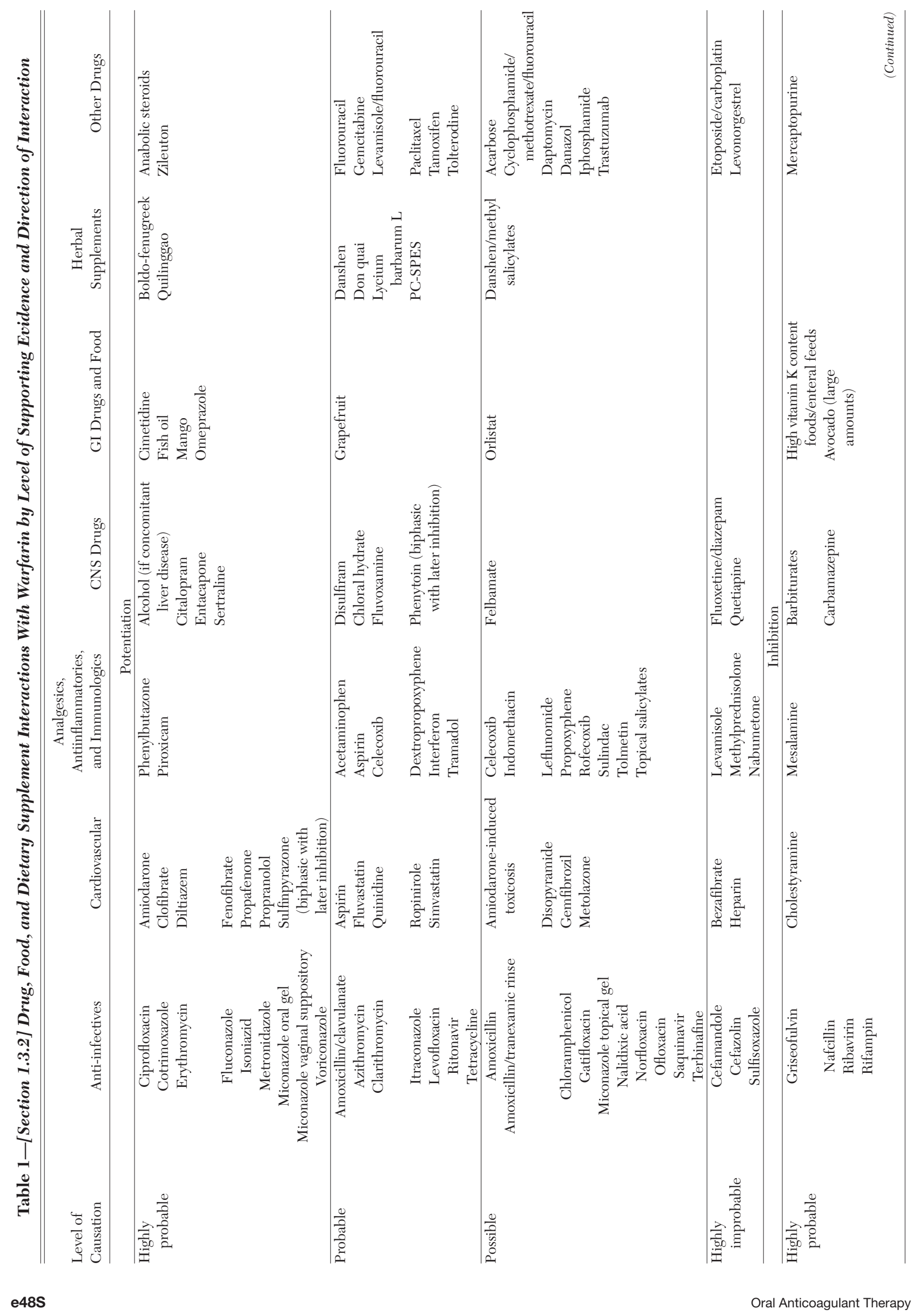




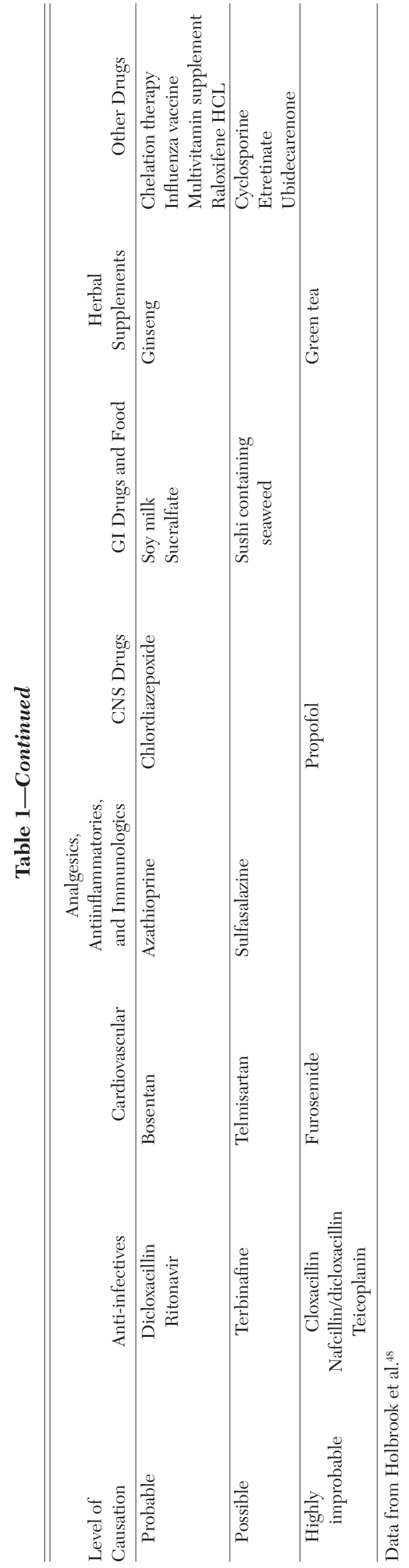

warfarin are unknown. Sulfonamides and several broadspectrum antibiotic compounds may augment the anticoagulant effect of warfarin in patients consuming diets that are deficient in vitamin $\mathrm{K}$ by eliminating bacterial flora and aggravating vitamin $\mathrm{K}$ deficiency. ${ }^{73}$

Drugs such as aspirin, ${ }^{74}$ nonsteroidal antiinflammatory drugs, ${ }^{75,76}$ penicillins in high doses, ${ }^{77,78}$ and moxalactam ${ }^{62}$ increase the risk of warfarin-associated bleeding by inhibiting platelet function. Of these, aspirin is the most important because of its widespread use and prolonged effect. ${ }^{79,80}$ Aspirin and nonsteroidal antiinflammatory drugs can also produce gastric erosions that increase the risk of upper GI bleeding. This can occur even with COX-2 inhibitors, which were originally believed to be less likely to predispose to gastric bleeding than nonsteroidal antiinflammatory drugs. $^{76}$ In one case-controlled analysis of 98,821 subjects on warfarin identified in linked databases, celecoxib and rofecoxib were associated with a 1.7 - or 2.4-fold increased risk of GI hemorrhage, respectively. ${ }^{76}$ The risk of clinically important bleeding is heightened when high doses of aspirin are taken during high-intensity warfarin therapy (INR, 3.0-4.5).74,81 However, low doses of aspirin (ie, 75 to $100 \mathrm{mg}$ daily) combined with moderate-intensity and low-intensity warfarin anticoagulation therapy are also associated with increased rates of bleeding. ${ }^{82,83}$ The effect of statins or fibrates on the risk of bleeding in patients on VKAs is controversial. The initiation of a fibrate or statin that inhibits CYP3A4 enzymes was recently reported to increase the risk of gastrointestinal bleeding in warfarin-treated patients, whereas other statins that are mainly excreted unchanged were not found to be associated with such an increased risk. ${ }^{84}$ Conversely, other authors reported that long-term statin use is associated with a decreased risk of bleeding complications in patients with atrial fibrillation $(\mathrm{AF})$ on warfarin therapy. ${ }^{85}$

The most effective method to avoid adverse outcomes associated with drug interactions is to try to avoid, when feasible, concurrent use of potentially interacting drugs and to use noninteracting alternatives instead. When noninteracting alternatives are not available, adverse outcomes can be avoided by increasing the frequency of monitoring and adjusting warfarin doses based on INR response. Prospective dosing adjustments are inappropriate because of the unpredictable nature of patient response to drug interactions (see Holbrook et al ${ }^{86}$ in this supplement).

1.3.3 Environmental Factors: Nutritional supplements and herbal products are particularly problematic in that warfarin-treated patients often fail to inform physicians that they are using such products and physicians rarely ask. In one survey of 1,200 patients from four large anticoagulation clinics (ACs) in the 
United States, one-third of the patients used dietary supplements and one-third of all patients surveyed indicated that their provider failed to discuss potential interactions with them. ${ }^{87}$ There is also little or no standardization of the content of such products, especially herbal remedies, and reports of interactions are often anecdotal or single-case reports, without good substantiation..$^{88-91}$ Of the higher-quality studies, ginkgo and ginger were shown not to have an effect on the pharmacokinetics and pharmacodynamics of warfarin in healthy subjects in a randomized, openlabel, crossover, study, ${ }^{92}$ and coenzyme $Q_{10}$ (and ginkgo) was shown not to have an effect on warfarin dosage in a randomized, double-blind, crossover study. ${ }^{93}$ Ginseng was shown to reduce the effect of warfarin in a randomized, placebo-controlled trial. ${ }^{94}$ Not surprisingly, products such as green tea, with a high content of vitamin $\mathrm{K}$, were shown to reduce the anticoagulant effect of warfarin. ${ }^{48}$

Subjects receiving long-term warfarin therapy are sensitive to fluctuating levels of dietary vitamin $\mathrm{K},{ }^{95,96}$ which is derived predominantly from phylloquinones in plant material. ${ }^{96}$ Sadowski and associates ${ }^{97}$ have listed the phylloquinone content of a wide range of food, and the list can be found on the Internet (http:// ods.od.nih.gov/factsheets/cc/coumadin1.pdf). Phylloquinones act through the warfarin-insensitive pathway. ${ }^{98}$ Important fluctuations in vitamin $\mathrm{K}$ intake can occur in both healthy and sick subjects. ${ }^{99}$ An increased intake of dietary vitamin $\mathrm{K}$ that is sufficient to reduce the anticoagulant response to warfarin occurs in patients consuming green vegetables or vitamin K-containing supplements, during weightreduction diets, and in patients who have been treated with vitamin $\mathrm{K}$ supplements. ${ }^{100}$ Reduced dietary vitamin $\mathrm{K}$ intake potentiates the effect of warfarin in ill patients who have been treated with antibiotics and IV fluids without vitamin $\mathrm{K}$ supplementation and in patients who have states of fat malabsorption.

In general, a consistent intake of vitamin Kcontaining foods is advisable, but neither specific restrictions nor additions seem necessary in patients with stable anticoagulant control. Patients should be informed of possible changes in INR, in particular in response to the use of dietary supplements or herbs, or alcohol used chronically or ingested in large quantities. ${ }^{101,102}$ More frequent monitoring of the INR should be proposed if dietary habits have substantially changed in response to weight reduction diets, periods following hospitalization, treatment with chemotherapy, sustained diarrhea or vomiting, or in case of anorexia. ${ }^{103}$

A number of other conditions and disease states have been observed to influence anticoagulation with warfarin. Hepatic dysfunction potentiates the response to warfarin through the impaired synthesis of coagulation factors. ${ }^{104}$ These patients may appear to be "auto-anticoagulated" with baseline elevated INRs, but the degree of suppression of clotting factors does not mimic that of patients treated with warfarin and is not sufficient to prevent thromboembolism. ${ }^{105}$ Hypermetabolic states produced by fever or hyperthyroidism increase warfarin responsiveness, probably by increasing the catabolism of vitamin K-dependent coagulation factors. ${ }^{70,106}$ Certain components of cigarette smoke may induce CYP1A2, and warfarin dosing requirements have been observed to decline after smoking cessation. ${ }^{107,108}$ Chewing tobacco contains high quantities of vitamin $\mathrm{K}$ that can increase warfarin dose requirements. ${ }^{109}$ Exacerbations of heart failure can increase responsiveness to warfarin therapy, likely in response to the effect of hepatic congestion on warfarin metabolism. ${ }^{110}$ Endstage renal disease is associated with reduced activity of CYP2C9, leading to lower warfarin dosing requirements in these patients. ${ }^{111}$ Warfarin dosing requirements decline with advanced age as a result of reduced availability of vitamin $\mathrm{K}$ stores and lower plasma concentrations of vitamin K-dependent clotting factors. ${ }^{112-114}$ In fact, age may be the single most important easily obtained predictor of warfarin dosing requirement. ${ }^{115}$

\subsection{Antithrombotic Effect}

The antithrombotic effect of VKAs is attributed to their anticoagulant effect, which in turn is mediated by the reduction of four vitamin K-dependent coagulation factors. The experiments of Wessler and Gitel $^{116}>40$ years ago using a stasis model of thrombosis in rabbits showed that the antithrombotic effect of warfarin requires 6 days of treatment and requires the reduction of prothrombin (factor II), which has a relatively long half-life of about 60 to $72 \mathrm{~h}$, compared with 6 to $24 \mathrm{~h}$ for other vitamin K-dependent factors. In a rabbit model of tissue factor-induced intravascular coagulation, the protective effect of warfarin mainly resulted from lowering prothrombin levels. ${ }^{117}$ Patel and associates ${ }^{118}$ demonstrated that clots formed from umbilical cord plasma containing about half the prothrombin concentration of plasma from adult control subjects generated significantly less fibrinopeptide A than clots formed from maternal plasma. The view that warfarin exerts its antithrombotic effect by reducing prothrombin levels is consistent with observations that clot-bound thrombin is an important mediator of clot growth ${ }^{119}$ and that reduction in prothrombin levels decreases the amount of thrombin generated and bound to fibrin, thereby reducing thrombogenicity. ${ }^{118}$

The suggestion that the antithrombotic effect of VKAs is reflected in lower levels of prothrombin forms the basis for overlapping the administration of 
a parenteral anticoagulant with warfarin until the PT or INR is prolonged into the therapeutic range during the treatment of patients with thrombosis. Since the half-life of prothrombin is about 60 to $72 \mathrm{~h}$, at least 5 days of overlap is necessary.

\subsection{Monitoring Anticoagulant Intensity: the INR}

The PT test ${ }^{120}$ is the most common test used to monitor VKA therapy. The PT responds to a reduction of three of the four vitamin K-dependent procoagulant clotting factors (ie, II, VII, and X) that are reduced by warfarin at a rate proportional to their respective half-lives. Thus during the first few days of warfarin therapy the PT reflects mainly a reduction of factor VII, the half-life of which is approximately $6 \mathrm{~h}$. Subsequently, the reduction of factors X and II contributes to prolongation of the PT. The PT assay is performed by adding calcium and thromboplastin to citrated plasma. Thromboplastins vary in responsiveness to a reduction of the vitamin $\mathrm{K}$-dependent coagulation factors. An unresponsive thromboplastin produces less prolongation of the PT for a given reduction in vitamin K-dependent clotting factors than a responsive one. The responsiveness of a thromboplastin can be measured by assessing its international sensitivity index (ISI) (see later discussion in this section). Highly sensitive thromboplastins (indicated by an ISI of approximately 1.0) are now available that are composed of human tissue factor produced by recombinant technology and defined phospholipid preparations.

PT monitoring of VKA treatment is not standardized when expressed in seconds, or as a simple ratio of the patient plasma value to that of plasma from a healthy control subject, or as a percentage of diluted normal plasma. A calibration model, ${ }^{121}$ which was adopted in 1982, is now used to standardize reporting by converting the PT ratio measured with the local thromboplastin into an INR, calculated as follows:

$$
\mathrm{INR}=(\text { patient } \mathrm{PT} / \text { mean normal PT })^{\mathrm{ISI}}
$$

or

$$
\log \mathrm{INR}=\mathrm{ISI}(\log \text { observed PT ratio })
$$

where ISI denotes the ISI of the thromboplastin used at the local laboratory to perform the PT measurement. The ISI reflects the responsiveness of a given thromboplastin to the reduction of the vitamin $\mathrm{K}$-dependent coagulation factors compared with the primary World Health Organization (WHO) international reference preparations, so that the more responsive the reagent, the lower the ISI value. ${ }^{121}$ It is proposed that patients' samples for calibration should be selected by rejecting samples beyond the 1.5 to 4.5 INR range. ${ }^{122}$ Outliers, defined as points with a perpendicular distance greater than three residual SDs from the line of relationship, should be rejected. Selection of patients' samples and rejection of outliers results in a reduction of the betweenlaboratory variation of calibration. ${ }^{122}$

As the INR standard of reporting was widely adopted, a number of problems surfaced. These are listed in Table 2 and are reviewed briefly here.

The INR is based on ISI values derived from the plasma of patients who had received stable anticoagulant doses for at least 6 weeks. ${ }^{123}$ As a result, the INR has not been validated and should be viewed with some skepticism early in the course of warfarin therapy, particularly when results are obtained from different laboratories. Even under these conditions,

\begin{tabular}{|c|c|}
\hline Problems & Description \\
\hline \multicolumn{2}{|l|}{ 1. Incorrect PTR from erroneous PT determination due to } \\
\hline Pretest variables (sampling and blood collection problems) & $\begin{array}{l}\text { Trisodium-citrate concentration, storage time, storage temperature, evacuated } \\
\text { tube effects, inadequate sample, variations in manual technique }\end{array}$ \\
\hline Incorrect normal value & $\begin{array}{l}\text { From nonuse of MNPT, error in MNPT due to unrepresentative selection, } \\
\text { technical faults (see above), nonuse of geometric mean }\end{array}$ \\
\hline $\begin{array}{l}\text { 2. Incorrect ISI of local thromboplastin reagent/test system } \\
\text { from lack of reliability of the ISI result provided by the } \\
\text { manufacturer }\end{array}$ & $\begin{array}{l}\text { Incorrect choice of IRP, poor distribution of coumarin test samples across } \\
\text { treatment range, inadequate numbers of test samples in ISI calibration, } \\
\text { incorrect transformation of PTR of test plasmas to INR }\end{array}$ \\
\hline \multicolumn{2}{|l|}{ 3. Drift of ISI since original calibration } \\
\hline \multicolumn{2}{|l|}{ 4. Instrument (coagulometer) effects on INR at local site } \\
\hline \multicolumn{2}{|l|}{ 5. Lupus anticoagulant effects on some thromboplastin reagents } \\
\hline \multicolumn{2}{|l|}{$\begin{array}{l}\text { 6. Lack of reliability of the INR system when used at the onset } \\
\text { of warfarin therapy and for screening for a coagulopathy } \\
\text { in patients with liver disease }\end{array}$} \\
\hline $\begin{array}{l}\text { 7. Relative lack of reliability of INR }>4.5 \text { as these values } \\
\text { excluded from ISI calibrations }\end{array}$ & \\
\hline
\end{tabular}

Table 2-[Section 1.5] Potential Problems With the INR (Causes of Erroneous INR) ${ }^{8}$

$\overline{\mathrm{INR}}=$ international normalized ratio; $\mathrm{IRP}=$ international reference preparation; $\mathrm{ISI}=$ international sensitivity index; $\mathrm{MNPT}=$ mean normal prothrombin time; $\mathrm{PT}=$ prothrombin time; $\mathrm{PTR}=$ prothrombin time ratio. 
however, the INR is more reliable than the unconverted PT ratio, ${ }^{124}$ and its use is thus recommended during both the initiation and maintenance of VKAs.

The validity of the INR in other conditions of impaired coagulation has been less frequently evaluated. Some authors have recently challenged the use of the INR in patients with liver disease and in particular in prognostic scores such as the Model for End-stage Liver Disease. ${ }^{125,126}$ Thus, a new INR specific for liver diseases has been proposed, derived by using plasma from patients with liver diseases to calibrate thromboplastins instead of plasma from patients on oral anticoagulant treatment with VKAs. ${ }^{125,126}$

The accuracy of the INR can be influenced by reagents with different sensitivities ${ }^{127}$ and also by the automated clot detectors now used in most laboratories. ${ }^{128-135}$ In general, the College of American Pathologists has recommended ${ }^{136}$ that laboratories should use thromboplastin reagents that are at least moderately responsive (ie, ISI $<1.7$ ) and reagent/instrument combinations for which the ISI has been established and validated.

ISI values provided by the manufacturers of thromboplastin reagents are not invariably correct when applied locally, ${ }^{137-139}$ and this adversely affects the reliability of measurements. Local calibrations can be performed using plasma samples with certified PT values to determine the instrument-specific ISI. The mean normal plasma PT is not interchangeable with a laboratory control PT. ${ }^{140}$ Therefore, the use of other than a properly defined mean normal PT can yield erroneous INR calculations, particularly when lessresponsive reagents are used. The mean normal PT should be determined for each new batch of thromboplastin with the same instrument used to assay the PT. ${ }^{140}$

The concentration of citrate that is used to anticoagulate plasma may affect the INR. ${ }^{141,142}$ In general, higher citrate concentrations (eg, 3.8\%) lead to higher INR values, ${ }^{141}$ and underfilling the blood collection tube spuriously prolongs the PT because excess citrate is present. Using collection tubes containing $3.2 \%$ concentrations of citrate for blood coagulation studies and adequately filling tubes can reduce this problem.

\subsection{Practical Issues Related to Initiation and Maintenance}

1.6.1 Approaches to the Induction of VKAs: Following the administration of VKAs, an initial effect on the INR usually occurs within the first 2 or 3 days, depending on the dose administered, and an antithrombotic effect occurs within the next several days. ${ }^{143,144}$ There is room for flexibility in selecting a starting dose of warfarin. The results of clinical studies suggest that initiation doses between 5 and $10 \mathrm{mg}$ are effective, ${ }^{143,145,146}$ with individual responses varying according to the inpatient or outpatient status, age, concomitant treatments, and comorbidities. Thus, starting doses of $\leq 5 \mathrm{mg}$ might be appropriate in the elderly, in patients with impaired nutrition, liver disease, or congestive heart failure, and in patients who are at high risk of bleeding. ${ }^{112,115,147,148}$ An initial dose of 2 to $3 \mathrm{mg}$ seems to be appropriate for patients who have undergone heart valve replacement, given their higher sensitivity to VKAs probably caused by the effects of cardiopulmonary bypass and concomitant therapies. ${ }^{149}$ Recommendations are provided in Holbrook et al. ${ }^{86}$

As described in section 1.2.1, CYP2C9 genotype and VKORC1 haplotype influence warfarin dosing requirements. In response to these observations, numerous investigators have attempted to assess the combined influence of patient factors on warfarin dosing requirement, with a goal of developing models to predict warfarin dose requirements based on assessment of various environmental and genetic factors.

Sconce et $\mathrm{al}^{42}$ found that a combination of CYP2C9 and VKORC1 genotypes plus height produced the best predictive model for estimating warfarin dose, whereas Vecsler et al ${ }^{150}$ reported that CYP2C9 and VKORC1 genotypes together with age and body weight could explain as much as $63 \%$ of the dose variance, and Herman et al ${ }^{151}$ could attribute $60 \%$ of dose variability to CYP2C9 and VKORClpolymorphisms, age, and body surface area. Limdi et al ${ }^{152}$ found that CYP2C9 and VKORC1 polymorphisms accounted for $30 \%$ of the variability in warfarin dose among European Americans but only for 10\% among African Americans. In a prospective cohort study of 48 consecutive patients starting warfarin for postorthopedic surgery prophylaxis, the patients with a variant CYP2C9 allele had a greater than fourfold increase in the risk of the INR exceeding 4.0. However, this substantial increase in the risk of supratherapeutic INR was observed despite the fact that all patients had their warfarin dose selected using a complex algorithm that considered CYP2C9 genotype. ${ }^{153}$

Gage et al $^{154}$ developed a dosing algorithm based on CYP2C9 and VKORC1 polymorphisms along with clinical and demographic factors. In the derivation cohort of 1,015 patients on warfarin therapy, body surface area, age, target INR, amiodarone use, smoker status, race, current thrombosis, VKORC1 polymorphism 1639/3673 G>A, CYP2C9(*)3, and CYP2C9(*)2 were all independent predictors of warfarin therapeutic dose.

Three prospective studies have compared the time in therapeutic range (TTR; see section 1.6.2 "Evaluating the Quality of Monitoring: TTR") of patients using a pharmacogenetics-based dosing strategy with the TTR achieved when patients were managed 
without knowledge of genotype. ${ }^{155-157}$ Two of these studies showed no difference in TTR, whereas one study reported increased times in range for the pharmacogenetic group but had significant design flaws. More recently, Klein et al ${ }^{158}$ developed two dosing algorithms: one based on clinical variables only and one based on both clinical variables and genetic information. The ability of these two algorithms to predict subsequent warfarin doses was then compared in a validation cohort of $>1,000$ patients. The pharmacogenetic algorithm more accurately identified patients who required $\leq 21 \mathrm{mg}$ weekly warfarin doses and patients who required weekly doses of $\geq 49 \mathrm{mg}$ in comparison with the clinical algorithm and to a fixeddose approach, whereas no difference was detected in the prediction of intermediate doses.

A few studies have suggested that certain genotypes are associated with adverse events. Thus, Higashi et al ${ }^{29}$ studied 185 patients, 58 with at least one variant genotype of CYP2C9, and found an increased risk of having INRs above range (hazard ratio [HR], 1.40; 95\% CI, 1.03-1.90) and of a serious or life-threatening bleeding event (HR, 2.39; 95\% CI, 1.18-4.86) in those with variant genotypes. The latter hazard estimate was based on a few events in a very small number of patients with the variant genotypes. Joffe et al, 159 also studying CYP2C9 single nucleotide polymorphisms, found a trend toward increased rates of an INR $>6.0$ and of bleeding in patients who were categorized as heterozygotes, or compound heterozygotes/homozygotes, compared with those categorized as wild type, as did Veenstra et al. ${ }^{30} \mathrm{~A}$ similar increased risk of bleeding was seen in patients with these polymorphisms who were taking acenocoumarol but not phenprocoumon. ${ }^{32}$ On the other hand, neither CYP2C9 nor VKORClinfluenced the risk of bleeding in a more recent study by Limdi et al. ${ }^{160}$

Likewise, the only high-quality, randomized, controlled trial performed to date showed that use of a pharmacogenetic-based dosing strategy did not significantly reduce the risk of adverse events $(34.7 \%$ in pharmacogenetic group vs $42.4 \%$ in control group; $\mathrm{OR}=0.72 ; 95 \% \mathrm{CI}, 0.41-1.28]) .155$ This issue is further discussed and recommendations are provided in Holbrook et al. ${ }^{86}$

When rapid anticoagulant effect is required, a rapidly acting parenteral anticoagulant should be started together with the VKA and discontinued after at least 5 days of concomitant therapy and once the INR has been in the therapeutic range for at least two measurements approximately $24 \mathrm{~h}$ apart. This allows factors $\mathrm{X}$ and II to be reduced to levels sufficient to treat VTE. If there is no urgent need for an immediate anticoagulant effect (eg, in chronic stable AF), warfarin administration can be commenced without the concurrent use of a rapid-acting anticoagulant.
1.6.2 Evaluating the Quality of Monitoring: TTR: The relationship between the intensity of treatment and the risk of an adverse event has been evaluated by examining the frequency of such events as a function of the TTR. ${ }^{161-163}$ A strong relationship between TTR and the rates of bleeding or thromboembolic events has been observed across studies ${ }^{161-173}$ with different patient populations, different target ranges, different scales for measuring intensity of anticoagulation (ie, PT, PT ratio, and INR), different methods of measuring TTR, and different models of dose management. In a large, retrospective analysis of patients with mechanical heart valves, Cannegieter et al ${ }^{164}$ found that risks of major bleeding or thromboembolism were greatly increased during the times when patients were above or below the therapeutic range of INR compared with times when they were within range. A similar relationship has been demonstrated for other groups of patients. ${ }^{169,174}$ A recent substudy examined the influence of TTR on the relative effectiveness of warfarin and dual antiplatelet therapy with aspirin plus clopidogrel in patients with nonvalvular $\mathrm{AF}$, in which the overall result favored warfarin. ${ }^{165}$ In this large retrospective analysis, the TTR during warfarin therapy appeared to be a major determinant of its efficacy, since the advantage of warfarin over antiplatelet therapy was lost below a threshold TTR of between $58 \%$ and $65 \%$. ${ }^{165}$ The percentage of INRs or TTR is highly dependent on the quality of dose management as reflected in studies that report TTR. Poor quality of dose management results in a high proportion of low INRs during the first 3 months of treatment following an acute DVT, which in turn predicts a higher rate of subsequent recurrence. ${ }^{161,175}$ The TTR reflects the quality of dose adjustment in studies of patients managed in a usual care (UC) setting, by an anticoagulation management service (AMS), by patient self testing (PST) or patient self management (PSM), or in the setting of a randomized trial.

TTR can be determined in different ways, so comparisons between studies may be difficult. ${ }^{176}$ TTR is most commonly estimated by using one of three methodologies: calculating the fraction of all INR values that are within the therapeutic range (ie, the number of INRs in range divided by the total number of INR tests); using the "cross-section of the files" methodology, which assesses the fraction of patients with an INR in range at one point in time compared with the total number of patients who had an INR measured at that point in time; or applying the linear interpolation method of Rosendaal et al, ${ }^{177}$ which assumes that a linear relationship exists between two INR values and allocates a specific INR value to each day between tests for each patient. Each approach has its advantages and disadvantages. ${ }^{176}$ Furthermore, the results of all these methods depend on whether 
an exact or an expanded therapeutic range is used, ${ }^{178}$ whether INRs obtained during invasive procedures when warfarin therapy might be interrupted are included, and whether different oral anticoagulant preparations (eg, warfarin, phenprocoumon, acenocoumarol, or fluindione) are included. ${ }^{179,180}$ Since clinical outcome studies have not compared one methodology with another and correlated their results with adverse events, no one method can be recommended, and the reader should be aware of these differences.

1.6.3 Frequency of Monitoring: In hospitalized patients, INR monitoring is usually performed daily until the therapeutic range has been achieved and maintained for at least 2 consecutive days. In outpatients starting VKA therapy, initial monitoring may be reduced to once every few days until a stable dose response has been achieved. When the INR response is stable, the frequency of testing can be reduced to intervals as long as every 4 to 6 weeks (or possibly longer in particularly stable patients). If adjustments to the dose are required, then the cycle of more frequent monitoring should be repeated until a stable dose response can again be achieved.

The optimal frequency of long-term INR monitoring is influenced by patient compliance, transient fluctuations in the severity of comorbid conditions, the addition or discontinuation of other medications, changes in diet, the quality of dose-adjustment decisions, and whether the patient has demonstrated a stable dose response. The dose required to maintain a therapeutic range for patients $>60$ years of age decreases with increasing age, ${ }^{112,115,181,182}$ possibly because of a reduction in the clearance of warfarin with age. ${ }^{183}$ Gender also influences dose, with women requiring less warfarin to maintain a therapeutic INR than men at an equivalent age. ${ }^{115}$

To compare different intervals between measurements of INR in stable patients, Pengo et al ${ }^{184}$ randomized 124 patients with prosthetic mechanical heart valves and at least 6 months of a steady dose requirement to INR monitoring at either 6 -week or 4 -week intervals. They found no differences of time in, above, or below range between the groups, although the actual monitoring intervals were 24.9 days in the 6 -week group and 22.5 days in the 4 -week group $(P<.0003) .{ }^{184}$ In a retrospective study of $>4,000$ patients with chronic $\mathrm{AF}$ and $>250,000$ INRs, Shalev et al ${ }^{185}$ found that time in range increased as the testing interval decreased from every 5 weeks or more to every 3 weeks ( $41 \%$ to $48 \%, P<.0005$ ), and the investigators suggested that patients should be monitored at time intervals no longer than every 3 weeks. However, the strength of the recommendation is reduced by the very poor TTR reported in both arms of the study. More recently, Witt et al 186,187 found that patients with very stable INR levels defined by extremely high TTR rates required fewer visits for INR control and had significantly fewer hemorrhagic and thromboembolic events than patients with a less-stable INR; they suggested that many warfarintreated patients whose INR values remain within the therapeutic range over time could be safely treated with INR recall intervals $>4$ weeks. In this study, advanced age predicted stable anticoagulation.

1.6.4 Factors Associated With INR Stability in Long-term Management: Two recent studies have assessed factors associated with very stable INR control during treatment with VKAs. ${ }^{186,187}$ In the first study, 2,504 patients with INR values entirely within the INR range for 6 months were compared with 3,569 patients with at least one INR value outside the INR range. ${ }^{186}$ In the second study, 533 patients with INR values within the therapeutic range for 12 months were compared with 2,555 control subjects. ${ }^{187}$ Independent predictors of stability were age $>70$ years, the absence of chronic diseases, and (in one study only ${ }^{187}$ ) male gender. Congestive heart failure, diabetes, and a target range for INR $\geq 3.0$ were associated with instability.

Physical activity also seems to play a role in the stability of the response to warfarin. A reduction in the anticoagulant effect has been found to be correlated with a sudden increase in physical activity. An increase in warfarin requirements associated with an increase in physical activity (represented by a daily exercise such as walking) has been described both in patients and in healthy subjects. ${ }^{188,189}$

Changes in dietary vitamin $\mathrm{K}$ intake may influence the stability of the INR in patients on VKAs, and a few trials have assessed its impact on therapeutic stability. Sorano et al ${ }^{190}$ showed that controlling the intake of dietary vitamin $\mathrm{K}$ can achieve a more stable anticoagulant response. Sconce et al, ${ }^{191}$ by comparing the daily vitamin $\mathrm{K}$ intake in 26 unstable patients and in 26 stable control patients, showed that unstable patients have poorer intake of vitamin K. Kurnik et al ${ }^{192}$ showed that in vitamin $\mathrm{K}$-depleted patients, very small amounts of vitamin K-containing vitamins will influence the INR to a greater extent compared with those with an adequate vitamin K status. Schurgers et al, ${ }^{193}$ studying healthy volunteers on oral anticoagulation, found that a daily dose of vitamin K of at least $150 \mu \mathrm{g}$ was needed to alter the INR response. Reese et al, ${ }^{194}$ in a retrospective analysis, assessed the effect of a daily dose of $100 \mu \mathrm{g}$ of vitamin $\mathrm{K}_{1}$ in nine unstable patients. These patients experienced an increase in the percentage of INRs in range from $32 \%$ to $57 \%$ in response to the daily vitamin $\mathrm{K}$. In a prospective, open-label, crossover study, Ford et al ${ }^{195}$ found that 
five of nine patients improved their stability with administration of low-dose vitamin K. As expected, the INR initially decreased in patients given vitamin $\mathrm{K}$, and an increased dose of warfarin was needed to reestablish an INR within the therapeutic range, which took from 2 to 35 days to achieve. In a nested casecontrol study, Rombouts et al ${ }^{196}$ assessed the effect of dietary vitamin $\mathrm{K}$ intake on the risk of subtherapeutic INR values and the interaction between usual and recent vitamin $\mathrm{K}$ intake. Patients with a high usual vitamin $\mathrm{K}$ intake had less risk of a subtherapeutic INR, an effect possibly mediated by a reduced influence on the INR of an incidental consumption of vitamin $\mathrm{K}$-rich food when there is a high usual intake of vitamin K. In these last two studies, variable INR was defined as requiring a minimum of three warfarin dose changes or three INRs outside of the therapeutic range in the preceding 6 months, ${ }^{195}$ or an INR $\mathrm{SD}>0.5$ with at least three warfarin dose changes during the previous 6 months. ${ }^{196}$

Sconce et al ${ }^{197}$ conducted the first randomized, blinded trial in 70 unstable patients over a 6 -month period. Vitamin K supplementation with $150 \mu \mathrm{g} / \mathrm{d}$ resulted in a significantly greater decrease in SD of the INR compared with placebo $(-0.24 \pm 0.14$ vs $-0.11 \pm 0.18 ; P<.001)$ and a significantly greater increase in percentage of time within target INR range $(28 \% \pm 20 \%$ vs $15 \% \pm 20 \% ; P<.01)$. Finally, Rombouts et al ${ }^{198}$ randomized 100 patients treated with phenprocoumon to receive daily doses of $100 \mu \mathrm{g}$ of vitamin $\mathrm{K}$ and 100 patients to receive a placebo. Vitamin K improved the stability of anticoagulant therapy, with a relative risk of maximal stability (where all INR results were in range) in the vitamin $\mathrm{K}$ group compared with the placebo group of 1.8 (95\% CI, 1.1-2.7).

De Assis et al ${ }^{199}$ randomized patients with a recent INR value outside the therapeutic range to a conventional approach based on changes in anticoagulant prescription or to a strategy that adjusted the dietary intake of vitamin K. Patients in the latter group had significantly more INR values within the therapeutic range and significantly fewer episodes of minor bleeding as compared with patients in the former group.

As with any other drug, a patient's nonadherence to prescribed dosing with VKAs is one of the most important causes of INR instability. In a prospective cohort study at three US ACs aimed to determine the effect of adherence on anticoagulation control, patients treated with warfarin were monitored with an electronic system that records each time they open their medication container. ${ }^{200}$ There was a high proportion of missed tablets and, as expected, in multivariable analyses there was a significant association between under-adherence and under-anticoagulation.
In a case-control study on the most unstable patients from 35 Italian ACs, poor comprehension of the indications and mechanisms of VKAs was the most important predictor of instability among other factors, including working status, type of VKA, and a poor score on a mental test. ${ }^{161}$ In a single-center study carried out on outpatients attending an AC in the United States, perceived barriers to compliance with warfarin, marital status, living arrangements, and drug regimen played significant roles in warfarin noncompliance. ${ }^{201}$ In a prospective cohort study of adults initiating warfarin at two ACs, independent risk factors for nonadherence were education level, employment status, mental health functioning, and cognitive impairment. ${ }^{202}$

\subsection{Data Management}

An obstacle to the safety and effectiveness of warfarin therapy is the poor quality of dose management in routine clinical practice. Adequate anticoagulant care with the VKAs requires a system of patient education and careful data management to record and track INR values and to ensure patients are treated with anticoagulants for an appropriate period of time.

1.7.1 The Role of Anticoagulation Clinics: Nonrandomized, retrospective studies have reported better outcomes in patients when anticoagulant therapy is managed by an AMS or an AC compared with management by their personal physicians (ie, UC). Four such studies have reported major bleeding rates ranging from $2.8 \%$ to $8.1 \%$ per patient-year of UC. ${ }^{203-206}$ Rates of thromboembolism with UC were not reported except in two studies in which the event rates were $6.2 \%$ and $8.1 \%$ per patient-year. Similarly, retrospective and prospective cohort studies of care provided by an AMS reported rates of major hemorrhage or thrombosis ranging from $1.4 \%$ to $3.3 \%$ and $0.7 \%$ to $6.3 \%$ per patient-year of therapy, respectively. ${ }^{164,170,207-210}$ Three retrospective comparative studies using a before-and-after design of patients managed by UC or an AMS reported significant improvements in the outcomes of hemorrhage or thrombosis with AMS-directed care..$^{211-213}$ In contrast, however, two prospective, randomized controlled trials ${ }^{214,215}$ comparing UC with the care of an AMS failed to show a significant difference in major hemorrhage or thromboembolism. The study by Matchar et al ${ }^{214}$ also failed to show a significant improvement in TTR, although the AMS performed modestly better than UC. Wilson et al ${ }^{215}$ did observe a significant improvement in TTR in the AMS group compared with UC ( $82 \%$ vs $76 \%$ respectively, $P=.034$ ). They also noted more high-risk INRs with UC than with an AMS ( $40 \%$ vs $30 \%, P=.005$ ). This latter study had a major 
limitation in that all patients were initially managed in an AMS for 3 months until they were stable and then observed for only 3 months after randomization to either receive UC or to continue care by the AMS. ${ }^{215}$ The other study ${ }^{214}$ suffered from a high turnover of patients, the possibility of selection bias affecting the referral of patients to the AMS, the open nature of the study, and targeted ranges that were sometimes outside recommended guidelines. In a retrospective, multicenter, international cohort study, 1,511 patients were randomly recruited from clinics offering routine medical care (UC) in the United States, Canada, and France, and from ACs in Italy and Spain. ${ }^{216}$ Major management differences were detected, especially between AC care and routine medical care, but also among countries. For patients managed by routine medical care, documentation of care was often inadequate. Finally, less time in therapeutic INR range was noted in routine medical care.

In a systematic review of 67 studies representing $>50,000$ patients managed by ACs $(68 \%)$, during clinical trials $(7 \%)$, or in community practices $(24 \%)$, van Walraven et $\mathrm{al}^{217}$ found that the practice setting had the greatest effect on anticoagulation control. TTR (days) varied from $56.7 \%$ in community practices to $65.6 \%$ in ACs and $66.4 \%$ for randomized trials. Compared with randomized trials the absolute reduction of TTR for community practices was $-12.2 \%$ (95\% CI, -19.5 to -4.8 ). The difference between community practices and ACs was $-8.3 \%$ (95\% CI, -4.4 to -12.1 ).

1.7.2. Computerized Monitoring: Computer assistance by the use of dedicated programs may improve dose management and TTR. Although programs differ, they typically calculate whether a dose adjustment is necessary from a user-defined table of trend rules for each therapeutic range. If dose adjustment is required, the current INR is compared with the target INR, and a proprietary equation calculates the new dose. The time to the next test is also set by the program using a set of variables comparing the current INR, the interval from the last test, the number of previous changes, and the number of previous INR values within the target range.

A number of early studies ${ }^{218-220}$ evaluated computer programs to improve warfarin dosing. The first randomized study in $1993^{221}$ showed that three contemporary computer programs all performed as well as the experienced medical staff of an anticoagulation management service in achieving a target INR of 2.0 to 3.0, but the computer achieved significantly better control when more intensive therapy was required (ie, INR, 3.0-4.5). In another randomized study ${ }^{222}$ of 101 patients receiving long-term anticoagulation, computerized warfarin dose adjustment proved comparable to manual regulation in terms of the percentage of INR values maintained within the therapeutic range but required 50\% fewer changes of dose. The first multicenter randomized trial, in 285 patients, of one computerized dosage program in $1998^{223}$ showed a $22 \%$ overall improvement of control with the program compared with the performance of experienced medical staff. The computer program gave significantly better overall INR control across all target INR ranges. A slight improvement in TTR was also obtained by Italian investigators ${ }^{224}$ using a different management program in $>1,200$ randomized patients from five centers. A total of $71.2 \%$ of patients were in range with computer dosing and $68.2 \%$ were in range by manual dosing during the maintenance phase. ${ }^{224}$ In both of these studies, the computer's improved results were probably due, in part, to a reduced propensity to reduce warfarin doses when the INR was at the upper limit of the "desired" range.

More recently, the European Concerted Action on Anticoagulation completed the first international, multicenter, randomized, controlled trial aimed to compare the safety and effectiveness of computerassisted dosing of VKAs, using two computer programs in different centers (DAWN AC; 4S Information Sys, and PARMA; Instrumentation Laboratories), with conventional manual dosing. ${ }^{225}$ A total of 13,052 patients treated with a VKA were followed for a total of 18,617 patient-years. Overall, there was a trend toward fewer clinical events with computerassisted dosage. There was also a statistically significant reduction in clinical events in the subgroup treated for VTE. A subsequent cost-effectiveness analysis found computer-assisted dosing cheaper than manual dosing. ${ }^{226}$

The results of two small clinical trials suggest that computerized dose management can also be at least as effective as manual dosing by trained anticoagulation physicians or nurses during the initiation of anticoagulation therapy. ${ }^{224,227}$ However, some computerized programs are unable to manage dosing during the induction phase.

1.7.3 Point of Care INR Testing: Point of care (POC) monitors measure a thromboplastin-mediated clotting time using a fingerstick sample of capillary whole blood or un-anticoagulated venous whole blood. ${ }^{228}$ The result is then converted to a plasma PT equivalent by a microprocessor and is expressed as a PT and/or INR. Each manufacturer typically establishes the conversion formula by simultaneously comparing fingerstick or venous whole blood results with an established laboratory method and reagent that is traceable to the international reference thromboplastin. 
Numerous studies ${ }^{229-248}$ have reported on the accuracy and precision of these instruments and on the ability of patients, both adults and children, to obtain an INR and to use that INR to guide their anticoagulant therapy. However, limitations to the accuracy and precision of POC INR monitors have been documented. Problems identified with POC instruments include greater differences compared with a standard plasmabased methodology as INRs increase above the therapeutic range, ${ }^{242,243}$ incorrect calibration of the ISI of the POC instruments, ${ }^{244}$ the inability to calculate a mean normal PT, ${ }^{249}$ and instrument-specific inaccuracies of the INR in patients with antiphospholipid antibodies. ${ }^{250}$ In a recent systematic review of the literature, Gialamas et al ${ }^{251}$ concluded that there is still no robust evidence that the use of POC instruments in general practice improves health outcomes or is cost-effective compared with UC and that analytical quality is comparable to laboratory testing. A major problem of comparative studies is the fact that there is a similar lack of correlation of INR results when anticoagulated plasmas are simultaneously compared using different instrument/thromboplastin combinations. ${ }^{129-135}$ These differences may be clinically important in that they may lead to different dosing decisions. ${ }^{128-134}$ Kaatz et al ${ }^{252}$ compared two POC monitors and four clinical laboratories against a secondary reference thromboplastin preparation. They found that laboratories using a more sensitive thromboplastin showed close agreement with the standard, whereas laboratories using an insensitive thromboplastin showed poor agreement. The two POC monitors fell between these two extremes.

Steps are still needed to ensure the conformity of POC PT monitors to the WHO INR PT standardization scheme, but the WHO ISI calibration procedure is not practicable using the monitors. Simpler procedures for ISI calibration of POC monitors have recently been evaluated in a number of multicenter sites by the European Concerted Action on Anticoagulation and the UK National External Quality Assessment Schemes. By using lyophilized plasma calibrants with independently certified INRs, Poller and colleagues ${ }^{253-255}$ have shown that verification or recalibration of the ISI of the instrument is possible. However, to obtain reliable ISI values for the two instruments tested they had to develop different ISI calibration methods. It is likely, therefore, that different types of POC monitor systems will require different ISI calibration methods. In a study of proficiency testing of three POC monitors over 6 years in $>10$ centers, Kitchen et al ${ }^{256}$ found in each survey that INR results in $10 \%$ to $11 \%$ of centers were $>15 \%$ different from results in other centers using the same monitors. This compared with a $12 \%$ difference for hospitals using conventional INR techniques. The
European Concerted Action on Anticoagulation has recently tested 523 Coaguchek monitors at nine clinics and found that $20.3 \%$ of the monitors showed significant deviations. ${ }^{257}$

PST or PSM using a POC instrument represents another model of care with the potential for improved outcomes as well as greater convenience.258 Several systematic reviews have shown improvements in the quality of anticoagulation control (TTR) and/or incidence of adverse events with PST and/or PSM.259-261 Heneghan et al ${ }^{261}$ pooled estimates from 14 randomized trials of PST showing a significant reduction in thromboembolic events (OR, 0.45; 95\% CI, 0.30-0.68), all-cause mortality (OR, 0.61; 95\% CI, 0.38-0.98), and major hemorrhage (OR, 0.65; 95\% CI, 0.42-0.99) vs the comparator. For PST and PSM combined, there were significant reductions in thromboembolic events (OR, 0.27; 95\% CI, 0.12-0.59) and death (OR, $0.37 ; 95 \%$ CI, 0.16-0.85) but not major hemorrhage (OR, 0.93; 95\% CI, 0.42-2.05). In a recent randomized study from France comparing monthly laboratory monitoring with weekly self testing and monitoring in patients with prosthetic heart valves, self monitoring improved INR stability and reduced the incidence of bleeding, although the study was underpowered to show a true difference in clinical outcomes. ${ }^{262}$ Another recent randomized study from Germany compared INR monitoring by the primary care physician with self management in patients with prosthetic heart valves and found greater INR stability and a lower incidence of thromboembolic events in the group of self-managed patients but no differences in bleeding events. ${ }^{263}$

PST and PSM require special patient training to implement. ${ }^{264,265}$ This mode of therapy may not be suitable for all patients and may not be practical or cost-effective in certain settings. All participants in PST/PSM programs should participate in a recognized external quality assessment program.

\subsection{Optimal Intensity Ranges}

The optimal target range for the INR is not the same for all indications. In general, because bleeding is closely related to the intensity of anticoagulation, ${ }^{164,266-268}$ there has been interest in establishing the lowest effective therapeutic range for each indication. ${ }^{268-276}$

Investigators have used various methodological approaches to establish the most appropriate range for different indications. These are as follows: (1) randomized trials in which patients are assigned to one of two different target ranges ${ }^{270-274} ;(2)$ indirect comparisons, in which outcomes are compared between separate randomized trials of VKA therapy that applied different target ranges of INR, and the control 
patients received no therapy or another antithrom-

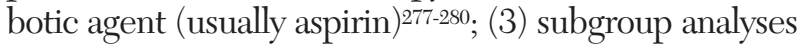
of observational studies (including within treatment groups of randomized trials) relating the observed INR or time spent in an INR range at the time of the outcome to either a bleeding event or thromboembolic event ${ }^{164,207,208,267,266,281}$; and (4) case-control studies in which the INR levels at the time of an event are recorded and compared with INR levels in appropriately selected control subjects. ${ }^{174}$

When moderate-intensity INR (approximately 2.0-3.0) was compared with higher-intensity adjusteddose oral anticoagulation, ${ }^{269,272-274,278-280}$ the moderate treatment intensity was shown to reduce the risk of clinically important bleeding without reducing efficacy. Conversely, a lower treatment intensity (eg, INR range 1.5-2.0) appears to be less effective than moderate-intensity therapy. For example, a randomized trial demonstrated that an INR of $<2.0$ (INR target, 1.5-2.0 $)^{270}$ reduced the recurrence of venous thrombosis after an initial 3 to 6 months of standard treatment when compared with placebo. A subsequent clinical trial, ${ }^{271}$ however, found that maintaining an INR intensity of 2.0 to 3.0 in the same clinical setting was more effective than a lower intensity of 1.5 to 2.0 and was not associated with a greater risk of bleeding. Likewise, in patients with $\mathrm{AF}$, a randomized trial ${ }^{282}$ reported that adjusted-dose warfarin therapy (INR, 2.0-3.0) was more effective than the combination of fixed-dose warfarin $(3 \mathrm{mg} / \mathrm{d})$ and aspirin; other studies showed that the efficacy of oral anticoagulant agents is reduced when the INR falls to $<2.0 .{ }^{174,207,208,283-285}$ Warfarin targeted to an INR of $<2.0$ has also been shown to be ineffective for preventing failure of dialysis access grafts. ${ }^{286}$

The use of fixed minidose warfarin ( $1 \mathrm{mg}$ daily) has been evaluated in a number of clinical settings. A number of these studies reported that fixed minidose warfarin is ineffective when compared with dose-adjusted warfarin, in particular for the prevention of stroke in patients with $\mathrm{AF}$ and for the prevention of thrombosis of central venous catheters. ${ }^{287-290}$ There is currently no evidence to support the use of fixed mini-doses of either acenocoumarol or phenprocoumon in any setting. However, the data obtained with fixed minidoses of warfarin cannot be extrapolated to other VKAs because of their different half-lives and the likelihood they would have different pharmacodynamic effects at similar doses. The optimal target range for each indication is discussed specifically in other articles in this supplement pertaining to each indication.

\subsection{Significance of Nontherapeutic INRs}

Fluctuations in INR may occur because of any one or more of the following conditions: inaccuracy in
INR testing, changes in vitamin $\mathrm{K}$ intake, changes in the absorption of vitamin K or VKAs, changes in the metabolism of VKAs, changes in vitamin K-dependent coagulation factor synthesis or metabolism, other effects of concomitant drug use, or patient noncompliance. A number of studies have shown that adverse event rates rise sharply as the INR moves above or below the target INR interval. ${ }^{163,164,174}$ A recent retrospective analysis of $>3,000$ patients with $\mathrm{AF}$ found that the one-third with the poorest INR control (48\% of time in range) had twice the rate of stroke, myocardial infarction, major bleeding, and death as did the one-third with the best INR control (83\% of time in range). 163

However, the risk of adverse events associated with a single INR outside the therapeutic range is probably low. For example, although excessively elevated INR values are clearly associated with an increased risk of bleeding, in particular for INR values of $>5.0,{ }^{164,169,170,268}$ data from a large registry of warfarin-treated patients suggest that the short-term risk for major bleeding is low for someone with a single INR value between 5.0 and $9.0\left(0.96 \%\right.$ at 1 month). ${ }^{291}$ Similarly, in a retrospective, matched cohort study of 2,597 patients on warfarin therapy, the risk of thromboembolic events at 3 months in patients with stable INRs who experienced a single significant subtherapeutic INR value was low $(0.4 \%)$ and was not significantly different from the risk observed in patients with persistently stable INRs. ${ }^{292}$ Similar rates were reported in a population of 294 patients with mechanical heart valves experiencing a single subtherapeutic INR and in a large cohort of patients who required short-term discontinuation of warfarin to undergo minor outpatient interventions. ${ }^{293,294}$

Before executing a plan for managing an episode of altered anticoagulation effect, important factors relating to the collection and processing of the blood sample must be taken into consideration. For example, a spuriously elevated INR should be suspected when a patient with previously stable INR control presents with a very high INR result in the absence of any explanation for the loss of INR control. Spurious results might also be suspected when abnormalities were encountered during sample collection (eg, the phlebotomist had a difficult time obtaining a sample or combined the contents of two collection tubes, and so forth). ${ }^{295}$ Recommendations for the management of patients whose INR is outside the therapeutic range are provided in Holbrook et al. ${ }^{86}$

\subsection{Adverse Events}

1.10.1 Bleeding Events: The rate of hemorrhagic events must be interpreted in the context of the clinical characteristics of the group studied. Factors that 
influence the rate of bleeding include the following: the target INR range; whether patients are mostly new to therapy or have long-term experience with therapy; whether an INR or PT is used to manage therapy; the indication for anticoagulation; the type of VKA; patient-specific risk factors, including concomitant antiplatelet therapy; and the quality of dose management. It is also not appropriate to extrapolate the rates of adverse events from randomized controlled trials to everyday practice, because high-risk patients may be excluded from clinical trials, and monitoring and management of anticoagulation are often better coordinated in clinical trials than in clinical practice.

When bleeding occurs, especially from the GI or urinary tract, the presence of an underlying occult lesion should always be considered. This is important because the patient factor that most consistently predicts major bleeding is a history of other bleeding, particularly from the GI tract. ${ }^{207}$ A number of descriptive studies ${ }^{296-298}$ have reported on the probability of finding occult lesions. Coon and Willis ${ }^{296}$ identified occult lesions that were responsible for bleeding in $11 \%$ of 292 patients with hemorrhage. Jaffin et al ${ }^{297}$ found a $12 \%$ prevalence of positive stool occult blood test results in 175 patients receiving warfarin or heparin compared with $3 \%$ in 74 control subjects. There was no difference between the mean PT or activated partial thromboplastin time (aPTT) in patients with positive and negative test results. Among the patients with positive stool occult blood test results, 15 of 16 patients had a lesion that had not been previously suspected, and four patients had neoplastic disease. Landefeld et al ${ }^{266}$ found that 14 of 41 patients with GI bleeding had important remediable lesions, of which two were malignant. To perform endoscopy in these patients is an important choice, because endoscopy has been shown in prospective studies to identify the bleeding source in $>50 \%$ of patients managed with anticoagulant therapy who present with upper GI tract bleeding and because endoscopic therapy for nonvariceal upper GI bleeding achieves hemostasis in $>90 \%$ of patients. ${ }^{299}$ This limited information supports the need to investigate patients with occult GI bleeding, as it may herald the presence of an underlying malignancy or other lesion that is frequently rectifiable.

In a 2-year prospective study in which enrolled patients had monthly urinalysis, Culclasure et al ${ }^{300}$ found microscopic hematuria in $3.2 \%$ of patients receiving oral anticoagulation compared with $4.8 \%$ in the control group not receiving anticoagulant therapy. There was no difference in the rate of hematuria with therapeutic or high INRs. Following a second episode of hematuria, 43 patients (32 receiving anticoagulant therapy, 11 control patients) were investigated.
Of these patients, 27 receiving anticoagulant therapy (84\%) and eight control patients $(73 \%)$ were found to have significant underlying disease, with three cancers found in the combined group (7\%). Other case series $^{301,302}$ have reported a higher likelihood of underlying lesions in patients who develop hematuria while receiving anticoagulant therapy.

1.10.2 Factors Predictive of Bleeding Events: The most important factor influencing the risk of bleeding is the intensity of anticoagulant therapy. $162,164,169,170,174$, 208,266-269,272-275,283-285 The likelihood of bleeding has been reported to rise steeply as the INR increases above 5.0. ${ }^{164,169,170,268}$ The optimal target range for each indication and the lowest effective range are discussed specifically in other articles in this supplement pertaining to each indication.

Several patient characteristics are associated with higher odds of bleeding during anticoagulation therapy. The patient factor most consistently predictive of major bleeding is a history of bleeding (especially GI bleeding). 162,208,273 Other factors associated with a higher risk of bleeding include advanced age; the presence of a serious comorbid condition, such as cancer, renal insufficiency, liver disease, arterial hypertension, and prior stroke; alcohol abuse; and the use of concomitant therapies, in particular antiplatelet drugs. ${ }^{162,203,204,207,303}$

A number of prediction models of bleeding risk have been proposed. Dahri and Loewen ${ }^{304}$ performed a qualitative review of the published clinical prediction rules that estimated bleeding risk in patients starting on warfarin. The authors found seven studies, of which four presented distinct clinical prediction rules. Because none of these scores exhibited sufficient predictive accuracy or evaluated the impact of their use on patient outcomes, the authors concluded that no existing clinical prediction rules can be recommended for widespread use in practice at present. One prediction model that was prospectively validated in different outpatient populations identified four independent factors associated with an increased risk of bleeding. These factors were age $>65$ years, history of gastrointestinal bleeding, history of stroke, and at least one of the following variables: myocardial infarction, hematocrit $<30 \%$, creatinine $>1.5 \mathrm{mg} / \mathrm{dL}$, and diabetes. ${ }^{203}$ Another model, derived using registry data from patients with AF, identified prior bleeding, hepatic or renal disease, ethanol abuse, malignancy, age $>75$ years, reduced platelet count or function, hypertension (uncontrolled), anemia, genetic factors (CYP2C9 polymorphism), an excessive risk of falls, and a history of stroke as risk factors for bleeding. ${ }^{305}$

The impact of age on bleeding risk remains controversial, with older reports finding risk increasing with age, ${ }^{162,163,181,267,306,307}$ whereas newer studies have failed 
to find this association. ${ }^{170,205,207,268,282,308-310}$ The discrepancy may be partly explained by the wide range in the mean age of the patients enrolled in the various studies, the relative lack of representation in most studies of patients $>80$ years of age, and the selection and survivorship biases in noninception cohort studies. When investigators attempt to separate the effect of age from comorbid conditions associated with age, some have concluded that age in and of itself is not a major independent risk factor, ${ }^{112,267,311}$ whereas others have found it to be an independent risk factor ${ }^{266,268}$ even after controlling for the intensity of the anticoagulant effect. Some have suggested that older patients may have a lower risk of bleeding when managed by AMS. 312,313 Even if the overall risk of bleeding is not increased in the elderly, it is clear that the risk of intracranial hemorrhage increases with age. ${ }^{267,268,314,315}$

Warfarin is frequently used concurrently with other antithrombotic agents. A meta-analysis assessing clinical studies comparing warfarin alone and warfarin in association with aspirin found that the combination increased the risk of bleeding by almost one-half compared with warfarin alone (OR, 1.43; 95\% CI, 1.00-2.02). ${ }^{316}$ A combined analysis of the SPORTIF (Stroke Prevention Using an Oral Thrombin Inhibitor in Atrial Fibrillation) III and V trials involving 481 patients who received aspirin and warfarin compared with 3,172 patients who received warfarin alone demonstrated a significant $1.6 \%$ per year increase in major bleeding. ${ }^{317}$ Although there are no randomized controlled trials that have compared bleeding rates in patients receiving "triple therapy" (usually warfarin, aspirin, and clopidogrel) with either warfarin alone or with a "dual therapy," a systematic review identified 12 reports involving 3,413 patients treated with oral anticoagulants who underwent percutaneous coronary intervention with stent insertion and subsequently received the combination of aspirin, clopidogrel, and warfarin. The rates of major bleeding in patients receiving triple therapy ranged from $0 \%$ to $21 \%$ (mean $7.4 \%$ ) during up to 21 months of follow-up and $0 \%$ to $5.9 \%$ (mean $2.6 \%$ ) during 30 days of follow-up. ${ }^{318}$ In a Danish nationwide registry of patients with $\mathrm{AF}$, all combinations of warfarin, aspirin, and clopidogrel were associated with an increased risk of nonfatal and fatal bleeding, whereas dual or triple therapy carried a more than threefold higher bleeding risk than warfarin alone. ${ }^{319}$

1.10.3 Reversal Strategies: Strategies to reverse the effect of VKAs may be needed in patients who require urgent invasive procedures, in asymptomatic patients presenting with excessively elevated INR values, and in bleeding patients. Therapeutic options include interruption of VKA treatment as well as the administration of vitamin $\mathrm{K}$ (usually vitamin $\mathrm{K} 1$, phytonadi- one) and blood derivatives such as fresh frozen plasma and prothrombin complex concentrates and recombinant activated factor VII. ${ }^{303,320}$

Interruption of VKAs may be sufficient in patients who need an elective invasive procedure or in asymptomatic patients with an elevated INR value and a low risk for bleeding. In this latter case, it must be noted that it takes approximately 2.5 days for an INR between 6.0 and 10.0 to decline to $<4.0 .^{321}$ Because acenocoumarol has a much shorter half-life than warfarin, the time required for an effective decline will be less and is probably no more than 1 day for most patients. ${ }^{322,323}$ Conversely, the longer half-life of phenprocoumon will probably result in a much slower decline. Finally, the half-life of fluindione is similar to that of warfarin, and thus a similar decline should be expected.

Phytonadione (vitamin $\mathrm{K}_{1}$, a form of vitamin $\mathrm{K}$ derived from plants) has been used in clinical trials that assessed the usefulness of oral vitamin $\mathrm{K}$ for the treatment of warfarin-associated coagulopathy. ${ }^{324}$ Vitamin $\mathrm{K}_{2}$ (menaquinone, which is synthesized by bacteria) and vitamin $\mathrm{K}_{3}$ (menadione) are not widely available and have not been well studied in clinical trials. ${ }^{324}$ Low doses of phytonadione can be administered orally in combination with warfarin interruption in patients not requiring urgent reversal. When oral phytonadione is administered in conjunction with temporary interruption of warfarin therapy, approximately 1.4 days are required for an INR between 6 and 10 to decline to $<4.0 .^{321}$ When administered intravenously, low doses of phytonadione produce similar reductions as oral phytonadione in the INR value at $24 \mathrm{~h}$, whereas subcutaneous phytonadione appears to be less effective than low-dose oral phytonadione. ${ }^{325,326}$ When administered at higher doses for the management of the bleeding patient, intravenously administered phytonadione works more rapidly than either oral or subcutaneous vitamin $\mathrm{K}_{1}{ }^{325,327,328}$ Reduction of the INR begins within $2 \mathrm{~h}$, and a correction to within the normal range is generally achieved within $24 \mathrm{~h}$ if hepatic function is normal and if a sufficiently large dose is given. ${ }^{324}$ At $24 \mathrm{~h}, 5$ $\mathrm{mg}$ of oral and $1 \mathrm{mg}$ or IV vitamin $\mathrm{K}_{1}$ produce similar effects on the INR. ${ }^{325}$ IV phytonadione may cause anaphylactoid reactions. Although frequently reported, and likely more common in patients who receive large IV doses administered rapidly, the true frequency of this complication is about three per 10,000 doses administered, and it may be more likely to occur if formulations containing polyethoxylated castor oil are used to maintain the vitamin $\mathrm{K}$ in solution. ${ }^{329}$ To minimize the risk of anaphylactoid reactions, vitamin $K_{1}$ should be mixed in a minimum of $50 \mathrm{~mL}$ of intravenous fluid and administered, using an infusion pump, over a minimum of $20 \mathrm{~min}$. 
Fresh frozen plasma remains the most widely used coagulation factor replacement product for urgent reversal of coumarin anticoagulation. ${ }^{320}$ Plasma may be a potential carrier of infective agents, and its use is associated with an increased risk of volume overload. Furthermore, it requires a cross-match if group-specific plasma is to be used, and it takes a prolonged period of time to thaw and administer. Given the long halflife anticoagulant effect of warfarin and the short half-life of infused coagulation factor concentrates, phytonadione must also be given to restore the adequate endogenous production of VKA-sensitive anticoagulant proteins. Urticaria occurs frequently with plasma transfusion; anaphylaxis is less common, occurring in about one in 20,000 transfusion episodes. ${ }^{330}$ Transfusion-related acute lung injury remains the most feared complication after transfusion and is estimated to occur in about one in 5,000 plasmacontaining transfusions. ${ }^{331}$

Nonactivated prothrombin complex concentrates (PCC) are probably more effective than plasma in correcting INR. PCCs do not require a cross-match, are virally inactivated, do not pose a risk of volume overload, and can be infused in 15 to $30 \mathrm{~min}$. PCC may be classified as three-factor products (with adequate levels of factors II, IX, X, and low factor VII levels) and four-factor products, which contain adequate levels of factors II, VII, IX, and X as well as protein C and S. ${ }^{319,331}$ Current PCCs are more or less devoid of activated clotting factors and are supplemented with heparin and antithrombin to minimize the risk of thrombosis. ${ }^{332}$ Four-factor PCCs are currently not available in some countries (eg, United States).

In patients with life-threatening bleeding, recombinant activated factor VII has been used to control bleeding. ${ }^{320}$ Recombinant activated factor VII is able to generate a consistent thrombin burst through both tissue factor-dependent and tissue factor-independent mechanisms and is able to trigger thrombin generation even in the presence of significant platelet dysfunction. Evidence supporting its use in VKA-associated bleeding is currently limited, and its use cannot be recommended except in the setting of life-threatening bleeding when more effective agents are not available. ${ }^{333}$ As would be expected based on its potent procoagulant effect, this agent may cause thrombosis. Recommendations to guide the use of these strategies are provided in Holbrook et al. ${ }^{86}$

\subsubsection{Nonhemorrhagic Adverse Events: Other} than hemorrhage, the most important side effects of warfarin are acute thrombotic complications, such as skin necrosis and limb gangrene. These uncommon complications are usually observed on the third to eighth day of therapy ${ }^{334,335}$ and are caused by extensive thrombosis of the venules and capillaries within the subcutaneous fat (in the case of skin necrosis) and massive outflow obstruction of the venous circulation of the limb (in the case of limb gangrene). The pathogenesis of these complications and the reason for the localization of the lesions are not well understood. An association between warfarin-induced skin necrosis and protein $\mathrm{C}$ deficiency ${ }^{336-339}$ and, less commonly, protein $\mathrm{S}$ deficiency ${ }^{339}$ has been reported, but this complication also occurs in nondeficient individuals. A pathogenic role for protein $\mathrm{C}$ deficiency is supported by the similarity of the lesions to those seen in neonatal purpura fulminans, which complicates homozygous protein $\mathrm{C}$ deficiency. A variant of this syndrome also attributed to a severe warfarin-induced depletion of protein $\mathrm{C}$ is the occurrence of venous limb gangrene during warfarin treatment of cancerassociated DVT ${ }^{340}$ and in some patients with heparininduced thrombocytopenia started on warfarin after withdrawal of heparin. ${ }^{341,342}$ The management of patients with warfarin-induced skin necrosis who require lifelong anticoagulant therapy is problematic. Therapy with warfarin is considered to be contraindicated, and long-term heparin therapy is inconvenient and is associated with osteoporosis. A reasonable approach in such patients is to restart warfarin therapy at a low dose (eg, $2 \mathrm{mg}$ ), under the coverage of therapeutic doses of parenteral anticoagulants, and to gradually increase the warfarin dose over 1 or more weeks. This approach should avoid an abrupt fall in protein $\mathrm{C}$ levels before there is a reduction in the levels of factors II, IX, and X, and it has been reported to not be associated with the recurrence of skin necrosis in a number of case reports. ${ }^{337,338,342}$

The purple toe syndrome may very rarely occur in association with the initiation of VKA treatment. It is a nonhemorrhagic, cutaneous complication due to cholesterol emboli that usually develops 3 to 8 weeks after the start of warfarin therapy and is characterized by the sudden appearance of bilateral, painful, purple lesions on the toes and sides of the feet that blanch with pressure. ${ }^{343,344}$

The VKAs also interfere with the carboxylation of Gla proteins that are synthesized in bone. ${ }^{345-348}$ Although these effects contribute to fetal bone abnormalities when mothers are treated with a VKA during pregnancy, ${ }^{349,350}$ it is unclear how they might affect children. There are two uncontrolled cohort studies that describe reduced bone density in children on warfarin for $>1$ year, but the role of the underlying disorders in reducing bone density remains unclear. ${ }^{351}$

Finally, by preventing the activation of Gla proteins and growth arrest-specific gene 6 (Gas-6), VKAs may also induce vascular calcification. ${ }^{352}$ However, this relationship in humans is conjectural and is based on case reports. ${ }^{352}$ 


\subsection{DiRECT THROMBIN INHIBITORS: DABIGATRAN ETEXILATE}

Dabigatran is a selective, reversible, direct thrombin inhibitor given as dabigatran etexilate, an orally absorbable prodrug, since dabigatran itself is a strongly polar molecule that is not absorbed from the gut. Phase 3 clinical studies reported to date have evaluated the use of dabigatran etexilate for the prevention of VTE after elective total knee or hip arthroplasty, for therapy of VTE, and to prevent stroke or systemic embolism in nonvalvular AF. The drug is approved in many countries for the prevention of VTE in patients undergoing total hip or knee replacement surgery and in the United States and Canada for the prevention of stroke or systemic embolism in nonvalvular AF. Dosing schedules are $150 \mathrm{mg}$ and $220 \mathrm{mg}$ once daily when used to prevent VTE (starting with a half dose given soon after surgery) and $110 \mathrm{mg}$ and $150 \mathrm{mg}$ bid in patients with $\mathrm{AF}$, although only the latter dose was approved for this use in the United States. The $150 \mathrm{mg}$ bid dose was also used for the treatment of VTE. Dabigatran etexilate remains under evaluation for the secondary prevention of VTE and in the management of acute coronary syndromes. Melagatran (given as its prodrug Ximelagatran) was the first of the orally available direct thrombin inhibitors to be clinically evaluated and was effective for both the prevention and treatment of VTE and in AF but caused unacceptable liver toxicity. ${ }^{353-355}$

\subsection{Pharmacology}

Direct thrombin inhibitors, such as dabigatran, the hirudins, and argatroban, do not require a cofactor, which differentiates them from the indirect coagulation inhibitors like the heparins, other glycosaminoglycans, and the synthetic pentasaccharide that must form a complex with plasma antithrombin before they can accelerate inhibition of thrombin and/or factor $\mathrm{Xa} .{ }^{356}$ Clot-bound thrombin is relatively protected from heparin-like anticoagulants in vitro but is freely accessible to direct thrombin inhibitors. ${ }^{119}$

Dabigatran (initially referred to as BIBR 953) is a small synthetic molecule of $471.5 \mathrm{~d}$ with strongly basic functional groups that is a concentration dependent, competitive, highly selective and reversible direct thrombin inhibitor with a $\mathrm{Ki}$ of $4.5 \pm 0.2 \mu \mathrm{M}$ (which is comparable with that of melagatran). 357,358 The inhibitor prevents access to the active site of thrombin by forming a salt bridge between its amidine group and Asp 189 and through hydrophobic interactions. ${ }^{357}$ Like melagatran and argatroban, dabigatran is a univalent inhibitor that interacts with the active site of thrombin alone, whereas hirudin, lepirudin, and desirudin also bind to a substrate recogni- tion site (exosite 1). ${ }^{356}$ Because the highly polar and lipophobic dabigatran molecule is not absorbed from the gut, its oral availability required the synthesis of an absorbable prodrug, dabigatran etexilate (BIBR $1048 ; 627.7$ d). ${ }^{357}$ On absorption, this esterified, hexylated, and more lipophilic prodrug is rapidly converted to dabigatran (the mechanism is ester cleavage catalyzed by serine esterase enzymes, via two intermediates-BIBR 1087E and BIBR 951 CL). ${ }^{357,359}$ As would be expected from a direct thrombin inhibitor, dabigatran prolongs the thrombin clotting time (TCT), PT, aPTT, and ecarin clotting time (ECT) of plasma from humans, rats, rabbits, dogs, and rhesus monkeys and also inhibits thrombin generation in human plasma. ${ }^{358}$ The ECT has been a preferred measure of anticoagulant effect for r-hirudin and other direct thrombin inhibitors; ecarin is a metalloprotease enzyme obtained from venom of the saw-scaled viper (Echis carinatus) that generates meizothrombin from prothrombin. ${ }^{360,361}$

Dabigatran prevents thrombin-induced platelet aggregation but not platelet aggregation by arachidonic acid, collagen, or adenosine diphosphate. ${ }^{358}$ Administration of oral dabigatran etexilate or IV dabigatran causes concentration-dependent reductions of thrombosis provoked in rats and rabbits by venous stasis plus tissue factor infusion or venous stasis plus endothelial damage. Thrombus inhibition peaks within 30 to $60 \mathrm{~min}$ of an oral dose, then persists for 2 to $3 \mathrm{~h}$ in rats and about $7 \mathrm{~h}$ in rabbits, and correlates well with prolongation of the aPTT. ${ }^{362,363}$ In rats, the intravenous dabigatran dose required to prolong the tail bleeding time is 5 to 10 times greater than the maximally effective antithrombotic dose of $0.1 \mathrm{mg} / \mathrm{kg}^{3}{ }^{363}$

\subsection{Pharmacokinetics and Pharmacodynamics}

Dabigatran etexilate is now formulated as a capsule containing multiple small pellets, each of which is composed of drug coated on a tartaric acid core to create an acid microenvironment that favors drug dissolution and preserves gut absorption even when the gastric $\mathrm{pH}$ is high (solubility is best at a low $\mathrm{pH}$ ). ${ }^{364}$ Systemic bioavailability of oral dabigatran etexilate has been measured at $7.2 \%$ in healthy young volunteers and estimated at $6 \%$ to $7 \%$ in healthy older subjects. ${ }^{359,364}$ Oral bioavailability of the capsules is comparable to that of tablets used in the phase 1 and early phase 2 evaluations. ${ }^{365,366}$ Plasma dabigatran concentrations peak within 2 hours after a dose of dabigatran etexilate and then decrease by $>70 \%$ during an initial 4- to 6 -h distribution phase that is followed by a much slower elimination phase. With repeated dosing, the terminal elimination half-life is 12 to $17 \mathrm{~h}$, the peak and trough concentrations are dose-proportional, and it takes 2 to 3 days to reach 
steady-state levels. The terminal half-life following a single dose is about $9 \mathrm{~h}$ in healthy volunteers. ${ }^{359,361,364}$ The summary of pharmacokinetic parameters in Table 3 was derived from Stangier. ${ }^{367}$ Rapid conversion of dabigatran etexilate to dabigatran ensures that plasma concentrations of the etexilate and two intermediate prodrugs barely reach detectable levels. ${ }^{359}$ Approximately $35 \%$ of circulating dabigatran is proteinbound, regardless of concentration. After a 5-mg dose of IV dabigatran in healthy volunteers, the distribution volume was measured at 69 to $90 \mathrm{~L}$, which exceeds the volume of body water, and plasma clearance was $149 \mathrm{~mL} / \mathrm{min} .{ }^{359}$ About $15 \%$ of available dabigatran is conjugated to form pharmacologically active but unstable glucuronides that account for about $20 \%$ of the total drug exposure. ${ }^{359}$ Eighty-five percent of the dose is excreted by renal clearance, almost all as unchanged dabigatran. ${ }^{359}$ Pharmacokinetic data from the phase 2 studies, together with population modeling, predict average steady-state peak and trough plasma dabigatran concentrations of 99 and $14 \mathrm{ng} / \mathrm{mL}$ after daily dosing with $150 \mathrm{mg}$ dabigatran etexilate, ${ }^{368}$ 183 and $37 \mathrm{ng} / \mathrm{mL}$ after $220 \mathrm{mg} / \mathrm{d}$, 369 and 184 and $90 \mathrm{ng} / \mathrm{mL}$ after $150 \mathrm{mg}$ bid (Table S3). ${ }^{369}$ Apart from dose, the systemic exposure to dabigatran is related to age and renal function. When older but apparently healthy volunteers aged 65 to 87 years received $150 \mathrm{mg}$ dabigatran etexilate bid, the steady-state area under curve (AUC) was 1.7 to 2 times greater than that observed in a previous study of younger men aged 18 to 45 years. The increase in drug exposure was explained by reduced renal clearance and was more obvious in older women (in whom the AUC was $3 \%$ to $19 \%$ above that in older men). ${ }^{364,367}$ In a separate parallel group volunteer study, the AUC $\infty$ after $150 \mathrm{mg}$ dabigatran etexilate was $1.5,3.2$, and 6.3 times higher in people with mild, moderate, or severe renal impairment (creatinine clearance of 51-80, 31-50, and $\leq 30 \mathrm{~mL} / \mathrm{min}$ ) than in healthy control subjects. The corresponding levels of peak plasma concentration (Cmax) were 109, 138, and $205 \mathrm{ng} / \mathrm{mL}$, compared with $85 \mathrm{ng} / \mathrm{mL}$ when renal function was normal. The terminal half-life was doubled to $28 \mathrm{~h}$ in severe renal failure, from $14 \mathrm{~h}$ in control subjects. ${ }^{370}$ Strong effects of renal function on drug concentrations were also demonstrated in patients having a hip or knee replacement, in whom dose-effect modeling predicted a steady-state Cmax of $100 \mathrm{ng} / \mathrm{mL}$ during bid dosing with $150 \mathrm{mg}$ if the creatinine clearance was $>90 \mathrm{~mL} / \mathrm{min}$, increasing to 140,180 , and $240 \mathrm{ng} / \mathrm{mL}$ as creatinine clearance diminished to 60 to 90,40 to 60 , and $<40 \mathrm{~mL} / \mathrm{min}^{366,367}$ Moderately severe liver dysfunction (Child-Pugh classification B) appears to have little effect on dabigatran pharmacokinetics, since peak plasma concentrations were reduced by $15 \%$ after $150 \mathrm{mg}$ dabigatran etexilate in twelve affected subjects, when compared with 12 healthy age- and sex-matched control subjects, whereas time to Cmax, the elimination half-life, AUC, distribution volume, and extent of glucuronidation remained unchanged. Effects on blood clotting test results were similar in the two study groups. ${ }^{371}$ The effects on blood coagulation tests closely mirror plasma dabigatran concentrations. Peak prolongation coincides with the Cmax, and clotting times decrease as dabigatran leaves the circulation. Unlike warfarin and other vitamin $\mathrm{K}$ inhibitors, which have long-lasting effects on the INR, the relatively short half-life of dabigatran means that time between dosing and blood sampling is a critical determinant of drug effect. Effects on the aPTT, INR, TCT, and ECT were studied in healthy volunteers and in patients having a hip or knee replacement. In the placebo-controlled volunteer study, wherein healthy men aged 18 to 45 years received one dose of 10 to $400 \mathrm{mg}$ dabigatran etexilate or eight hourly doses of 50 to $400 \mathrm{mg}$ for 6 days, the

Table 3-[Section 2.2] Pharmacokinetic Characteristics of Dabigatran Etexilate

\begin{tabular}{|c|c|c|c|c|c|}
\hline Population and Dosage & Tmax or Tmax, ss, h & $\begin{array}{c}\text { Cmax or } \\
\text { Cmax, ss, ng/mL/mg }\end{array}$ & $\begin{array}{c}\text { Cmin or } \\
\text { Cmin, ss, ng/mL/mg }\end{array}$ & $\begin{array}{c}\text { AUC } \infty \text { or } \\
\text { ss, }(\mathrm{ng} / \mathrm{h} / \mathrm{mL}) / \mathrm{mg}\end{array}$ & $t_{1 / 2}, h$ \\
\hline \multicolumn{6}{|l|}{ Healthy adults } \\
\hline od & $1.25-1.5$ & 0.89 & & 5.66 & 8.13 \\
\hline bid & $1.5-2.0$ & 1.16 & 0.36 & 7.4 & 11.3 \\
\hline tid & & 1.68 & 0.50 & 7.85 & 13.7 \\
\hline \multicolumn{6}{|l|}{ Healthy elderly } \\
\hline bid (men) & 3.0 & 1.48 & 0.52 & 10.9 & 12.1 \\
\hline bid (women) & 2.5 & 1.83 & 0.52 & 12.8 & 13.4 \\
\hline \multicolumn{6}{|l|}{ Patients } \\
\hline od & $6.0^{\mathrm{a}}$ & $0.41^{\mathrm{a}}$ & 0.13 & $6.41^{\mathrm{a}}$ & \\
\hline bid & 2.7 & 1.06 & 0.45 & 15.9 & \\
\hline
\end{tabular}

The results recorded for bid or tid dosing are at ss. Cmax, Cmin and AUC are normalized to a 1-mg dose of dabigatran etexilate. Results are mean or median. $\mathrm{AUC}=$ area under the curve; $\mathrm{AUC} \infty=\mathrm{AUC}$ from time zero to infinity; $\mathrm{Cmax}=$ peak plasma concentration; $\mathrm{Cmin}=$ minimum plasma concentration; od $=$ once daily; ss $=$ steady state; $\operatorname{tmax}=$ time to reach the peak plasma concentration; $\mathrm{t}_{1 / 2}=$ terminal half-life. $($ Modified with permission from Stangier et al. ${ }^{364}$ )

aMeasured after first oral dose of dabigatran etexilate following surgery for hip or knee replacement. 
drug was given as a dry powder dissolved in dilute tartaric acid ${ }^{361}$ and caused dose-dependent prolongations of aPTT, INR, TCT, and ECT. The coagulation test results correlated with plasma dabigatran concentration, the maximum clotting times coincided with Cmax, and effects persisted for $8 \mathrm{~h}$ or longer except for the aPTT and INR at the lowest doses. The peak clotting time ratios after 200 to $400 \mathrm{mg}$ of dabigatran etexilate were $>55$ for the TCT, $>5$ for the ECT, 1.5 to 1.9 for the INR, and 1.8 to 2.1 for the aPTT. The TCT, ECT, and INR increased in linear proportion with plasma dabigatran levels, but the aPTT concentration-response curve was curvilinear and flattened above concentrations of $200 \mathrm{ng} / \mathrm{mL}$. ${ }^{361,369}$ Plasma dabigatran levels also correlated closely with effects on the ECT and aPTT in the BISTRO trial, a safety study of dabigatran etexilate in patients having an elective hip or knee arthroplasty, wherein escalating doses of dabigatran etexilate were given for 6 days in tablet form after surgery. The doses were $12.5 \mathrm{mg}$ rising to $300 \mathrm{mg}$ bid or $150 \mathrm{mg}$ to $300 \mathrm{mg}$ once daily. 368 Bid dabigatran etexilate raised the peak aPTT and ECT ratios from 1.08 to 1.91 and from 0.96 to 5.17 as the dose increased from $12.5 \mathrm{mg}$ to $300 \mathrm{mg}$, whereas the mean trough levels of aPTT ratio rose from 1.0 to $1.65{ }^{368}$ Concentration-effect relationships were linear for the ECT but nonlinear for the aPTT, with better sensitivity and precision for the ECT. ${ }^{366} \mathrm{~A}$ nonlinear dose-effect on the aPTT was also found in patients with AF taking bid doses of up to $300 \mathrm{mg} .{ }^{372}$ Different aPTT reagents appear to have similar sensitivities to dabigatran, as does the activated clotting time. ${ }^{369}$ The likely impact of reduced renal function on blood clotting test results in patients given $150 \mathrm{mg}$ dabigatran etexilate bid was estimated through population modeling of data from the BISTRO I study. This predicted a maximum aPTT of $47.4 \mathrm{~s}$ with normal renal function, increasing to $54.2,61.9$, and $78.3 \mathrm{~s}$ in patients with mild, moderate, or severe reductions in creatinine clearance (the corresponding predictions for maximum ECT were 55.2, 77.4, 108, and $183 \mathrm{~s}$ ). ${ }^{367}$ The modeling is consistent with results when otherwise healthy volunteers with a creatinine clearance $<30 \mathrm{~mL} / \mathrm{min}$ received a 150-mg dose of dabigatran etexilate: maximum aPTT, ECT, INR, and TCT ratios were 3.45 , $3.26,4.14$, and 12.5 , compared with $1.85,1.89,1.4$, and 6.87 in healthy control subjects. ${ }^{370}$ This emphasizes the need to avoid dabigatran etexilate in patients with severe renal failure and the importance of renal function when considering the choice of dosing regimen. It remains possible that dabigatran may activate platelets in patients with $\mathrm{AF}$, as urinary excretion of 11dehydrothromboxane B2 (an end product of thromboxane A2 metabolism) was increased by roughly $20 \%$ during 12 weeks of treatment in a dose-ranging trial, and that effect was suppressed by aspirin. ${ }^{372}$

\subsection{Interactions}

2.3.1 Factors Interfering With Absorption: Absorption of dabigatran etexilate is influenced by gastric $\mathrm{pH}$ as affected by proton pump inhibitors, food, the postoperative state, and also by drugs that inhibit or induce activity of the cell efflux transporter P-glycoprotein (P-gp). Dabigatran etexilate has a low aqueous solubility that is further reduced by increased $\mathrm{pH},{ }^{373}$ as is observed in patients taking the gastric proton pump inhibitor pantoprazole. Twicedaily pretreatment with $40 \mathrm{mg}$ pantoprazole for $48 \mathrm{~h}$ in a crossover study reduced geometric mean levels of Cmax and AUC after $150 \mathrm{mg}$ dabigatran etexilate by $40 \%$ and by $32 \%$ in healthy male volunteers aged 18 to 55 years. ${ }^{365}$ Bioavailability (steady-state AUC) was also reduced by $20 \%$ to $40 \%$ in a parallel group study in which older volunteers, aged $\geq 65$ years, took $40 \mathrm{mg}$ pantoprazole with $150 \mathrm{mg}$ dabigatran etexilate bid for 6 days; pantoprazole raised the gastric $\mathrm{pH}$ from 2.2 to 5.9 , and $\mathrm{pH}$ correlated with AUC. Small corresponding changes in Cmax, ECT, and aPTT were not believed to have clinical importance. ${ }^{364}$

Taking $150 \mathrm{mg}$ dabigatran etexilate after a high-fat, high-calorie breakfast prolonged the time taken to reach Cmax from $2 \mathrm{~h}$ to $4 \mathrm{~h}$ in the crossover study described above, although Cmax and total drug exposure remained unchanged. ${ }^{365}$ Absorption of doses taken 4 to $8 \mathrm{~h}$ after a hip replacement was slowed and reduced, compared with 2 to 10 days later, such that time to reach the peak plasma concentration was delayed to $6 \mathrm{~h}$ and both Cmax and AUC were greatly diminished (Table 3); the changes were attributed to early effects of surgery on GI motility and gastric acidity. ${ }^{366}$

2.3.2 Other Drug-Drug Interactions: Important drug-drug interactions most often result from changes in drug metabolism that are due to induction or inhibition of CYP3A4 and other enzymes of the microsomal cytochrome P450 complex or from changes in drug bioavailability mediated by the adenosine diphosphate-dependent cell efflux transporter, P-glycoprotein (P-gp). ${ }^{374}$ Potential drug interactions with dabigatran etexilate have been explored in studies in which volunteers received dabigatran etexilate together with drugs known to provoke such mechanisms. Many drugs may interact through more than one pathway.

Because cytochrome P450 enzymes have almost no role in the metabolism of dabigatran and are not affected by dabigatran in vitro, this becomes an unlikely mechanism for drug-drug interactions, 359 and volunteer studies confirm the lack of a clinically important interaction with atorvastatin (a substrate for CYP3A4 and substrate/inhibitor of P-gp) and diclofenac (a substrate for CYP2C9 and uridine 
glucuronyltransferase $2 \mathrm{~b} 7$, and also a substrate and weak inhibitor of UGT1A). ${ }^{367}$ When 22 volunteers aged $43 \pm 15$ years took $80 \mathrm{mg}$ atorvastatin together with $150 \mathrm{mg}$ dabigatran bid for 4 days in an openlabel crossover study, the steady-state AUC of dabigatran was reduced by $18 \%$, whereas the Cmax and AUC of atorvastatin increased by $15 \%$ and $23 \% .375$ Cmax and AUC of dabigatran remained unchanged in a similar study of 24 volunteers aged 18 to 55 years who took one 50-mg dose of diclofenac after 4 days of bid dosing with $150 \mathrm{mg}$ dabigatran etexilate, whereas Cmax of diclofenac and its main metabolite decreased by $11 \%$ to $17 \% .376$ The changes were believed to be small and clinically unimportant.

In vitro studies find that dabigatran etexilate (but not dabigatran) is a substrate for P-glycoprotein (P-gp, MDR1) with a medium affinity when tested using the Caco-2 cell-line1, which makes it a potential target for P-gp-related drug interactions. ${ }^{377}$ The bioavailability of P-gp substrates like dabigatran may be raised or reduced through inhibition or induction of P-gp: the P-gp inhibitors include amiodarone, verapamil, ketoconazole, quinidine, and clarithromycin, whereas P-gp inducers include rifampicin and St. John's wort (Hypericum perforatum). ${ }^{377}$

In formal interaction studies with amiodarone, a first dose of $600 \mathrm{mg}$ raised the AUC and Cmax of dabigatran by about $50 \%$ and $60 \%$, an interaction that may persist for some weeks after stopping amiodarone due to the long half-life of this drug. ${ }^{373}$ The effects of verapamil depend on its dosing schedule and drug formulation. ${ }^{373}$ The first dose of an immediaterelease formulation, when given 1 hour before $150 \mathrm{mg}$ dabigatran etexilate, increased the Cmax and AUC of dabigatran by about $180 \%$ and $150 \%$, but these elevations were reduced to about $60 \%$ and $50 \%$ after repeated dosing and to about $90 \%$ and $70 \%$ when taking an extended-release formulation. The interaction became negligible (increases of $10 \%$ in Cmax and $20 \%$ for AUC) if verapamil was taken $>2 \mathrm{~h}$ after dabigatran etexilate when dabigatran absorption was essentially complete. Twice-daily coadministration of $500 \mathrm{mg}$ clarithromycin increased the AUC and Cmax of dabigatran by about $19 \%$ and $15 \%$, respectively.

The strong P-glycoprotein inhibitors quinidine and ketoconazole are contraindicated when taking dabigatran etexilate because they markedly increase exposure to dabigatran. ${ }^{373}$ The approved product information advises caution when considering coadministration of strong P-gp inducers like rifampicin or St. John's wort, which may significantly decrease the Cmax and AUC. ${ }^{373}$

The P-gp substrate digoxin is used to probe P-gp mediated drug-drug interactions. After 4 days of dosing with once-daily digoxin plus bid $150 \mathrm{mg}$ dab- igatran etexilate, there was little effect on the pharmacokinetics of either drug in a three-way crossover study of 23 healthy volunteers aged 18 to 65 years. ${ }^{378}$

Drug interactions that may change the Cmax and AUC of dabigatran have not been correlated with clinical outcomes. Very large increases of Cmax and AUC, like those of quinidine and systemic ketoconazole, are likely to raise the bleeding risk. Moderate increases, like those provoked by amiodarone or verapamil, may become important if combined with old age or reduced renal clearance. The concern about strong P-gp inducers like rifampicin is their potential to decrease drug exposure and therefore reduce efficacy.

\subsubsection{Dabigatran Etexilate and Antiplatelet Drugs:} The added bleeding risk when platelet function inhibitors like aspirin and clopidogrel are taken during anticoagulant therapy is compounded for aspirin and other nonsteroidal antiinflammatory drugs by the increased likelihood of peptic ulceration due to interference with prostaglandin-mediated cytoprotection of the gastrointestinal mucosa. ${ }^{379}$ These mechanisms are independent from any pharmacokinetic drug-drug interactions (none was demonstrated between dabigatran etexilate and diclofenac). ${ }^{376}$ Aspirin increased the bleeding rate when added to ximelagatran in patients with $\mathrm{AF}$ and to warfarin in patients with $\mathrm{AF}$, a prosthetic heart valve, coronary artery disease, or peripheral vascular disease. .22,317,380 $^{2}$

The added effects on bleeding when aspirin is combined with dabigatran etexilate were explored in the Prevention of Embolic and Thrombotic Events in Patients With Persistent Atrial Fibrillation (PETRO) trial, a phase 2 , parallel-group, randomized, doseranging safety study in 502 patients with nonvalvular AF who also had coronary artery disease and/or one or more other risk factors for systemic embolism. The patients received 12 weeks of treatment with openlabel warfarin alone (target INR 2-3) or with blinded $50 \mathrm{mg}, 150 \mathrm{mg}$, or $300 \mathrm{mg}$ dabigatran etexilate bid plus daily aspirin ( $81 \mathrm{mg}$ or $325 \mathrm{mg}$ ) or a placebo, using a $3 \times 3$ factorial design to allocate study treatments to patient groups of unequal size. Aspirin increased the chances of major or clinically significant nonmajor bleeding in patients given a supratherapeutic dabigatran dose of $300 \mathrm{mg}$ bid, in whom the bleeding rate was $20 \%$ with an aspirin dose of $325 \mathrm{mg} / \mathrm{d}$ ( six of 30), $14.7 \%$ if the dose was $81 \mathrm{mg} / \mathrm{d}$ (five of 34), and $5.7 \%$ (six of 105) in patients given the aspirin placebo. The trends reached statistical significance when the two aspirin groups were pooled. Bleeding risk was not apparently raised when aspirin was added to 50-mg or 150-mg doses of dabigatran, but the sample size was too small to exclude clinically important effects. ${ }^{372}$ 
Low doses of aspirin $(\leq 100 \mathrm{mg} / \mathrm{d})$ were permitted in the Randomized Evaluation of Long-term Anticoagulant Therapy (RE-LY) (nonvalvular AF) and Dabigatran in the Treatment of Venous Thromboembolism (RE-MEDY) (VTE) phase 3 studies of dabigatran etexilate, which compared bid doses of $110 \mathrm{mg}$ and/or $150 \mathrm{mg}$ with warfarin, but subgroup analyses of bleeding risk are not yet available. ${ }^{381,382}$ Product information recommends against the combination of dabigatran etexilate with clopidogrel and other thienopyridines, given alone or as dual antiplatelet therapy with aspirin.

2.4 Antithrombotic Effects: A number of recent phase III clinical trials have evaluated the use of dabigatran etexilate for the prevention and treatment of VTE and to prevent systemic embolism in nonvalvular AF. A phase II study explored its dose-response in acute coronary syndromes.

The efficacy and safety of anticoagulant prophylaxis for VTE depends in any given patient population on the dose, timing, duration, and choice of drug therapy (ie, on the treatment regimen as well as the therapeutic agent). The VTE prevention trials of dabigatran etexilate have evaluated two once-daily dabigatran dosing regimens in patients having elective hip or knee arthroplasty: $150 \mathrm{mg} / \mathrm{d}$ or $220 \mathrm{mg} / \mathrm{d}$, beginning soon after surgery with a half-dose of $75 \mathrm{mg}$ or $110 \mathrm{mg}$. In addition, these two dabigatran regimens were compared with one of two standard enoxaparin prophylaxis regimens: $40 \mathrm{mg} / \mathrm{d}$ starting on the evening before surgery, or $30 \mathrm{mg}$ bid starting 12 to $24 \mathrm{~h}$ after surgery (worldwide, these are the low-molecular-weight heparin prophylaxis regimens most widely used for joint surgery; both are accepted as standard clinical practice). Two of the trials compared both of these dabigatran regimens with the $40 \mathrm{mg} / \mathrm{d}$ schedule of enoxaparin in hip or knee arthroplasty ${ }^{383,384}$ one compared both of the dabigatran regimens with $30 \mathrm{mg}$ enoxaparin bid after knee arthroplasty, ${ }^{385}$ and one compared only the $220 \mathrm{mg} / \mathrm{d}$ dabigatran etexilate regimen with enoxaparin $40 \mathrm{mg} / \mathrm{d}$ in hip arthroplasty. ${ }^{386}$

In all prophylaxis studies, the primary measure of treatment effectiveness was the rate of "total VTE" (a composite of subclinical deep leg vein thrombosis detected with routine bilateral ascending venography done at the end of treatment, confirmed symptomatic DVT or pulmonary embolism (PE), and death from any cause), an outcome that could be evaluated in $73 \%$ to $78 \%$ of patients. All recorded the presence of major bleeding, clinically relevant nonmajor bleeding, and any adjudicated bleeding.

The two dabigatran prophylaxis regimens were "noninferior" to enoxaparin $40 \mathrm{mg} / \mathrm{d}$ in hip arthroplasty and in knee arthroplasty (ie, statistically no less effective), ${ }^{383,384,387}$ but each was less effective after knee arthroplasty than $30 \mathrm{mg}$ enoxaparin bid. ${ }^{385}$ The first dose of dabigatran etexilate was given 1 to $4 \mathrm{~h}$ after surgery in the comparisons with $40 \mathrm{mg} / \mathrm{d}$ enoxaparin, but delayed until 12 to $24 \mathrm{~h}$ after operation in the comparison with bid enoxaparin. It is likely the bid enoxaparin regimen was superior in part due to the higher daily dose $(60 \mathrm{mg})$, but delaying the start of dabigatran etexilate after surgery may also have contributed to an inferior result. In a subsequent comparison, $220 \mathrm{mg} / \mathrm{d}$ dabigatran was again "noninferior" to $40 \mathrm{mg} / \mathrm{d}$ enoxaparin in preventing "total VTE" after hip arthroplasty but appeared also to be more effective in preventing "major VTE" (a composite of proximal DVT, nonfatal $\mathrm{PE}$, and death related to VTE). ${ }^{386}$ It is now standard practice to start anticoagulant treatment after VTE with a heparin (unfractionated or low molecular weight) together with a VKA, overlap the two drugs for at least 5 days, and stop the heparin only after the INR exceeds 2.0 for $>2$ consecutive days.

Dabigatran etexilate was noninferior to warfarin in a double-blind, placebo-controlled and randomized, phase III comparison in patients with deep leg vein thrombosis or PE (the RE-COVER trial) in which study treatment began with at least 5 days of an approved anticoagulant (predominantly unfractionated or low-molecular-weight heparin) plus daily warfarin placebo or warfarin. ${ }^{382}$ This study design is unlike that of other (ongoing) evaluations of new oral anticoagulants for VTE, in which the new oral anticoagulant is given alone from the initiation of therapy. The primary measure of efficacy was the incidence of symptomatic and confirmed nonfatal recurrence, or death related to VTE. Randomization in RE-COVER was substratified for a clinical presentation with symptomatic PE (31\% of the total) and subgroup analysis suggests this presentation had little or no effect on the relative efficacy of dabigatran. Masking was preserved by using coded POC machines to generate a real or sham INR. Once this exceeded 2.0 for 2 consecutive days, they received dabigatran etexilate $150 \mathrm{mg}$ or its placebo bid (ie, they continued warfarin or started dabigatran). Hence, all patients received at least 5 days of initial treatment with a heparin. However, the median total duration of parenteral therapy before starting dabigatran was 9 days in both of the RE-COVER study groups, which seems longer than the usual clinical practice. INR was within its targeted range of 2.0 to 3.0 for $60 \%$ of study time in the warfarin-treated patients.

VKAs are highly effective in preventing embolic stroke from $\mathrm{AF}$, achieving risk reductions of almost $70 \%$ when compared with placebo and about $50 \%$ when compared with aspirin ${ }^{387}$; their most feared complication is intracranial bleeding, with an added 
risk of approximately $0.2 \%$ per annum during ongoing warfarin treatment. ${ }^{388}$

Dabigatran dosing regimens of $110 \mathrm{mg}$ and $150 \mathrm{mg}$ bid were compared double-blind with open-label warfarin (target INR, 2.0-3.0) in the RE-LY trial where patients with nonvalvular AF received study treatment of at least 1 year and median of 2 years, and the primary study outcome was ischemic stroke or systemic embolism. ${ }^{381}$ On average, the INR in patients taking warfarin was within its target range of 2.0 to 3.0 for $64 \%$ of study time. Both efficacy and bleeding risk of dabigatran etexilate depended on the dose. Annual rates of ischemic stroke or embolism were $1.69 \%$ with warfarin, $1.53 \%$ with $110 \mathrm{mg}$ dabigatran bid (noninferior), and $1.11 \%$ with $150 \mathrm{mg}$ dabigatran $(P<.001$ relative to warfarin, $P=.005$ relative to $110 \mathrm{mg}$ dabigatran). The annual incidence of major bleeding was similar, with $150 \mathrm{mg}$ dabigatran and warfarin, but significantly less with $110 \mathrm{mg}$ dabigatran (see section 2.6 "Practical Issues Related to Initiation and Maintenance"). A striking result was the reduced frequency of hemorrhagic stroke with dabigatran compared with warfarin $(P<.001)$, regardless of dabigatran dose.

\subsection{Monitoring Anticoagulant Intensity}

Ex vivo effects on laboratory tests after taking dabigatran etexilate were summarized in section 2.2 "Pharmacokinetics and Pharmacodynamics." Simple widely used coagulation tests have limitations for measuring dabigatran effect: the PT and aPTT are relatively insensitive, the relation between aPTT and dabigatran concentration is nonlinear, and the usual form of TCT is oversensitive. Most promising is the ECT, which has a linear dose-response throughout the range of concentrations expected during prophylaxis or therapy; however, this test is not readily available. ${ }^{369}$

There is no evidence relevant to the possible clinical benefits from laboratory testing, since the phase 3 studies evaluated fixed doses and their reports have not examined clinical outcomes in relation to drug levels or clotting test results. The intent has been to recommend standard doses for most patients; although first principles suggest that laboratoryassisted dose adjustment of this mainly renally excreted drug could add clinical value in selected populations, as in elderly subjects with reduced renal function, 389 it seems unlikely that routine monitoring would yield any wide clinical benefit. ${ }^{390}$ Perhaps the most likely role for laboratory testing may be in treated patients who bleed or develop thrombosis, need an acute invasive procedure, or could have taken an overdose. In the setting of major bleeding, or if urgent or emergent surgery is required, a normal TCT rules out the presence of dabigatran. TCT tests are available routinely in many laboratories.

\subsection{Practical Issues Related to Initiation and Maintenance}

Unlike other new oral anticoagulants, dabigatran etexilate offers a choice between higher and lower dosing regimens, since the schedules evaluated in phase III clinical studies to date were $150 \mathrm{mg}$ and $220 \mathrm{mg}$ once daily when used to prevent postoperative VTE, $150 \mathrm{mg}$ bid for the treatment of VTE, and $110 \mathrm{mg}$ and $150 \mathrm{mg}$ bid in patients with AF.

Dose was important during long-term therapy of patients with $\mathrm{AF}$, in whom $150 \mathrm{mg}$ bid was superior to warfarin and $110 \mathrm{mg}$ bid was noninferior, whereas the rates of major bleeding were similar with $150 \mathrm{mg}$ dabigatran and warfarin but significantly less with $110 \mathrm{mg}$ dabigatran. However, only the 150-mg dose was approved in the United States, whereas in Canada both doses are approved. By contrast, there was less apparent effect of prophylactic dosing level on efficacy or the likelihood of surgical bleeding after major joint replacement, when compared with $40 \mathrm{mg} / \mathrm{d}$ enoxaparin.

Subgroup analyses of large clinical studies have provided little evidence to date for clinically important effects of age, gender, or renal function on efficacy or safety within any dosing level. Without such information, the choice between available dosing regimens must rest on individual decisions about the preferred balance between efficacy and bleeding risk and on extrapolations about that risk from pharmacokinetics of this predominantly renally excreted drug. For example, a lower dose may be preferred for elderly patients with a mild to moderate decrease of renal function. In addition, the choice will be influenced by known drug interactions (see section 2.3 "Interactions").

At this time, the only product information approved by European Medicines Agency (EMA) and other regulators is for prophylactic dosing with dabigatran etexilate. Based on clinical study results and pharmacokinetic evidence, EMA recommends a standard daily dose of $220 \mathrm{mg}$, reduced to $150 \mathrm{mg}$ if patients are aged $>75$ years, have a creatinine clearance between 30 and $50 \mathrm{~mL} / \mathrm{min}$, or need treatment with verapamil or amiodarone. Ensuring compliance with continued dosing will be a concern for dabigatran etexilate, as it is with any chronic therapy that does not require repeated measurement of drug effect.

\subsection{Adverse Events}

2.7.1 Bleeding Events: The major potential hazard from dabigatran etexilate is bleeding. After an 
elective hip or knee replacement, the risks of major bleeding were small during VTE prophylaxis with dabigatran etexilate and similar to those with prophylactic enoxaparin. The rates were $0.6 \%$ to $1.3 \%$ in patients given $150 \mathrm{mg} / \mathrm{d}$ and $0.6 \%$ to $2.0 \%$ when the dose was $220 \mathrm{mg} / \mathrm{d}$. These compared with $1.3 \%$ to $1.6 \%$ with $40 \mathrm{mg} / \mathrm{d}$ of enoxaparin and $1.4 \%$ with $30 \mathrm{mg}$ of enoxaparin given bid. About $90 \%$ of the bleeding events were related to the surgical wound.

Bleeding risk during 6 months of treatment after VTE was less with $150 \mathrm{mg}$ dabigatran bid than with warfarin (target INR, 2.0-3.0). The 6-month rate of "any bleeding" was reduced from $21.9 \%$ with warfarin to $16.1 \%$ (relative risk, $0.71 ; 95 \%$ CI, 0.59-0.85), and that of "major or clinically relevant but nonmajor" bleeding was diminished from $8.8 \%$ to $5.6 \%$ (relative risk, 0.63; 95\% CI, 0.47-0.84; $P=.002$ ). Major bleeding was infrequent (1.9\% with warfarin and $1.6 \%$ with dabigatran). ${ }^{382}$

As indicated earlier, the bleeding risk was dose dependent in the RE-LY study of patients with AF: the annual incidence of major bleeding was $3.4 \%$ in patients taking warfarin, $3.1 \%$ with $150 \mathrm{mg}$ bid dabigatran (relative risk, $0.93 ; P=.31$ ), and $2.7 \%$ if the dose was $110 \mathrm{mg}$ (relative risk, 0.80; 95\% CI, $0.69-0.93 ; P=.003$ ); the $P$ value of the difference between dabigatran dosing groups almost reached the conventional level for statistical significance $(P=.052)$. Both dabigatran regimens reduced the likelihood of intracranial bleeding by more than one-half compared with warfarin (annual incidence was $0.74 \%$ with warfarin, $0.30 \%$ with $150 \mathrm{mg}$ dabigatran $[P<.001]$ and $0.23 \%$ with $110 \mathrm{mg}$ dabigatran $[P<.001])$, whereas annual rates of major extracranial bleeding were little different between the study groups. ${ }^{381}$

Dabigatran etexilate does cause a dose-dependent increase in the incidence of major GI bleeding. This was a statistically significant effect in the RE-LY study; annual rates were $1.02 \%$ with warfarin, $1.51 \%$ with $150 \mathrm{mg}$ dabigatran $(P<.001$ compared with warfarin), and $1.12 \%$ with $110 \mathrm{mg}$ dabigatran. ${ }^{381}$ An excess risk was also reported in the RE-COVER trial, in which more patients treated with bid $150 \mathrm{mg}$ dabigatran etexilate had GI bleeding ( 53 events vs 35 with warfarin). 382

2.7.2 Nonhemorrhagic Adverse Events: Dyspepsia is a consistently reported adverse effect of dabigatran etexilate. In the RE-LY study, in which warfarin was open label, $11.8 \%$ and $11.3 \%$ of patients given $110 \mathrm{mg}$ or $150 \mathrm{mg}$ dabigatran complained of dyspepsia compared with $5.8 \%$ of patients taking warfarin. ${ }^{381}$ Fewer patients reported dyspepsia in the wholly double-blind RE-COVER trial: $2.9 \%$ with $150 \mathrm{mg}$ dabigatran bid, and $0.6 \%$ with warfarin $(P<.001) .382$
It is essential to exclude liver toxicity when evaluating new oral anticoagulants, especially because the first orally available direct thrombin inhibitor (ximelagatran) caused unacceptable hepatic toxicity that remains unexplained. About $0.5 \%$ of the patients treated with ximelagatran for $>35$ days developed laboratory evidence of severe liver injury: a greater than threefold elevation of serum alanine transaminase level plus a greater than twofold increase in bilirubin concentration, compared with upper normal limits. ${ }^{355,391}$ In one 35-day VTE prevention study, severe hepatic injury with onset after the end of drug exposure was not predicted by regular liver enzyme monitoring during therapy. ${ }^{392}$ It has been estimated that ximelagatran may have caused or contributed to fatal liver damage in about 1 in 2,000 patients. ${ }^{355}$ No dabigatran study to date has recorded a disproportionate increase of liver enzyme levels or clinically significant liver disease as an adverse effect.

In some ximelagatran studies, short- or long-term treatment was also associated with trends or a statistically significant excess of adverse events related to coronary artery disease. ${ }^{355}$ This raised concerns that other new oral anticoagulants might increase the risk from acute cardiac events. Adjudicated coronary events (unstable angina, myocardial infarction, cardiac death) were rare and evenly distributed between study groups in the orthopedic surgery studies of dabigatran etexilate and in the RE-COVER trial, but the RE-LY trial reported an excess of myocardial infarction among the patients treated with dabigatran. The relative risks, compared with warfarin, were 1.35 $(P=.07)$ with $110 \mathrm{mg}$ and $1.38(P=.048)$ with $150 \mathrm{mg}$ dabigatran (the corresponding absolute risks were $0.53 \%, 0.72 \%$, and $0.74 \%$ per annum). ${ }^{381}$ There are no ready explanations (although indirect evidence of platelet activation by dabigatran was reported from the PETRO study). ${ }^{372}$

\subsection{Reversal of Drug Effect}

There is insufficient clinical experience to firmly guide the management of major bleeding, suspected overdose, urgently needed surgery, or urgent invasive diagnostic or therapeutic procedures in patients who are taking this new drug. Pharmacokinetic modeling does, however, indicate how long it takes for drug effects to dissipate after stopping dabigatran etexilate before an elective intervention, although conclusions about the time taken before a return to normal hemostasis remain tentative pending welldocumented information about clinical outcomes.

The half-life of dabigatran suggests that drug levels and drug effects should decrease by about $50 \%$ at 12 to $18 \mathrm{~h}$ after the most recent dose, and the trough levels to $25 \%$ of their previous steady state by $24 \mathrm{~h}$ 
after stopping dabigatran etexilate, so long as creatinine clearance exceeds $50 \mathrm{~mL} / \mathrm{min}$. The level at which it is safe to undertake surgery or an invasive procedure is unknown. Moderately severe renal dysfunction (creatinine clearance of $30-50 \mathrm{~mL} / \mathrm{min}$ ) extends the half-life to about $18 \mathrm{~h}$, in which case, or if the surgical bleeding risk is critically high (as with intracranial surgery), it may be better to delay elective procedures until 2 to 4 days after stopping the drug. Measuring the TCT or aPTT should help to estimate the residual level of dabigatran. ${ }^{369}$

In addition to immediately stopping drug administration, the clinical management of major bleeding would require early volume and RBC replacement, urgent assessment for cause, and any local measures that may be required until the bleeding stops (pressure, cautery, suture, or other interventions). It is believed that maintaining an adequate diuresis could help to protect the renal excretion of dabigatran. Although product information for dabigatran etexilate mentions the use of fresh frozen plasma to help control bleeding, this seems unlikely to influence the drug effects. Thus plasma should only be administered in the setting of a documented dilutional coagulopathy.

Dabigatran has no antidote, and the management of life-threatening bleeding remains empirical. Indirect evidence from animal models and in vitro studies suggests that recombinant factor VIIa or a prothrombin complex concentrate may bypass the anticoagulant effects of high dabigatran concentrations. ${ }^{369}$ It may also be relevant that hemodialysis removed $62 \%$ of circulating dabigatran within $2 \mathrm{~h}$ and $68 \%$ within $4 \mathrm{~h}$ in an open-label study of 12 subjects with end-stage renal failure who received $50 \mathrm{mg}$ dabigatran etexilate. ${ }^{369,370}$ In vitro mixing experiments suggest that early administration of activated charcoal might reduce the absorption of dabigatran etexilate. ${ }^{369}$

\subsection{Direct FACTOR Xa InHibitors: Rivaroxaban}

Rivaroxaban is a direct factor Xa inhibitor and is currently approved in many countries, including the United States, for the prevention of VTE in patients undergoing total hip or knee replacement surgery. The drug is undergoing an extensive clinical development program in other clinical settings, including the treatment of VTE and the prevention of acute ischemic stroke in patients with AF. In phase III clinical trials, rivaroxaban was found to be more effective than the low-molecular-weight heparin enoxaparin in preventing VTE after total hip or knee replacement surgery ${ }^{393-396}$ and more effective than placebo when given to patients with previous DVT or PE after an initial 6- to 12-month course of standard oral anticoagulant treatment. The doses evaluated were $10 \mathrm{mg}$ once daily in major orthopedic surgery and $20 \mathrm{mg}$ once daily in the long-term secondary prevention of VTE.

\subsection{Pharmacology}

Rivaroxaban is a selective and competitive activesite-directed, reversible factor Xa inhibitor with selectivity for factor Xa that is $>10,000$-fold that for other trypsin-like serine proteases. ${ }^{397}$ Rivaroxaban acts through electrostatic interaction with Asp189 in the $\mathrm{S} 1$ pocket of factor $\mathrm{Xa}$. This interaction involves the chlorine substituent of the chlorothiophene moiety, which interacts with the aromatic ring of Tyr228 at the bottom of the $\mathrm{S} 1$ pocket. ${ }^{398}$ Because rivaroxaban is a nonbasic, small molecule (the molecular weight is $436 \mathrm{~g} / \mathrm{mol}$ ) it can inhibit not only free factor Xa but also prothrombinase complex and clot-associated factor Xa. ${ }^{399,400}$ This range of activities is unique to small, direct inhibitors because the factor Xa incorporated in the prothrombinase complex is protected from inhibition by antithrombin and by antithrombin-dependent anticoagulants.

Rivaroxaban is minimally soluble in organic solvents and nearly insoluble in water. ${ }^{398}$ Binding to plasma proteins, mainly albumin, ranges between $92 \%$ and 95\%.398 Rivaroxaban is metabolized via oxidative and hydrolytic pathways involving different classes of enzymes. In humans, CYP3A4 and CYP2J2 are the two enzymes responsible for its oxidative metabolism and contribute to a similar extent. ${ }^{401}$ About $66 \%$ of rivaroxaban is excreted via the kidneys (36\% unchanged), and $28 \%$ is excreted in the feces (7\% unchanged).

Inhibition of factor $\mathrm{Xa}$ activity by rivaroxaban is highly dependent on the concentration of the drug. Rivaroxaban induces prolongation of the PT, aPTT, and heparin clotting time, among other tests. ${ }^{399,402}$ On the other hand, rivaroxaban does not affect the bleeding time or platelet aggregation. ${ }^{399,403}$ Animal models demonstrated dose-dependent reduction of thrombus formation by rivaroxaban. ${ }^{399}$

\subsection{Pharmacokinetics and Pharmacodynamics}

In healthy men aged 19 to 45 years, single doses of rivaroxaban administered after a fasting period of $10 \mathrm{~h}$ produced a median inhibition of factor Xa activity that ranged from $20 \%$ with the 5 -mg dose to $61 \%$ with the maximum dose of $80 \mathrm{mg} .{ }^{404}$ No significant inhibition was observed with doses of $<5 \mathrm{mg}$. The maximum inhibition of factor Xa activity occurred between 1 and $4 \mathrm{~h}$ after drug administration, and the half-life of the biologic effect was 6 to $7 \mathrm{~h}$. Factor Xa activities did not return to normal until after $24 \mathrm{~h}$, when doses $>5 \mathrm{mg}$ were administered. ${ }^{404}$ The effect on PT prolongation had a similar profile, as did effects 
on the aPTT and HEPTEST (a low-molecular-weight heparin activity assay). Conversely, rivaroxaban had no effect on thrombin and antithrombin activity. ${ }^{404}$ Peak plasma concentration of the drug occurred at $2 \mathrm{~h}$, and the terminal half-life was between 7 and $17 \mathrm{~h}$. At doses $>10 \mathrm{mg}$, the increases in peak plasma concentration and AUC were less than dose proportional. Approximately $40 \%$ of the administered dose was excreted unchanged via the kidneys when the 1.25-mg dose was administered; this proportion decreased to $10 \%$ with the highest doses tested (ie, $60-80 \mathrm{mg}$ ). Finally, inhibition of factor Xa activity and PT prolongation correlated strongly with plasma concentrations $\left(r=0.949\right.$ and $r=0.935$, respectively). ${ }^{404}$ When multiple doses were administered at mealtime in healthy male subjects aged 20 to 45 years, the maximum inhibition of factor Xa activity was documented after approximately $3 \mathrm{~h}$ and it was dose dependent, ranging from $22 \%$ after $5 \mathrm{mg}$ to $68 \%$ after $30 \mathrm{mg}$ (Table 4). ${ }^{405}$ Inhibition was maintained for 8 to $12 \mathrm{~h}$ after $5-\mathrm{mg}$ doses and for approximately $12 \mathrm{~h}$ after doses of $10 \mathrm{mg}$ to $30 \mathrm{mg}$. Daily rivaroxaban doses did not cause a further increase in the maximum inhibition of factor Xa activity. A very similar pattern was observed with PT, aPTT, and HEPTEST prolongation, which was dose dependent for all tests, reached maximum levels after 1 to $4 \mathrm{~h}$, and was comparable after the first and last administered dose. ${ }^{405}$ When inhibition of factor Xa activity was compared after once, twice, or three times daily administration of the 5-mg dose, there was no detectable difference between the maximum effect on the first and the last day of administration. The plasma concentrations of rivaroxaban were also dose dependent, with maximum concentrations at 3 to $4 \mathrm{~h}$ for all doses and regimens and a half-life of approximately 4 to $6 \mathrm{~h}$ on the first day and of approximately 6 to $9 \mathrm{~h}$ on the last day of treatment. The correlation between plasma concentrations of rivaroxaban and inhibition of factor Xa activity or PT prolongation was linear, with an $r$ of 0.950 and 0.958 , respectively. ${ }^{405}$

Data from this study were later used for population modeling that would predict the behavior of the drug in patients. ${ }^{406}$ Results of this model showed that pharmacokinetics of rivaroxaban were linear and dose proportional up to the $30-\mathrm{mg}$ dose and confirmed a strong linear correlation between plasma concentration and pharmacodynamic parameters.

The pharmacokinetics and pharmacodynamics of rivaroxaban were also measured in a population of healthy elderly subjects and in patients with an extremely low or extremely high body weight (Table 4).407,408 In the first study, healthy subjects aged $\geq 60$ years received daily doses ranging between $30 \mathrm{mg}$ and $50 \mathrm{mg}$ after a standard breakfast. ${ }^{407}$ It was intended to assess even higher doses of up to $80 \mathrm{mg}$, but dose escalation was stopped prematurely because of an apparent ceiling effect in pharmacokinetic and pharmacodynamic variables. Maximum inhibition of factor Xa activity ranged from $68.4 \%$ after the $30-\mathrm{mg}$ dose to $75.3 \%$ and $74.5 \%$ after the doses of $40 \mathrm{mg}$ and $50 \mathrm{mg}$, respectively, and occurred 2 to $4 \mathrm{~h}$ after administration. No gender differences were observed. PT prolongation was also greater with the 40-mg dose than with the $30-\mathrm{mg}$ dose, and was not different between the two highest doses, with a maximum effect 2 to $3 \mathrm{~h}$ after drug administration. Similar profiles were documented for the aPTT and the HEPTEST. Maximum concentration of rivaroxaban was reached after $4 \mathrm{~h}$ in all dose groups, and the half-life

Table 4-[Section 3.2] Pharmacokinetic Characteristics of Rivaroxaban ${ }^{402,404,405}$

\begin{tabular}{|c|c|c|c|c|}
\hline Population and Dosage & Tmax, h, median & Cmax, $\mu \mathrm{g} / \mathrm{L}$ & $\mathrm{AUC}$ or $\mathrm{AUC} \tau, \mu \mathrm{g} \cdot \mathrm{h} / \mathrm{L}$ & $t_{1 / 2}, h$ \\
\hline \multicolumn{5}{|l|}{ Healthy adults ${ }^{\mathrm{a}}$} \\
\hline $5 \mathrm{mg}$ od & 3.00 & $76.4(18.3)$ & $505.5(19.7)$ & $8.4(32.6)$ \\
\hline $5 \mathrm{mg}$ bid & 3.00 & $85.3(17.7)$ & $458.5(13.1)$ & $7.0(27.8)$ \\
\hline $5 \mathrm{mg}$ tid & 2.00 & $123.8(19.7)$ & $557.3(20.4)$ & $5.8(35.5)$ \\
\hline $10 \mathrm{mg}$ bid & 2.98 & $158.0(18.8)$ & $863.8(18.6)$ & $7.6(26.7)$ \\
\hline $20 \mathrm{mg}$ bid & 2.50 & $318.1(18.7)$ & $1,903.0(24.5)$ & $8.0(40.7)$ \\
\hline $30 \mathrm{mg}$ bid & 3.02 & $451.9(10.5)$ & $2,728.0(14.6)$ & $9.2(64.1)$ \\
\hline \multicolumn{5}{|l|}{ Healthy elderly } \\
\hline $30 \mathrm{mg}$ single dose & 4.0 & $392.0(23.0)$ & $3,531.0(20.0)$ & $11.7(63.7)$ \\
\hline $40 \mathrm{mg}$ single dose & 4.0 & $461.0(16.8)$ & $4,385.0(24.1)$ & $13.3(31.9)$ \\
\hline $50 \mathrm{mg}$ single dose & 4.0 & $437.0(32.0)$ & $4,496.0(33.9)$ & $11.9(47.8)$ \\
\hline \multicolumn{5}{|l|}{ Body weight ${ }^{b}$} \\
\hline$\leq 50 \mathrm{~kg}$ women only & 4.00 & $178.1(16.6)$ & $1,172.0(22.0)$ & $9.6(36.7)$ \\
\hline $70-80 \mathrm{~kg}$ & 3.50 & $143.4(26.5)$ & $1,029(20.1)$ & $7.2(42.1)$ \\
\hline$>120 \mathrm{~kg}$ & 4.00 & $149.0(20.4)$ & $1,155(15.6)$ & $7.3(25.4)$ \\
\hline
\end{tabular}

Data are presented as geometric mean (geometric coefficient of variation). AUC $\tau=$ AUC during a dosage interval at steady state. See Table 3 for expansion of other abbreviations.

aMultiple doses.

b10 mg single dose. 
ranged between 12 and $13 \mathrm{~h}$ (Table 4). Excretion via the kidneys was similar among the dosing groups: although women had higher drug concentrations than men after the 30-mg and 40-mg doses, the authors considered that none of the pharmacokinetic parameters showed clinically significant differences between females and males. Finally, the slopes of correlations between plasma concentrations of rivaroxaban and clotting tests were similar in this population of healthy elderly subjects and the population of healthy young men enrolled in a previous study. ${ }^{407}$ Other studies have subsequently confirmed the absence of genderbased differences in the pharmacokinetics of rivaroxaban. ${ }^{409}$ Pharmacokinetics and pharmacodynamics of rivaroxaban in young and elderly Chinese men and women are comparable to those previously reported in studies of healthy whites. ${ }^{410,411}$

The effects of extreme body weight on a single 10 -mg daily dose of rivaroxaban were assessed in a study carried out in men and women aged 18 to 55 years weighing $\leq 50 \mathrm{~kg}$, between 70 and $80 \mathrm{~kg}$, or $>120 \mathrm{~kg}$ (Table 4). ${ }^{408}$ Peak plasma concentrations were higher in the low body weight group than in the other two groups, with a Cmax value that was significantly increased by $24 \%$ as compared with the normal body weight group. On the other hand, there was no difference in plasma concentrations between subjects with a normal or high body weight, and the AUC was similar among all three groups. The half-life of rivaroxaban was increased by $2 \mathrm{~h}$ in the low body weight group. Inhibition of factor Xa activity was similar in the three groups, with a maximum effect that occurred after 3 to $4 \mathrm{~h}$ and was slightly lower in the group of patients weighing $>120 \mathrm{~kg}$ than in the other two groups. The maximum prolongation of PT, aPTT, and the HEPTEST decreased significantly with increasing body weight, although overall all these changes were small. ${ }^{408}$

Finally, to define the pharmacokinetics and pharmacodynamics of rivaroxaban in a population of patients undergoing major orthopedic surgery, blood samples were obtained from patients enrolled in phase II randomized trials. ${ }^{412,413}$ The first study analyzed data from two trials that assessed bid doses of rivaroxaban ranging from $2.5 \mathrm{mg}$ to $30 \mathrm{mg}$ administered at mealtimes. ${ }^{412}$ Exclusion criteria from these trials were body weight $<45 \mathrm{~kg}$, creatinine clearance $<30 \mathrm{~mL} / \mathrm{min}$, and severely impaired hepatic function. The authors found substantial variability in the pharmacokinetics of rivaroxaban on the first postoperative day for all doses, possibly attributed to the presence of "slow" and "fast" absorbers. Overall, absorption was fast, with a Cmax within 1 to $2 \mathrm{~h}$. Absorption status was unrelated to gender, food, anesthesia, or the use of comedications. The variability at steady state was only moderate, and the increase of exposure was dose dependent. Between $14 \%$ and $31 \%$ of rivaroxaban was excreted unchanged via the kidneys. The clearance of the drug was consistently affected only by renal function, with a creatinine clearance of $30 \mathrm{~mL} / \mathrm{min}$ resulting in a $15 \%$ to $35 \%$ higher drug exposure in these two trials. ${ }^{412}$ Based on the results of subsequent simulations of various scenarios for patients with different extremes of covariates, it was estimated that plasma concentrations of rivaroxaban would have exceeded the $90 \%$ CI of the average patient only in a 90-year-old patient weighing $30 \mathrm{~kg}$, suggesting that one dose of the drug could be administered to all patients regardless of their age, gender, and body weight. The second study compared data from two phase II clinical trials, both carried out in patients undergoing total hip replacement surgery, one assessing bid doses and the other assessing single daily doses. ${ }^{413}$ Overall, the pharmacokinetics were similar after once-daily or bid dosing, since only the steadystate area under the plasma concentration-time curve over $24 \mathrm{~h}$ was higher with once-daily doses than with bid doses ${ }^{413}$ (Table S4). PT prolongation strongly correlated with plasma concentrations when rivaroxaban was given once daily or bid. ${ }^{413}$ Simulations of plasma concentration expected after a 10-mg once-daily dose in model subjects with extreme demographic characteristics showed that plasma concentrationtime profiles would fall within the predicted $90 \%$ CI of the average patient.

\subsection{Interactions}

3.3.1 Drugs: In humans, CYP3A4 plays a pivotal role in the oxidative metabolism of rivaroxaban. ${ }^{401}$ Drugs that inhibit or induce CYP3A4 have the potential to interact with rivaroxaban. However, only drugs that act as strong inhibitors of both CYP3A4 and of P-glycoprotein, a transporter protein of which rivaroxaban is a substrate, have been shown to cause important reduction of the clearance of the drug, thus provoking a significant increase in plasma concentrations. These drugs include azole antimycotics and HIV protease inhibitors. The concomitant administration of rivaroxaban with ketoconazole $400 \mathrm{mg}$ once daily or with ritonavir $600 \mathrm{mg}$ bid resulted in an approximately 2.5 -fold increase in the mean AUC and 1.7-fold increase in the mean Cmax of rivaroxaban, together with significantly increased effects on clotting tests. ${ }^{414}$ Thus, the use of rivaroxaban in patients receiving ketoconazole, itraconazole, voriconazole, and posaconazole or any HIV protease inhibitor is currently not recommended. ${ }^{414}$ Rivaroxaban should be used with caution when given together with other drugs that strongly inhibit only CYP3A4 or only P-glycoprotein. These drugs include clarithromycin, which at the dose of $500 \mathrm{mg}$ bid leads to a 
1.5-fold increase in the mean AUC and to a 1.4-fold increase in the Cmax of the drug, and erythromycin, which at the dose of $500 \mathrm{mg}$ three times daily causes a 1.3-fold increase in both the AUC and the Cmax. ${ }^{414}$ On the other hand, reduced plasma concentrations of rivaroxaban can occur when strong CYP3A4 inducers are coadministered. These include rifampicin (which causes a $50 \%$ decrease in the AUC), phenytoin, carbamazepine, phenobarbital, or St. John's wort. ${ }^{414}$ The concomitant administration of rivaroxaban with substrates of either CYP3A4 or P-glycoprotein, such as atorvastatin, digoxin, or midazolam, did not result in clinically relevant interactions. ${ }^{415,416}$

The concomitant administration of rivaroxaban and aspirin was tested in a randomized, two-way crossover study. ${ }^{403}$ Healthy men aged between 18 and 55 years were randomized to receive aspirin alone at the dose of $500 \mathrm{mg}$ on the first day and $100 \mathrm{mg}$ on the second day, rivaroxaban alone at the dose of $15 \mathrm{mg}$, or aspirin and rivaroxaban at the same dosages. Patients receiving rivaroxaban alone were subsequently treated with rivaroxaban and aspirin and vice versa. Maximum levels of inhibition of factor Xa activity and maximum prolongation of the PT, aPTT, and the HEPTEST were similar in patients treated with rivaroxaban alone and in patients treated with rivaroxaban plus aspirin. Collagen-stimulated platelet aggregation was inhibited in the aspirin-alone group and in the aspirin plus rivaroxaban group but not in the rivaroxaban-alone group. Inhibition of platelet aggregation with aspirin was $89.3 \%$ greater than with rivaroxaban alone, and with aspirin plus rivaroxaban it was $97.4 \%$ greater than with rivaroxaban alone. Bleeding time was not affected by rivaroxaban alone, but was prolonged to 1.46 times the baseline by aspirin alone and to 1.96 times the baseline by adding aspirin to rivaroxaban. The combination of aspirin with rivaroxaban prolonged bleeding time more than aspirin alone. Pharmacokinetic parameters of rivaroxaban were not altered by the coadministration of aspirin.

The concomitant administration of rivaroxaban and a nonsteroidal antiinflammatory drug was also tested in a randomized two-way crossover study. ${ }^{417}$ Healthy men aged between 18 and 45 years were randomized to receive $15 \mathrm{mg}$ of rivaroxaban alone or $15 \mathrm{mg}$ rivaroxaban plus naproxen $500 \mathrm{mg}$. After 14 days all subjects crossed over. Maximum inhibition of factor Xa activity and maximum prolongations of the PT, aPTT, and HEPTEST were similar between the two groups. Bleeding time was significantly increased by the combined use of rivaroxaban and naproxen as compared with rivaroxaban alone. Plasma concentrations of rivaroxaban were slightly increased, by $10 \%$ for both the AUC and the Cmax after the coadministration of naproxen. Finally, the concomitant administration of drugs that alter the gastric $\mathrm{pH}$, such as the histamine $\mathrm{H} 2$ antagonist ranitidine or the antacid aluminummagnesium hydroxide, had no effect on the plasma concentrations or pharmacodynamics of rivaroxaban. ${ }^{418}$

3.3.3 Environmental Factors: The effect of food on the absorption of rivaroxaban was tested in healthy male subjects aged 18 to 45 years. ${ }^{418}$ After a single dose of $5 \mathrm{mg}$, the absorption of rivaroxaban was found to be slower in the fed state than the fasting state, with a delay in the median time to reach the peak plasma concentration from $2.75 \mathrm{~h}$ to $4.0 \mathrm{~h}$. The AUC and the Cmax both increased with the concomitant administration of food, by $28 \%$ and $41 \%$, whereas the terminal half-life remained unchanged. The fed state increased the time to maximum prolongation of the PT and maximum inhibition of factor Xa activity and also the maximum effect on these clotting tests. Thus, absorption of rivaroxaban was moderately increased after the administration of food, with a resulting increase in pharmacokinetic and pharmacodynamic parameters. In addition, concomitant food intake reduced interindividual variability, whereas elimination remained unchanged. No pharmacokinetic differences were documented when high-fat, highcalorie meals were compared with high-carbohydrate meals. These food effects have been attributed to a prolonged length of stay in the stomach that is possibly related to the lipophilicity and limited aqueous solubility of the drug.418

\subsection{Antithrombotic Effect}

Rivaroxaban acts as a competitive inhibitor of the amidolytic activity of factor Xa. In vitro, the inhibitory effect is concentration dependent, with an inhibitory constant against factor Xa of $0.4 \pm 0.02 \mathrm{nM} .{ }^{399}$ The selectivity of rivaroxaban for factor $\mathrm{Xa}$ is 10,000 -fold greater than for other serine proteases, including factor Va, factor IXa, factor XIa, thrombin, and activated protein $\mathrm{C}$. The onset of action is rapid and reversible, as shown by the kinetic association rate constant of $1.7 \times 10^{7} \mathrm{~mol} / \mathrm{L}^{-1} / \mathrm{s}^{-1}$ and kinetic dissociation rate constant of $5 \times 10^{-3} / \mathrm{s}^{-1}$. 419 The inhibitory effect is maintained when factor $\mathrm{Xa}$ is complexed with factor $\mathrm{Va}$ and $\mathrm{Ca}^{2+}$ on a phospholipid membrane (prothrombinase bound) as shown by a concentration-dependent inhibition of thrombin generation ${ }^{399}$ and also when factor $\mathrm{Xa}$ is bound to the clot. ${ }^{420}$

In plasma, endogenous human factor $\mathrm{Xa}$ is inhibited by rivaroxaban to cause dose-dependent prolongation of both the PT and aPTT, with the PT being more sensitive than the aPTT. ${ }^{399}$ In whole blood and in platelet-rich plasma, rivaroxaban has a dosedependent effect in prolonging the initiation phase of thrombin generation after activation of the tissue 
factor pathway, reduces the maximum concentration of generated thrombin, and decreases the endogenous thrombin potential. ${ }^{400}$ The effect on the initiation and propagation phases of thrombin generation is greater than the effect on the decay phase, expressed by the endogenous thrombin potential. High concentrations of rivaroxaban are able to prevent thrombin generation almost completely, due to inhibition of factor Xa bound to the prothrombinase complex. ${ }^{400}$ The effect of rivaroxaban on platelet-induced thrombin generation in vivo was tested in a randomized, placebo-controlled, crossover study of 12 healthy male subjects aged 27 to 37 years. ${ }^{397}$ The prothrombinaseinduced clotting time, a plasma clotting assay based on the activation of coagulation using factor $\mathrm{Xa}$, phospholipids, and an enzyme that activates factor $\mathrm{V}$, was prolonged dose dependently by rivaroxaban, with a maximum effect after $2 \mathrm{~h}$. Thrombin generation in platelet-rich plasma was markedly reduced by rivaroxaban, with an $80 \%$ to $90 \%$ decrease (by $5-\mathrm{mg}$ and 30-mg doses, respectively) of the peak collageninduced endogenous thrombin potential at $2 \mathrm{~h}$. There was a close correlation between plasma concentration of rivaroxaban and its effects on factor Xa activity inhibition, prothrombinase-induced clotting time prolongation, and endogenous thrombin potential reduction. ${ }^{397}$

Rivaroxaban has no effects on platelet aggregation induced by collagen, adenosine diphosphate, or thrombin. ${ }^{403,421,422}$ An indirect effect on platelet aggregation induced by tissue factor, determined by the inhibition of thrombin generation, has been reported in defibrinated plasma. ${ }^{423}$

In venous thrombosis models and in arteriovenous shunt models, rivaroxaban was shown to cause dosedependent reduction of thrombus formation, inhibition of factor Xa activity, and prolongation of the PT. ${ }^{399}$ The antithrombotic effective doses of rivaroxaban did not prolong bleeding time in animal bleeding models. ${ }^{399}$

\subsection{Monitoring Anticoagulant Intensity}

The predictable pharmacologic profile of rivaroxaban allows the administration of the drug at fixed doses without the need for routine laboratory monitoring or dose adjustments. However, there may be rare situations, as in the case of overdose or unexpected bleeding, assessment of compliance, evaluation of drug interactions, or assessment of drug accumulation in renal or hepatic impairment, when the availability of a quantitative clotting assay might be valuable. Despite the predictable, dose-dependent effects of rivaroxaban on the PT and (to a lesser extent) the aPTT, and on tests measuring thrombin generation, there are currently no validated labora- tory assays that can be recommended to monitor rivaroxaban or any recommendations for dose adjustments based on observed test results. For instance, the thromboplastins used for PT clotting assays have differing sensitivities to factor Xa inhibitors, and the INR introduced to correct for differences in PT sensitivity when monitoring the VKAs does not adequately correct for differences in assay sensitivity to direct factor Xa inhibitors. Smith and Morissey ${ }^{424}$ evaluated the effects of five commercial thromboplastin reagents on sensitivity of the PT to rivaroxaban and its correlation with the INR. PT ratios (ie, PT with drug/PT without drug) were measured using normal human plasma to which rivaroxaban $1 \mu \mathrm{g} / \mathrm{mL}$ was added in vitro. PT ratios varied from 2.25 to 7.32 with the different thromboplastins; subsequent conversion to an INR further exacerbated the observed differences in sensitivities to rivaroxaban between the various PT assays.

Recently, Samama et $\mathrm{al}^{402}$ carried out a study that aimed to identify a clotting test suitable for monitoring rivaroxaban activity by evaluating the effects on a number of different assays of increasing the drug concentration in pooled citrated normal human platelet-poor plasma. There was a concentrationdependent prolongation of the PT and aPTT, but the increases in clotting times varied depending on the thromboplastin reagent used. ${ }^{402}$ The effect of rivaroxaban on the aPTT was weaker than that on the PT. Rivaroxaban also prolonged the thromboelastograph parameters. Standard methods for the HEPTEST and the prothrombinase-induced clotting time resulted in paradoxical responses. Tests used to measure antifactor Xa activity of the low-molecular-weight heparins showed dose-dependent effects but were associated with some degrees of variation. Finally, rivaroxaban also caused a dose-dependent increase of the diluted Russell viper venom ratio. Specific calibration of some of these tests may lead to the availability of an appropriate assay to monitor the pharmacodynamic effects of the drug. Neither the PT (expressed either in seconds or as a ratio) nor the aPTT should be used to monitor the anticoagulant effect of rivaroxaban.

\subsection{Practical Issues Related to Initiation and Maintenance}

Rivaroxaban is currently approved in many countries for the prevention of VTE in patients undergoing total hip replacement surgery and total knee replacement surgery based on the results of four phase 3 clinical trials. ${ }^{393-396}$ For this indication, the approved dose is $10 \mathrm{mg}$ once daily. Treatment should be started between 6 and $10 \mathrm{~h}$ after surgery, and the duration of treatment should vary from 2 weeks in patients undergoing total knee replacement surgery 
to 5 weeks in patients undergoing total hip replacement surgery, although approved durations may vary among countries. Rivaroxaban is currently not approved for use in patients with severe renal failure and a creatinine clearance of $<15 \mathrm{~mL} / \mathrm{min}$, in patients with hepatic disease associated with coagulopathy, and in patients receiving concomitant systemic treatment with azole-antimycotics or HIV protease inhibitors. ${ }^{414}$ Rivaroxaban is also not approved for use in children or adolescents $<18$ years of age because of the absence of clinical data, in pregnant women because of the potential for reproductive toxicity (observed in animals) and the evidence that the drug passes the placenta, and during breast-feeding because the drug is secreted into milk. ${ }^{414}$

Future additional indications are expected following the results of recently published clinical trials. In particular, more information on the long-term administration of rivaroxaban now originates from studies carried out in patients with VTE or AF in whom the drug was administered for a minimum of 6 months and up to a maximum of 40 months. ${ }^{425,426}$

To date, the results of a phase III clinical trials assessing the use of rivaroxaban in the treatment of DVT have been published. In the EINSTEIN study, $>3,400$ patients with acute symptomatic proximal DVT were allocated to rivaroxaban $15 \mathrm{mg}$ bid for 3 weeks followed by $20 \mathrm{mg}$ once daily or initial therapy with enoxaparin $(1 \mathrm{mg} / \mathrm{kg}$ bid $)$ and simultaneous warfarin administered to a target INR of 2.0 to 3.0. ${ }^{425}$ The primary outcome measure was symptomatic recurrent VTE, which occurred in 36 rivaroxaban-treated patients and 51 low-molecularweight heparin/warfarin-treated patients (HR, 0.68; 95\% CI, 0.44-1.04; $P<.0001$ for noninferiority). Patients who had completed 6 to 12 months of anticoagulant treatment with either a VKA or rivaroxaban (if also enrolled in the acute-phase rivaroxaban treatment study) after an acute episode of VTE were randomized to receive rivaroxaban $20 \mathrm{mg}$ once daily or matching placebo for additional 6 to 12 months. The study showed superior efficacy of rivaroxaban over placebo in the prevention of recurrent venous thromboembolic events, a nonsignificant increase in major bleeding, and an increase in clinically relevant, nonmajor bleeding events. ${ }^{425}$

More recently, the results of the Rivaroxaban Once Daily Oral Direct Factor Xa inhibition Compared with Vitamin K Antagonist for the Prevention of Stroke and Embolism Trial in Atrial Fibrillation (ROCKET) study comparing rivaroxaban $20 \mathrm{mg}$ once daily (15 mg once daily if patients had a creatinine clearance between 30 and $49 \mathrm{~mL} / \mathrm{min}$ ) with warfarin in patients with $\mathrm{AF}$ and at least two additional risk factors for embolic events were published. In this randomized, double-blind, controlled trial, rivaroxa- ban was noninferior to warfarin in the prevention of ischemic stroke or systemic embolism, with a similar rate of major bleeding events but with fewer intracranial and fatal bleeding events. ${ }^{426}$

Other recently completed phase III trials include a study on the prevention of VTE in medical patients (Multicenter, Randomized, Parallel Group Efficacy and Safety Study for the Prevention of VTE in Hospitalized Medically Ill Patients Comparing Rivaroxaban with Enoxaparin [MAGELLAN] study), in which rivaroxaban is administered at the same $10 \mathrm{mg}$ oncedaily dose used in the Regulation of Coagulation in Major Orthopedic Surgery Reducing the Risk of DVT and PE (RECORD) program; and the EINSTEIN PE study, in which patients with hemodynamically stable $\mathrm{PE}$ are treated for the first 3 weeks with a $15 \mathrm{mg}$ bid dose of rivaroxaban followed by a $20 \mathrm{mg}$ once-daily dose. Finally, a phase III study in patients with acute coronary syndromes is assessing rivaroxaban doses of $2.5 \mathrm{mg}$ and $5.0 \mathrm{mg}$ bid. ${ }^{427}$

\subsection{Adverse Events}

3.7.1 Bleeding Events: Data on the incidence of bleeding complications with the use of rivaroxaban are currently available from the results of phase II and phase III clinical trials only. As of yet, no published phase IV studies or reports from clinical practice have been published. Reported rates must take into account the definition of bleeding used in each study, given the variability of definitions used among clinical trials assessing different compounds.

The reported rates of any bleeding with rivaroxaban in the four phase III clinical trials carried out in patients undergoing total hip and total knee replacement surgery range from $4.9 \%$ to $10.5 \%^{393-396}$ and are comparable to the rates observed in patients treated with enoxaparin. Rates of major bleeding events vary from $<0.1 \%$ to $0.7 \%$, and the rates of clinically relevant nonmajor bleeding events vary from $2.6 \%$ to $3.3 \%$, again with no statistically significant differences between the rates observed in the rivaroxaban groups and the rates observed in the comparator groups (ie, the low-molecular-weight heparin enoxaparin in all studies). ${ }^{393-396}$ A pooled analysis of the four RECORD studies reported that the concomitant use of nonsteroidal antiinflammatory drugs, aspirin, or other antiplatelet agents was not associated with an increased rate of bleeding events. ${ }^{428}$

The rate of any bleeding event in patients with a previous episode of VTE treated with rivaroxaban $20 \mathrm{mg}$ once daily for 6 to 12 months after the initial standard course of anticoagulant therapy was $23 \%$; major bleeding and clinically relevant nonmajor bleeding occurred in $0.7 \%$ and $5.4 \%$ of patients, respectively. ${ }^{425}$ 
3.7.2 Nonhemorrhagic Adverse Events: None of the four phase III clinical trials found evidence of drug-associated liver toxicity in patients treated with rivaroxaban for up to 5 weeks. ${ }^{393-396}$ Overall, the proportion of patients with elevated liver enzymes was low in all studies and was similar between rivaroxaban and enoxaparin. The incidence of cardiovascular events was low while on treatment with rivaroxaban, ranging between $0.1 \%$ and $0.7 \%$, and was similar to that seen with enoxaparin. Drug-related adverse events occurred in $12 \%$ to $20 \%$ of patients in the four RECORD trials; these rates were similar to those observed with enoxaparin. The most frequent adverse events were nausea, vomiting, and constipation.

\subsection{Reversal}

There is currently no specific antidote available to antagonize the effects of rivaroxaban. In case of overdose, the use of activated charcoal to reduce absorption is suggested. ${ }^{414}$ Because of the high plasma protein binding, rivaroxaban is unlikely to be dialyzable. In case of active bleeding, possible strategies currently include discontinuation of treatment and administration of blood products or component transfusion if required to treat an identified deficiency. ${ }^{414}$ However, there is currently no direct evidence in humans to support the efficacy of blood product transfusion or other interventions in improving hemostasis when patients have received rivaroxaban. Recently, Perzborn et al ${ }^{429}$ reported the results of a study carried out in rats treated with high-dose rivaroxaban, which aimed to assess the efficacy of prothrombin complex concentrate. After determination of baseline mesenteric bleeding time, rats were initially treated with IV rivaroxaban and subsequently received fourfactor prothrombin complex concentrates at $25 \mathrm{U} / \mathrm{kg}$ or $50 \mathrm{U} / \mathrm{kg}$. Prolongation of the bleeding time was almost completely abrogated by higher dose of prothrombin complex concentrates, whereas the lower dose was ineffective. The use of recombinant factor VIIa is also suggested in the presence of life-threatening bleeding based on some preclinical data. ${ }^{414} \mathrm{~A}$ reconstructed recombinant factor $\mathrm{Xa}$ has been recently proposed as a potential antidote for factor Xa inhibitors. ${ }^{430}$ This is a catalytically inactive factor Xa that has no procoagulant or anticoagulant activity and does not interfere with the prothrombinase complex but maintains high affinity for factor Xa inhibitors. In plasma, the addition of the antidote dose-dependently reversed factor Xa inhibition as measured by anti-factor Xa units, tissue factor-initiated thrombin generation, and clotting assays. In vivo, the antidote completely reversed PT prolongation induced by intravenous infusion of rivaroxaban in rats.

\section{CONCLUSION}

Over the last decades, a large amount of research has been addressed to improve the understanding of the mechanisms of warfarin, acenocoumarol, and phenprocoumon and to improve the management of patients treated with these VKAs. Several studies have in particular identified some genetic factors associated with the individual responses to VKAs and several drugs, foods, and environmental factors that can interact with these compounds. Several induction and maintenance strategies have been compared, and management studies have evaluated different approaches for the monitoring of patients on VKAs, including AMS and AC, computer programs, and POC for INR testing. The results of all such studies have greatly contributed to improve the efficacy and safety of oral anticoagulant therapy and to increase the number of patients who can be deemed eligible for such treatment. Finally, a number of trials have also addressed the management of patients on VKA treatment who are at increased risk of bleeding or are actively bleeding, and a number of therapeutic strategies have been proposed, although additional research may be warranted in particular to further improve the management of the bleeding patient.

The new oral anticoagulant drugs have the potential to overcome several drawbacks of the VKAs. These drugs can be administered at fixed doses and do not require laboratory monitoring, thus offering a clear advantage over the VKAs. It is hoped that new studies will provide us with further information on the role of specific laboratory tests for the monitoring of the activity of these new classes of drugs, when requested, and on the optimal management of drugrelated adverse events.

\section{ACKNOWLEDGMENTS}

Author contributions: As Topic Editor, Dr Ageno oversaw the development of this article, including any analysis and subsequent development of the information contained herein.

Dr Ageno: contributed as Topic Editor.

Dr Gallus: contributed as a panelist.

Dr Wittkowsky: contributed as a panelist.

Dr Crowther: contributed as a panelist.

Dr Hylek: contributed as a panelist.

Dr Palareti: contributed as a panelist.

Financial/nonfinancial disclosures: In summary, the authors have reported to CHEST the following conflicts of interest: Dr Ageno has received fees for steering committee or safety advisory boards membership from Bayer Health Care, sanofi-aventis, and Thrombogenics; research grants from GlaxoSmithKline, Alexion Pharmaceutical, Pfizer, and Bayer Health Care; honoraria for speaking activities at national or international meetings and for advisory boards from Bayer Health Care, GlaxoSmithKline, sanofi-aventis, Pfizer, BMS, and Boehringer Ingelheim. Dr Gallus has received fees for steering committee membership from BMS, Pfizer, sanofiaventis, Bayer, ASTELLAS, Daiichi Sankyo. Dr Crowther has served on various advisory boards, has assisted in the preparation of educational materials, has sat on data and safety monitoring boards, and his institution has received research funds from the following companies: Leo Pharma, Pfizer, Boehringer Ingelheim, 
Bayer, Octapharm, CSL Behring, and Artisan. His personal total compensation for these activities over the last 3 years totals less than $\$ 10,000$. Dr Hylek has served on advisory boards (Bayer, Boehringer Ingelheim, Bristol-Myers Squibb, Daiichi Sankyo, Johnson \& Johnson, Merck, Ortho-McNeil, and Pfizer; total dollar amount less than $\$ 10,000)$. She has served on the Executive Steering Committee-ARISTOTLE trial sponsored by Bristol-Myers Squibb and Pfizer (dollar amount less than $\$ 10,000$ ). She has also served on the Executive Steering Committee-ORBIT AF Registry sponsored by Ortho-McNeil (dollar amount less than $\$ 10,000$ ). She has participated in a symposium sponsored by Boehringer Ingelheim (dollar amount less than $\$ 10,000)$. Drs Wittkowsky and Palareti have reported to CHEST that no potential conflicts of interest exist with any companies/organizations whose products or services may be discussed in this article.

Role of sponsors: The sponsors played no role in the development of these guidelines. Sponsoring organizations cannot recommend panelists or topics, nor are they allowed prepublication access to the manuscripts and recommendations. Guideline panel members, including the chair, and members of the Health \& Science Policy Committee are blinded to the funding sources. Further details on the Conflict of Interest Policy are available online at http://chestnet.org

Endorsements: This guideline is endorsed by the American Association for Clinical Chemistry, the American College of Clinical Pharmacy, the American Society of Health-System Pharmacists, the American Society of Hematology, and the International Society of Thrombosis and Hematosis.

Additional information: The supplement Tables can be found in the Online Data Supplement at http://chestjournal.chestpubs. org/content/141/2_suppl/e44S/suppl/DC1.

\section{REFERENCES}

1. Whitlon DS, Sadowski JA, Suttie JW. Mechanism of coumarin action: significance of vitamin $\mathrm{K}$ epoxide reductase inhibition. Biochemistry. 1978;17(8):1371-1377.

2. Fasco MJ, Hildebrandt EF, Suttie JW. Evidence that warfarin anticoagulant action involves two distinct reductase activities. J Biol Chem. 1982;257(19):11210-11212.

3. Choonara IA, Malia RG, Haynes BP, et al. The relationship between inhibition of vitamin K1 2,3-epoxide reductase and reduction of clotting factor activity with warfarin. $\mathrm{Br} \mathrm{J} \mathrm{Clin}$ Pharmacol. 1988;25(1):1-7.

4. Trivedi LS, Rhee M, Galivan JH, Fasco MJ. Normal and warfarin-resistant rat hepatocyte metabolism of vitamin $\mathrm{K}$ 2,3-epoxide: evidence for multiple pathways of hydroxyvitamin K formation. Arch Biochem Biophys. 1988;264(1):67-73.

5. Stenflo J, Fernlund P, Egan W, Roepstorff P. Vitamin K dependent modifications of glutamic acid residues in prothrombin. Proc Natl Acad Sci U S A. 1974;71(7):2730-2733.

6. Nelsestuen GL, Zytkovicz TH, Howard JB. The mode of action of vitamin $\mathrm{K}$. Identification of gamma-carboxyglutamic acid as a component of prothrombin. J Biol Chem. 1974; 249(19):6347-6350

7. Stafford DW. The vitamin K cycle. J Thromb Haemost. 2005;3(8):1873-1878

8. Ansell J, Hirsh J, Hylek E, Jacobson A, Crowther M, Palareti G; American College of Chest Physicians. Pharmacology and management of the vitamin $\mathrm{K}$ antagonists: American College of Chest Physicians Evidence-Based Clinical Practice Guidelines (8th Edition). Chest. 2008; 133(suppl 6):160S-198S.

9. Friedman PA, Rosenberg RD, Hauschka PV, Fitz-James A. A spectrum of partially carboxylated prothrombins in the plasmas of coumarin-treated patients. Biochim Biophys Acta. 1977;494(1):271-276.

10. Malhotra OP, Nesheim ME, Mann KG. The kinetics of activation of normal and gamma-carboxyglutamic acid-deficient prothrombins. J Biol Chem. 1985;260(1):279-287.
11. Nelsestuen GL, Zytkovicz TH, Howard JB. Role of gammacarboxyglutamic acid. An unusual protein transition required for the calcium-dependent binding of prothrombin to phospholipid. J Biol Chem. 1976;251(18):5648-5656.

12. Prendergast FG, Mann KG. Differentiation of metal ioninduced transitions of prothrombin fragment 1. J Biol Chem. 1977;252(3):840-850.

13. Borowski M, Furie BC, Bauminger S, Furie B. Prothrombin requires two sequential metal-dependent conformational transitions to bind phospholipid. Conformation-specific antibodies directed against the phospholipid-binding site on prothrombin. J Biol Chem. 1986;261(32):14969-14975.

14. Becker R. The importance of factor Xa regulatory pathways in vascular thromboresistance: focus on protein $\mathrm{Z}$. J Thromb Thrombolysis. 2005;19(2):135-137.

15. Breckenridge A. Oral anticoagulant drugs: pharmacokinetic aspects. Semin Hematol. 1978;15(1):19-26.

16. O'Reilly RA. Vitamin K and the oral anticoagulant drugs. Annu Rev Med. 1976;27:245-261.

17. Kelly JG, O'Malley K. Clinical pharmacokinetics of oral anticoagulants. Clin Pharmacokinet. 1979;4(1):1-15.

18. O'Reilly R, ed. Warfarin Metabolism and Drug-Drug Interactions. New York, NY: Plenum; 1986.

19. Miners JO, Birkett DJ. Cytochrome P4502C9: an enzyme of major importance in human drug metabolism. Br J Clin Pharmacol. 1998;45(6):525-538.

20. Godbillon J, Richard J, Gerardin A, Meinertz T, Kasper W, Jähnchen E. Pharmacokinetics of the enantiomers of acenocoumarol in man. Br J Clin Pharmacol. 1981;12(5):621-629.

21. Haustein KO. Pharmacokinetic and pharmacodynamic properties of oral anticoagulants, especially phenprocoumon. Semin Thromb Hemost. 1999;25(1):5-11.

22. Mentré F, Pousset F, Comets E, et al. Population pharmacokinetic-pharmacodynamic analysis of fluindione in patients. Clin Pharmacol Ther. 1998;63(1):64-78.

23. Home page of the Human Cytochrome P450 (CYP) Allele Nomenclature Committee. Web site. http://www.CYPalleles. ki.se. Accessed August 31, 2010

24. Scordo MG, Pengo V, Spina E, Dahl ML, Gusella M, Padrini R. Influence of CYP2C9 and CYP2C19 genetic polymorphisms on warfarin maintenance dose and metabolic clearance. Clin Pharmacol Ther. 2002;72(6):702-710.

25. Wittkowsky AK. Pharmacology of warfarin and related anticoagulants. In: Ansell J, Oertel L, Wittkowsky A, eds. Managing Oral Anticoagulation Therapy: Clinical and Operational Guidelines. Vol. 1. St. Louis, MO: Facts and Comparisons; 2003;29(1)-29:15.

26. Loebstein R, Yonath H, Peleg D, et al. Individual variability in sensitivity to warfarin: nature or nurture. Clin Pharmacol Ther. 2001;70(2):159-164.

27. Lindh JD, Holm L, Andersson ML, Rane A. Influence of CYP2C9 genotype on warfarin dose requirements-a systematic review and meta-analysis. Eur J Clin Pharmacol. 2009;65(4):365-375

28. Aithal GP, Day CP, Kesteven PJ, Daly AK. Association of polymorphisms in the cytochrome P450 CYP2C9 with warfarin dose requirement and risk of bleeding complications. Lancet. 1999;353(9154):717-719.

29. Higashi M, Veenstra DL, Wittkowsky AK, et al. Influence of CYP2C9 genetic variants on the risk of over anticoagulation and of bleeding events during warfarin therapy. JAMA. 2002;287(13):1690-1698.

30. Veenstra DL, Blough DK, Higashi MK, et al. CYP2C9 haplotype structure in European American warfarin patients and association with clinical outcomes. Clin Pharmacol Ther. 2005;77(5):353-364 
31. Chern HD, Ueng TH, Fu YP, Cheng CW. CYP2C9 polymorphism and warfarin sensitivity in Taiwan Chinese. Clin Chim Acta. 2006;367(1-2):108-113.

32. Herman D, Peternel P, Stegnar M, Breskvar K, Dolzan V. A novel sequence variant in exon 7 of CYP2C9 gene (CYP2C9*24) in a patient on warfarin therapy. Thromb Haemost. 2006;95(1):192-194.

33. Visser LE, van Schaik RHN, van Vliet M, et al. The risk of bleeding complications in patients with cytochrome $\mathrm{P} 450$ CYP2C9*2 or CYP2C9*3 alleles on acenocoumarol or phenprocoumon. Thromb Haemost. 2004;92(1):61-66.

34. Takahashi H, Wilkinson GR, Padrini R, Echizen H. CYP2C9 and oral anticoagulation therapy with acenocoumarol and warfarin: similarities yet differences. Clin Pharmacol Ther. 2004; $75(5): 376-380$.

35. Beinema M, Brouwers JR, Schalekamp T, Wilffert B. Pharmacogenetic differences between warfarin, acenocoumarol and phenprocoumon. Thromb Haemost. 2008;100(6):1052-1057.

36. Zimmermann A, Matschiner JT. Biochemical basis of hereditary resistance to warfarin in the rat. Biochem Pharmacol. 1974;23(6):1033-1040.

37. Rost S, Fregin A, Ivaskevicius V, et al. Mutations in VKORC1 cause warfarin resistance and multiple coagulation factor deficiency type 2. Nature. 2004;427(6974):537-541.

38. Li T, Chang CY, Jin DY, Lin PJ, Khvorova A, Stafford DW. Identification of the gene for vitamin $\mathrm{K}$ epoxide reductase. Nature. 2004;427(6974):541-544.

39. D'Andrea G, D'Ambrosio RL, Di Perna P, et al. A polymorphism in the VKORC1 gene is associated with an interindividual variability in the dose-anticoagulant effect of warfarin. Blood. 2005;105(2):645-649.

40. Rieder MJ, Reiner AP, Gage BF, et al. Effect of VKORC1 haplotypes on transcriptional regulation and warfarin dose. $N$ Engl J Med. 2005;352(22):2285-2293.

41. Geisen C, Watzka M, Sittinger K, et al. VKORC1 haplotypes and their impact on the inter-individual and interethnical variability of oral anticoagulation. Thromb Haemost. 2005;94(4):773-779.

42. Sconce EA, Khan TI, Wynne HA, et al. The impact of CYP2C9 and VKORC1 genetic polymorphism and patient characteristics upon warfarin dose requirements: proposal for a new dosing regimen. Blood. 2005;106(7):23292333.

43. Harrington DJ, Underwood S, Morse C, Shearer MJ, Tuddenham EGD, Mumford AD. Pharmacodynamic resistance to warfarin associated with a Val66Met substitution in vitamin $\mathrm{K}$ epoxide reductase complex subunit 1 . Thromb Haemost. 2005;93(1):23-26.

44. Anthony M, Romero K, Malone DC, Hines LE, Higgins L, Woosley RL. Warfarin interactions with substances listed in drug information compendia and in the FDA-approved label for warfarin sodium. Clin Pharmacol Ther. 2009;86(4): 425-429.

45. Marsh S, King CR, Porche-Sorbet RM, Scott-Horton TJ, Eby CS. Population variation in VKORC1 haplotype structure. J Thromb Haemost. 2006;4(2):473-474.

46. Quteineh L, Verstuyft C, Descot C, et al. Vitamin K epoxide reductase (VKORC1) genetic polymorphism is associated to oral anticoagulant overdose. Thromb Haemost. 2005;94(3): 690-691.

47. Coumadin (Warfarin) [package insert]. Princeton NJ: Bristol Myers Squibb; 2007.

48. Holbrook AM, Pereira JA, Labiris R, et al. Systematic overview of warfarin and its drug and food interactions. Arch Intern Med. 2005;165(10):1095-1106.

49. Orme M, Breckenridge A. Enantiomers of warfarin and phenobarbital. N Engl J Med. 1976;295(26):1482-1483.
50. Breckenridge A, Orme M, Wesseling H, Lewis RJ, Gibbons R. Pharmacokinetics and pharmacodynamics of the enantiomers of warfarin in man. Clin Pharmacol Ther. 1974; 15(4):424-430.

51. O'Reilly RA. Studies on the optical enantiomorphs of warfarin in man. Clin Pharmacol Ther. 1974;16(2):348-354.

52. O'Reilly RA, Trager WF, Motley CH, Howald W. Stereoselective interaction of phenylbutazone with $\left[{ }^{12} \mathrm{C} /{ }^{13} \mathrm{C}\right]$ warfarin pseudoracemates in man. J Clin Invest. 1980;65(3): 746-753.

53. Toon S, Low LK, Gibaldi M, et al. The warfarinsulfinpyrazone interaction: stereochemical considerations. Clin Pharmacol Ther. 1986;39(1):15-24.

54. O'Reilly RA. The stereoselective interaction of warfarin and metronidazole in man. N Engl J Med. 1976;295(7):354-357.

55. O'Reilly RA. Stereoselective interaction of trimethoprimsulfamethoxazole with the separated enantiomorphs of racemic warfarin in man. $N$ Engl J Med. 1980;302(1):33-35.

56. Lewis RJ, Trager WF, Chan KK, et al. Warfarin. Stereochemical aspects of its metabolism and the interaction with phenylbutazone. J Clin Invest. 1974;53(6):1607-1617.

57. O’Reilly RA, Trager WF, Rettie AE, Goulart DA. Interaction of amiodarone with racemic warfarin and its separated enantiomorphs in humans. Clin Pharmacol Ther. 1987;42(3): 290-294.

58. Cropp JS, Bussey HI. A review of enzyme induction of warfarin metabolism with recommendations for patient management. Pharmacotherapy. 1997;17(5):917-928.

59. Ng HJ, Crowther MA. Azathioprine and inhibition of the anticoagulant effect of warfarin: evidence from a case report and a literature review. Am J Geriatr Pharmacother. 2006; 4(1):75-77.

60. O'Reilly RA. Lack of effect of fortified wine ingested during fasting and anticoagulant therapy. Arch Intern Med. 1981;141(4):458-459.

61. Bechtold H, Andrassy K, Jähnchen E, et al. Evidence for impaired hepatic vitamin K1 metabolism in patients treated with N-methyl-thiotetrazole cephalosporins. Thromb Haemost. 1984;51(3):358-361.

62. Weitekamp MR, Aber RC. Prolonged bleeding times and bleeding diathesis associated with moxalactam administration. JAMA. 1983;249(1):69-71.

63. Owens JC, Neely WB, Owen WR. Effect of sodium dextrothyroxine in patients receiving anticoagulants. $N$ Engl J Med. 1962;266:76-79.

64. O'Reilly RA, Sahud MA, Robinson AJ. Studies on the interaction of warfarin and clofibrate in man. Thromb Diath Haemorrh. 1972;27(2):309-318.

65. Rothschild BM. Hematologic perturbations associated with salicylate. Clin Pharmacol Ther. 1979;26(2):145-152.

66. Hylek EM, Heiman H, Skates SJ, Sheehan MA, Singer DE. Acetaminophen and other risk factors for excessive warfarin anticoagulation. JAMA. 1998;279(9):657-662.

67. Bell WR. Acetaminophen and warfarin: undesirable synergy. JAMA. 1998;279(9):702-703.

68. Mahé I, Bertrand N, Drouet L, et al. Interaction between paracetamol and warfarin in patients: a double-blind, placebocontrolled, randomized study. Haematologica. 2006;91(12): 1621-1627.

69. Thijssen HH, Soute BA, Vervoort LM, Claessens JG. Paracetamol (acetaminophen) warfarin interaction: NAPQI, the toxic metabolite of paracetamol, is an inhibitor of enzymes in the vitamin K cycle. Thromb Haemost. 2004;92(4): 797-802.

70. Hylek EM. Paracetamol (acetaminophen) and warfarin interaction: unraveling the pivotal role of the vitamin $\mathrm{K}$ cycle. Thromb Haemost. 2004;92(4):672-673. 
71. Weibert RT, Lorentz SM, Townsend RJ, Cook CE, Klauber MR, Jagger PI. Effect of erythromycin in patients receiving long-term warfarin therapy. Clin Pharm. 1989; $8(3): 210-214$.

72. Lorentz SM, Weibert RT. Potentiation of warfarin anticoagulation by topical testosterone ointment. Clin Pharmacol Ther. 1985;4(3):332-334.

73. Udall JA. Human sources and absorption of vitamin $\mathrm{K}$ in relation to anticoagulation stability. JAMA. 1965;194(2): 127-129.

74. Dale J, Myhre E, Loew D. Bleeding during acetylsalicylic acid and anticoagulant therapy in patients with reduced platelet reactivity after aortic valve replacement. Am Heart J. 1980;99(6):746-752.

75. Schulman S, Henriksson K. Interaction of ibuprofen and warfarin on primary haemostasis. Br I Rheumatol. 1989; 28(1):46-49.

76. Battistella M, Mamdami MM, Juurlink DN, Rabeneck L, Laupacis A. Risk of upper gastrointestinal hemorrhage in warfarin users treated with nonselective NSAIDs or COX-2 inhibitors. Arch Intern Med. 2005;165(2):189-192.

77. Cazenave JP, Packham MA, Guccione MA, Mustard JF. Effects of penicillin $\mathrm{G}$ on platelet aggregation, release, and adherence to collagen. Proc Soc Exp Biol Med. 1973;142(1): 159-166.

78. Brown CH III, Natelson EA, Bradshaw W, Williams TW Jr, Alfrey CP Jr. The hemostatic defect produced by carbenicillin. N Engl J Med. 1974;291(6):265-270

79. Roth GJ, Majerus PW. The mechanism of the effect of aspirin on human platelets. I. Acetylation of a particulate fraction protein. J Clin Invest. 1975;56(3):624-632.

80. Andreotti F, Testa L, Biondi-Zoccai GGL, Crea F. Aspirin plus warfarin compared to aspirin alone after acute coronary syndromes: an updated and comprehensive meta-analysis of 25,307 patients. Eur Heart J. 2006;27(5):519-526.

81. Chesebro JH, Fuster V, Elveback LR, et al. Trial of combined warfarin plus dipyridamole or aspirin therapy in prosthetic heart valve replacement: danger of aspirin compared with dipyridamole. Am J Cardiol. 1983;51(9):1537-1541.

82. Turpie AGG, Gent M, Laupacis A, et al. A comparison of aspirin with placebo in patients treated with warfarin after heartvalve replacement. $N$ Engl J Med. 1993;329(8):524-529.

83. Thrombosis prevention trial: randomised trial of lowintensity oral anticoagulation with warfarin and low-dose aspirin in the primary prevention of ischaemic heart disease in men at increased risk. The Medical Research Council's General Practice Research Framework. Lancet. 1998;351(9098):233-241.

84. Schelleman H, Bilker WB, Brensinger CM, Wan F, Yang YX, Hennessy S. Fibrate/Statin initiation in warfarin users and gastrointestinal bleeding risk. Am J Med. 2010;123(2): 151-157.

85. Douketis JD, Melo M, Bell CM, Mamdani MM. Does statin therapy decrease the risk for bleeding in patients who are receiving warfarin? Am J Med. 2007;120(4):369. e9-369.e14.

86. Holbrook A, Schulman S, Witt DM, et al. Evidence-based management of anticoagulant therapy: antithrombotic therapy and prevention of thrombosis, 9th ed: American College of Chest Physicians evidence-based clinical practice guidelines. Chest. 2012;141(2)(suppl 1):e152S-e184S.

87. Wittkowsky AK, Bussey HI, Walker MB, Frei CR. Dietary supplement use among anticoagulation clinic patients. J Thromb Haemost. 2007;5(4):875-877.

88. Wittkowsky AK. Drug interactions update: drugs, herbs, and oral anticoagulation. J Thromb Thrombolysis. 2001;12(1): 67-71.
89. Greenblatt DJ, von Moltke LL. Interaction of warfarin with drugs, natural substances, and foods. I Clin Pharmacol. $2005 ; 45(2): 127-132$

90. Samuels N. Herbal remedies and anticoagulant therapy. Thromb Haemost. 2005;93(1):3-7.

91. Izzo AA, Di Carlo G, Borrelli F, Ernst E. Cardiovascular pharmacotherapy and herbal medicines: the risk of drug interaction. Int J Cardiol. 2005;98(1):1-14.

92. Jiang X, Williams KM, Liauw WS, et al. Effect of ginkgo and ginger on the pharmacokinetics and pharmacodynamics of warfarin in healthy subjects. Br J Clin Pharmacol. 2005;59(4):425-432.

93. Engelsen J, Nielsen JD, Winther K. Effect of coenzyme Q10 and ginkgo biloba on warfarin dosage in stable, long-term warfarin treated outpatients: a randomized, double blind, placebocrossover trial. Thromb Haemost. 2002;87(6):1075-1076.

94. Yuan CS, Wei G, Dey L, et al. Brief communication: American ginseng reduces warfarin's effect in healthy patients: a randomized, controlled Trial. Ann Intern Med. 2004;141(1):23-27.

95. O’Reilly RA, Rytand DA. "Resistance" to warfarin due to unrecognized vitamin K supplementation. N Engl J Med. 1980;303(3):160-161.

96. Suttie JW, Mummah-Schendel LL, Shah DV, Lyle BJ, Greger JL. Vitamin K deficiency from dietary vitamin K restriction in humans. Am J Clin Nutr. 1988;47(3):475-480.

97. Sadowski JA, Booth SL, Mann KG, et al, eds. Structure and Mechanism of Activation of Vitamin K Antagonists. London, England: Arnold; 1996.

98. Bovill EG, Lawson J, Sadowski J, et al. Mechanisms of vitamin $\mathrm{K}$ metabolism and vitamin K-dependent hemostasis: Implications for warfarin therapy. In: Ezekowitz MD, ed. The Heart as a Source of Systemic Embolisation. New York, NY: Marcel Dekker; 1992.

99. Booth SL, Charnley JM, Sadowski JA, Saltzman E, Bovill EG, Cushman M. Dietary vitamin K1 and stability of oral anticoagulation: proposal of a diet with constant vitamin $\mathrm{K} 1$ content. Thromb Haemost. 1997;77(3):504-509.

100. Nutescu EA, Shapiro NL, Ibrahim S, West P. Warfarin and its interactions with foods, herbs and other dietary supplements. Expert Opin Drug Saf. 2006;5(3):433-451.

101. Harris JE. Interaction of dietary factors with oral anticoagulants: review and applications. J Am Diet Assoc. 1995; 95(5):580-584

102. Wittkowsky AK. Dietary supplements, herbs and oral anticoagulants: the nature of the evidence. I Thromb Thrombolysis. 2008;25(1):72-77.

103. Barcellona D, Contu P, Marongiu F. Patient education and oral anticoagulant therapy. Haematologica. 2002;87(10): 1081-1086.

104. Mammen EF. Coagulation abnormalities in liver disease. Hematol Oncol Clin North Am. 1992;6(6):1247-1257.

105. Deitcher SR. Interpretation of the international normalised ratio in patients with liver disease. Lancet. 2002;359(9300): 47-48.

106. Richards RK. Influence of fever upon the action of 3,3methylene bis-(4-hydroxoycoumarin). Science. 1943; 97(2518):313-316

107. Zevin S, Benowitz NL. Drug interactions with tobacco smoking. An update. Clin Pharmacokinet. 1999;36(6):425-438.

108. Evans M, Lewis GM. Increase in international normalized ratio after smoking cessation in a patient receiving warfarin. Pharmacotherapy. 2005;25(11):1656-1659.

109. Kuykendall JR, Houle MD, Rhodes RS. Possible warfarin failure due to interaction with smokeless tobacco. Ann Pharmacother. 2004;38(4):595-597.

110. Self TH, Reaves AB, Oliphant CS, Sands C. Does heart failure exacerbation increase response to warfarin? A critical 
review of the literature. Curr Med Res Opin. 2006;22(11): 2089-2094.

111. Dreisbach AW, Japa S, Gebrekal AB, et al. Cytochrome P4502C9 activity in end-stage renal disease. Clin Pharmacol Ther. 2003;73(5):475-477.

112. Gurwitz JH, Avorn J, Ross-Degnan D, Choodnovskiy I, Ansell J. Aging and the anticoagulant response to warfarin therapy. Ann Intern Med. 1992;116(11):901-904.

113. Shepherd AM, Hewick DS, Moreland TA, Stevenson IH. Age as a determinant of sensitivity to warfarin. Br J Clin Pharmacol. 1977;4(3):315-320.

114. Hodges SJ, Pilkington MJ, Shearer MJ, Bitensky L, Chayen J. Age-related changes in the circulating levels of congeners of vitamin K2, menaquinone-7 and menaquinone-8. Clin Sci (Lond). 1990;78(1):63-66.

115. Garcia D, Regan S, Crowther M, Hughes RA, Hylek EM. Warfarin maintenance dosing patterns in clinical practice: implications for safer anticoagulation in the elderly population. Chest. 2005;127(6):2049-2056.

116. Wessler S, Gitel SN. Warfarin. From bedside to bench. N Engl J Med. 1984;311(10):645-652.

117. Zivelin A, Rao LV, Rapaport SI. Mechanism of the anticoagulant effect of warfarin as evaluated in rabbits by selective depression of individual procoagulant vitamin K-dependent clotting factors. J Clin Invest. 1993;92(5):2131-2140.

118. Patel P, Weitz J, Brooker LA, Paes B, Mitchell L, Andrew M. Decreased thrombin activity of fibrin clots prepared in cord plasma compared with adult plasma. Pediatr Res. 1996; 39(5):826-830.

119. Weitz JI, Hudoba M, Massel D, Maraganore J, Hirsh J. Clot-bound thrombin is protected from inhibition by heparin-antithrombin III but is susceptible to inactivation by antithrombin III-independent inhibitors. J Clin Invest. 1990;86(2):385-391.

120. Quick A. The prothrombin time in haemophilia and in obstructive jaundice. J Biol Chem. 1935;109:73-74.

121. Kirkwood TB. Calibration of reference thromboplastins and standardisation of the prothrombin time ratio. Thromb Haemost. 1983;49(3):238-244.

122. van den Besselaar AM, Chantarangkul V, Tripodi A. Thromboplastin standards. Biologicals. 2010;38(4):430-436.

123. World Health Organization Expert Committee on Biological Standardization. 33rd Report: Technical Report Series No. 687. Geneva, Switzerland: World Health Organization. 1983

124. Johnston M, Harrison L, Moffat K, Willan A, Hirsh J. Reliability of the international normalized ratio for monitoring the induction phase of warfarin: comparison with the prothrombin time ratio. J Lab Clin Med. 1996;128(2):214-217.

125. Tripodi A, Chantarangkul V, Primignani M, et al. The international normalized ratio calibrated for cirrhosis (INR(liver)) normalizes prothrombin time results for model for end-stage liver disease calculation. Hepatology. 2007; 46(2):520-527.

126. Bellest L, Eschwège $\mathrm{V}$, Poupon $\mathrm{R}$, Chazouillères $\mathrm{O}$, Robert $\mathrm{A}$. A modified international normalized ratio as an effective way of prothrombin time standardization in hepatology. Hepatology. 2007;46(2):528-534.

127. Lind SE, Pearce LA, Feinberg WM, Bovill EG. Clinically significant differences in the International Normalized Ratio measured with reagents of different sensitivities. SPAF Investigators. Stroke Prevention in Atrial Fibrillation. Blood Coagul Fibrinolysis. 1999;10(5):215-227.

128. Poggio M, van den Besselaar AMHP, van der Velde EA, Bertina RM. The effect of some instruments for prothrombin time testing on the International Sensitivity Index (ISI) of two rabbit tissue thromboplastin reagents. Thromb Haemost. 1989;62(3):868-874.
129. D’Angelo A, Seveso MP, D’Angelo SV, et al. Comparison of two automated coagulometers and the manual tilt-tube method for the determination of prothrombin time. Am J Clin Pathol. 1989;92(3):321-328.

130. Poller L, Thomson JM, Taberner DA. Effect of automation on prothrombin time test in NEQAS surveys. J Clin Pathol. 1989;42(1):97-100.

131. Ray MJ, Smith IR. The dependence of the International Sensitivity Index on the coagulometer used to perform the prothrombin time. Thromb Haemost. 1990;63(3):424-429.

132. van Rijn JLML, Schmidt NA, Rutten W. Correction of instrument and reagent based differences in determination of the international normalised ratio (INR) for monitoring anticoagulant therapy. Clin Chem. 1989;35(5):840-843.

133. Thomson JM, Taberner DA, Poller L. Automation and prothrombin time: a United Kingdom field study of two widely used coagulometers. J Clin Pathol. 1990;43(8): 679-684.

134. Finck KM, Doetkott C, Miller DR. Clinical impact of interlaboratory variation in international normalized ratio determinations. Am J Health Syst Pharm. 2001;58(8):684-688.

135. Jacobson AK, Ruybalid RL, Johnston M, et al. Significant variation in the reporting of prothrombin time results despite utilization of the INR method of reporting [abstract]. Circulation. 1999;100:I-620.

136. Fairweather RB, Ansell J, van den Besselaar AM, et al. College of American Pathologists Conference XXXI on laboratory monitoring of anticoagulant therapy: laboratory monitoring of oral anticoagulant therapy. Arch Pathol Lab Med. 1998;122(9):768-781.

137. Ng VL, Levin J, Corash L, Gottfried EL. Failure of the International Normalized Ratio to generate consistent results within a local medical community. Am J Clin Pathol. 1993; 99(6):689-694.

138. Poller L. Laboratory control of oral anticoagulants. Br Med J (Clin Res Ed). 1987;294(6581):1184.

139. Kazama M, Suzuki S, Abe T, et al. Evaluation of international normalized ratios by a controlled field survey with 4 different thromboplastin reagents. Thromb Haemost. 1990; 64(4):535-541.

140. van den Besselaar AM, Lewis SM, Mannucci PM, Poller L. Status of present and candidate International Reference Preparations (IRP) of thromboplastin for the prothrombin time: a report of the Subcommittee for Control of Anticoagulation. Thromb Haemost. 1993;69(1):85.

141. Duncan EM, Casey CR, Duncan BM, Lloyd JV. Effect of concentration of trisodium citrate anticoagulant on calculation of the International Normalised Ratio and the International Sensitivity Index of thromboplastin. Thromb Haemost. 1994;72(1):84-88.

142. Adcock DM, Kressin DC, Marlar RA. Effect of $3.2 \%$ vs $3.8 \%$ sodium citrate concentration on routine coagulation testing. Am J Clin Pathol. 1997;107(1):105-110.

143. Harrison L, Johnston M, Massicotte MP, Crowther M, Moffat K, Hirsh J. Comparison of 5-mg and 10-mg loading doses in initiation of warfarin therapy. Ann Intern Med. 1997;126(2):133-136.

144. O’Reilly RA, Aggeler PM. Studies on coumarin anticoagulant drugs. Initiation of warfarin therapy without a loading dose. Circulation. 1968;38(1):169-177.

145. Crowther MA, Ginsberg JB, Kearon C, et al. A randomized trial comparing 5-mg and 10-mg warfarin loading doses. Arch Intern Med. 1999;159(1):46-48.

146. Kovacs MJ, Rodger M, Anderson DR, et al. Comparison of $10-\mathrm{mg}$ and $5-\mathrm{mg}$ warfarin initiation nomograms together with low-molecular-weight heparin for outpatient treatment of acute venous thromboembolism. A randomized, 
double-blind, controlled trial. Ann Intern Med. 2003;138(9): 714-719.

147. Siguret V, Gouin I, Debray M, et al. Initiation of warfarin therapy in elderly medical inpatients: a safe and accurate regimen. Am J Med. 2005;118(2):137-142.

148. O’Connell MB, Kowal PR, Allivato CJ, Repka TL. Evaluation of warfarin initiation regimens in elderly inpatients. Pharmacotherapy. 2000;20(8):923-930.

149. Ageno W, Turpie AGG, Steidl L, et al. Comparison of a daily fixed 2.5-mg warfarin dose with a 5-mg, international normalized ratio adjusted, warfarin dose initially following heart valve replacement. Am J Cardiol. 2001;88(1):40-44.

150. Vecsler M, Loebstein R, Almog S, et al. Combined genetic profiles of components and regulators of the vitamin K-dependent gamma-carboxylation system affect individual sensitivity to warfarin. Thromb Haemost. 2006;95(2):205-211.

151. Herman D, Peternel P, Stegnar M, Breskvar K, Dolzan V. The influence of sequence variations in factor VII, gammaglutamyl carboxylase and vitamin $\mathrm{K}$ epoxide reductase complex genes on warfarin dose requirement. Thromb Haemost. 2006;95(5):782-787.

152. Limdi NA, Arnett DK, Goldstein JA, et al. Influence of CYP2C9 and VKORC1on warfarin dose, anticoagulation attainment and maintenance among European-Americans and African-Americans. Pharmacogenomics. 2008;9(5):511-526.

153. Voora D, Eby C, Linder MW, et al. Prospective dosing of warfarin based on cytochrome P-450 2C9 genotype. Thromb Haemost. 2005;93(4):700-705.

154. Gage BF, Eby C, Johnson JA, et al. Use of pharmacogenetic and clinical factors to predict the therapeutic dose of warfarin. Clin Pharmacol Ther. 2008;84(3):326-331.

155. Anderson JL, Horne BD, Stevens SM, et al; Couma-Gen Investigators. Randomized trial of genotype-guided versus standard warfarin dosing in patients initiating oral anticoagulation. Circulation. 2007;116(22):2563-2570.

156. Caraco Y, Blotnick S, Muszkat M. CYP2C9 genotype-guided warfarin prescribing enhances the efficacy and safety of anticoagulation: a prospective randomized controlled study. Clin Pharmacol Ther. 2008;83(3):460-470.

157. Hillman MA, Wilke RA, Yale SH, et al. A prospective, randomized pilot trial of model-based warfarin dose initiation using CYP2C9 genotype and clinical data. Clin Med Res. 2005;3(3):137-145.

158. Klein TE, Altman RB, Eriksson N, et al; International Warfarin Pharmacogenetics Consortium. Estimation of the warfarin dose with clinical and pharmacogenetic data [published correction appears in N Engl J Med 2009;361(16):1613]. N Engl J Med. 2009;360(8):753-764.

159. Joffe $\mathrm{HV}, \mathrm{Xu} \mathrm{R}$, Johnson FB, Longtine J, Kucher N, Goldhaber SZ. Warfarin dosing and cytochrome P450 2C9 polymorphisms. Thromb Haemost. 2004;91(6):1123-1128.

160. Limdi NA, Wiener H, Goldstein JA, Acton RT, Beasley TM. Influence of CYP2C9 and VKORC1 on warfarin response during initiation of therapy. Blood Cells $\mathrm{Mol}$ Dis. 2009;43(1):119-128.

161. Palareti G, Legnani C, Guazzaloca G, et al; ad hoc Study Group of the Italian Federation of Anticoagulation Clinics*. Risks factors for highly unstable response to oral anticoagulation: a case-control study. Br J Haematol. 2005;129(1): $72-78$.

162. Forfar JC. Prediction of hemorrhage during long-term oral coumarin anticoagulation by excessive prothrombin ratio. Am Heart J. 1982;103(3):445-446.

163. White HD, Gruber M, Feyzi J, et al. Comparison of outcomes among patients randomized to warfarin therapy according to anticoagulant control: results from SPORTIF III and V. Arch Intern Med. 2007;167(3):239-245.
164. Cannegieter SC, Rosendaal FR, Wintzen AR, van der Meer FJ, Vandenbroucke JP, Briët E. Optimal oral anticoagulant therapy in patients with mechanical heart valves. $N$ Engl J Med. 1995;333(1):11-17.

165. Connolly SJ, Pogue J, Eikelboom J, et al. Benefit of oral anticoagulant over antiplatelet therapy in atrial fibrillation depends on the quality of international normalized ratio control achieved by centers and countries as measured by time in therapeutic range. Circulation. 2008;118(20):2029-2037.

166. Petersen P, Boysen G, Godtfredsen J, Andersen ED, Andersen B. Placebo-controlled, randomised trial of warfarin and aspirin for prevention of thromboembolic complications in chronic atrial fibrillation. The Copenhagen AFASAK study. Lancet. 1989;1(8631):175-179.

167. The Boston Area Anticoagulation Trial for Atrial Fibrillation Investigators. The effect of low-dose warfarin on the risk of stroke in patients with nonrheumatic atrial fibrillation. N Engl J Med. 1990;323(22):1505-1511.

168. Ezekowitz MD, Bridgers SL, James KE, et al; Veterans Affairs Stroke Prevention in Nonrheumatic Atrial Fibrillation Investigators. Warfarin in the prevention of stroke associated with nonrheumatic atrial fibrillation. $N$ Engl J Med. 1992;327(20):1406-1412.

169. The European Atrial Fibrillation Trial Study Group. Optimal oral anticoagulant therapy in patients with nonrheumatic atrial fibrillation and recent cerebral ischemia. $N$ Engl J Med. 1995;333(1):5-10.

170. van der Meer FJM, Rosendaal FR, Vandenbroucke JP, Briët E. Bleeding complications in oral anticoagulant therapy. An analysis of risk factors. Arch Intern Med. 1993; 153(13):1557-1562.

171. Charney R, Leddomado E, Rose DN, Fuster V. Anticoagulation clinics and the monitoring of anticoagulant therapy. Int J Cardiol. 1988;18(2):197-206.

172. Wilson DB, Dunn MI, Hassanein K. Low-intensity anticoagulation in mechanical cardiac prosthetic valves. Chest. 1991;100(6):1553-1557.

173. Connolly SJ, Laupacis A, Gent M, Roberts RS, Cairns JA, Joyner C. Canadian atrial fibrillation anticoagulation (CAFA) study. J Am Coll Cardiol. 1991;18(2):349-355.

174. Hylek EM, Skates SJ, Sheehan MA, Singer DE. An analysis of the lowest effective intensity of prophylactic anticoagulation for patients with nonrheumatic atrial fibrillation. $N$ Engl J Med. 1996;335(8):540-546.

175. Palareti G, Legnani C, Cosmi B, Guazzaloca G, Cini M, Mattarozzi S. Poor anticoagulation quality in the first 3 months after unprovoked venous thromboembolism is a risk factor for long-term recurrence. J Thromb Haemost. 2005;3(5): 955-961.

176. Schmitt L, Speckman J, Ansell J. Quality assessment of anticoagulation dose management: comparative evaluation of measures of time-in-therapeutic range. J Thromb Thrombolysis. 2003;15(3):213-216.

177. Rosendaal FR, Cannegieter SC, van der Meer FJ, Briët E. A method to determine the optimal intensity of oral anticoagulant therapy. Thromb Haemost. 1993;69(3):236-239.

178. Meier DJ, Seva S, Fay WP. A comparison of anticoagulation results of patients managed with narrow vs. standard international normalized ratio target ranges. J Thromb Haemost. 2007;5(6):1332-1334.

179. Gadisseur AP, van der Meer FJM, Adriaansen HJ, Fihn SD, Rosendaal FR. Therapeutic quality control of oral anticoagulant therapy comparing the short-acting acenocoumarol and the long-acting phenprocoumon. Br J Haematol. 2002;117(4):940-946.

180. Pattacini C, Manotti C, Pini M, Quintavalla R, Dettori AG. A comparative study on the quality of oral anticoagulant 
therapy (warfarin versus acenocoumarol). Thromb Haemost. 1994;71(2):188-191.

181. Redwood M, Taylor C, Bain BJ, Matthews JH. The association of age with dosage requirement for warfarin. Age Ageing. 1991;20(3):217-220.

182. James AH, Britt RP, Raskino CL, Thompson SG. Factors affecting the maintenance dose of warfarin. J Clin Pathol. 1992;45(8):704-706.

183. Mungall D, White R. Aging and warfarin therapy. Ann Intern Med. 1992;117(10):878-879.

184. Pengo V, Barbero F, Biasiolo A, Pegoraro C, Cucchini U, Iliceto S. A comparison between six- and four-week intervals in surveillance of oral anticoagulant treatment. Am J Clin Pathol. 2003;120(6):944-947.

185. Shalev V, Rogowski O, Shimron $\mathrm{O}$, et al. The interval between prothrombin time tests and the quality of oral anticoagulants treatment in patients with chronic atrial fibrillation. Thromb Res. 2007;120(2):201-206.

186. Witt DM, Delate T, Clark NP, et al; Warfarin Associated Research Projects and other EnDeavors (WARPED) Consortium. Outcomes and predictors of very stable INR control during chronic anticoagulation therapy. Blood. 2009; 114(5):952-956

187. Witt DM, Delate T, Clark NP, et al; Warped Consortium. Twelve-month outcomes and predictors of very stable INR control in prevalent warfarin users. J Thromb Haemost. 2010; 8(4):744-749.

188. Shibata Y, Hashimoto H, Kurata C, Ohno R, Kazui T, Takinami M. Influence of physical activity on warfarin therapy. Thromb Haemost. 1998;80(1):203-204.

189. Lenz TL, Lenz NJ, Faulkner MA. Potential interactions between exercise and drug therapy. Sports Med. 2004;34(5): 293-306.

190. Sorano GG, Biondi G, Conti M, Mameli G, Licheri D, Marongiu F. Controlled vitamin K content diet for improving the management of poorly controlled anticoagulated patients: a clinical practice proposal. Haemostasis. 1993; 23(2):77-82.

191. Sconce E, Khan T, Mason J, Noble F, Wynne H, Kamali F. Patients with unstable control have a poorer dietary intake of vitamin $\mathrm{K}$ compared to patients with stable control of anticoagulation. Thromb Haemost. 2005;93(5):872-875.

192. Kurnik D, Loebstein R, Rabinovitz H, Austerweil N, Halkin H, Almog S. Over-the-counter vitamin K1-containing multivitamin supplements disrupt warfarin anticoagulation in vitamin K1-depleted patients. A prospective, controlled trial. Thromb Haemost. 2004;92(5):1018-1024.

193. Schurgers LJ, Shearer MJ, Hamulyák K, Stöcklin E, Vermeer C. Effect of vitamin K intake on the stability of oral anticoagulant treatment: dose-response relationships in healthy subjects. Blood. 2004;104(9):2682-2689.

194. Reese AM, Farnett LE, Lyons RM, Patel B, Morgan L, Bussey HI. Low-dose vitamin $\mathrm{K}$ to augment anticoagulation control. Pharmacotherapy. 2005;25(12):1746-1751.

195. Ford SK, Misita CP, Shilliday BB, Malone RM, Moore CG, Moll S. Prospective study of supplemental vitamin K therapy in patients on oral anticoagulants with unstable international normalized ratios. J Thromb Thrombolysis. 2007;24(1): 23-27.

196. Rombouts EK, Rosendaal FR, van der Meer FJ. Influence of dietary vitamin $\mathrm{K}$ intake on subtherapeutic oral anticoagulant therapy. Br J Haematol. 2010;149(4):598-605.

197. Sconce E, Avery P, Wynne H, Kamali F. Vitamin K supplementation can improve stability of anticoagulation for patients with unexplained variability in response to warfarin. Blood. 2007;109(6):2419-2423.
198. Rombouts EK, Rosendaal FR, Van Der Meer FJ. Daily vitamin $\mathrm{K}$ supplementation improves anticoagulant stability. J Thromb Haemost. 2007;5(10):2043-2048.

199. de Assis MC, Rabelo ER, Avila CW, Polanczyk CA, Rohde LE. Improved oral anticoagulation after a dietary vitamin k-guided strategy: a randomized controlled trial. Circulation. 2009;120(12):1115-1122.

200. Kimmel SE, Chen Z, Price M, et al. The influence of patient adherence on anticoagulation control with warfarin: results from the International Normalized Ratio Adherence and Genetics (IN-RANGE) Study. Arch Intern Med. 2007; 167(3):229-235.

201. Orensky IA, Holdford DA. Predictors of noncompliance with warfarin therapy in an outpatient anticoagulation clinic. Pharmacotherapy. 2005;25(12):1801-1808.

202. Platt AB, Localio AR, Brensinger CM, et al. Risk factors for nonadherence to warfarin: results from the IN-RANGE study. Pharmacoepidemiol Drug Saf. 2008;17(9):853-860.

203. Beyth RJ, Quinn LM, Landefeld CS. Prospective evaluation of an index for predicting the risk of major bleeding in outpatients treated with warfarin. Am J Med. 1998;105(2):91-99.

204. Gitter MJ, Jaeger TM, Petterson TM, Gersh BJ, Silverstein MD. Bleeding and thromboembolism during anticoagulant therapy: a population-based study in Rochester, Minnesota. Mayo Clin Proc. 1995;70(8):725-733.

205. Steffensen FH, Kristensen K, Ejlersen E, Dahlerup JF, Sørensen HT. Major haemorrhagic complications during oral anticoagulant therapy in a Danish population-based cohort. J Intern Med. 1997;242(6):497-503.

206. Willey VJ, Bullano MF, Hauch O, et al. Management patterns and outcomes of patients with venous thromboembolism in the usual community practice setting. Clin Ther. 2004;26(7):1149-1159.

207. Palareti G, Leali N, Coccheri S, et al; Italian Study on Complications of Oral Anticoagulant Therapy. Bleeding complications of oral anticoagulant treatment: an inceptioncohort, prospective collaborative study (ISCOAT). Lancet. 1996;348(9025):423-428.

208. Palareti G, Manotti C, DAngelo A, et al. Thrombotic events during oral anticoagulant treatment: results of the inceptioncohort, prospective, collaborative ISCOAT study: ISCOAT study group (Italian Study on Complications of Oral Anticoagulant Therapy). Thromb Haemost. 1997;78(6):1438-1443.

209. Abdelhafiz AH, Wheeldon NM. Results of an open-label, prospective study of anticoagulant therapy for atrial fibrillation in an outpatient anticoagulation clinic. Clin Ther. 2004;26(9):1470-1478.

210. Veeger NJGM, Piersma-Wichers M, Tijssen JGP, Hillege HL, van der Meer J. Individual time within target range in patients treated with vitamin $\mathrm{K}$ antagonists: main determinant of quality of anticoagulation and predictor of clinical outcome. A retrospective study of 2300 consecutive patients with venous thromboembolism. Br J Haematol. 2005;128(4):513-519.

211. Cortelazzo S, Finazzi G, Viero P, et al. Thrombotic and hemorrhagic complications in patients with mechanical heart valve prosthesis attending an anticoagulation clinic. Thromb Haemost. 1993;69(4):316-320.

212. Chiquette E, Amato MG, Bussey HI. Comparison of an anticoagulation clinic with usual medical care: anticoagulation control, patient outcomes, and health care costs. Arch Intern Med. 1998;158(15):1641-1647.

213. Witt DM, Sadler MA, Shanahan RL, Mazzoli G, Tillman DJ. Effect of a centralized clinical pharmacy anticoagulation service on the outcomes of anticoagulation therapy. Chest. 2005;127(5):1515-1522. 
214. Matchar DB, Samsa GP, Cohen SJ, Oddone EZ, Jurgelski AE. Improving the quality of anticoagulation of patients with atrial fibrillation in managed care organizations: results of the managing anticoagulation services trial. Am J Med. 2002;113(1):42-51.

215. Wilson SJ, Wells PS, Kovacs MJ, et al. Comparing the quality of oral anticoagulant management by anticoagulation clinics and by family physicians: a randomized controlled trial. CMAJ. 2003;169(4):293-298.

216. Ansell J, Hollowell J, Pengo V, Martinez-Brotons F, Caro J, Drouet L. Descriptive analysis of the process and quality of oral anticoagulation management in real-life practice in patients with chronic non-valvular atrial fibrillation: the international study of anticoagulation management (ISAM). J Thromb Thrombolysis. 2007;23(2):83-91.

217. van Walraven C, Jennings A, Oake N, Fergusson D, Forster AJ. Effect of study setting on anticoagulation control: a systematic review and metaregression. Chest. 2006;129(5):1155-1166.

218. Wilson R, James AH. Computer assisted management of warfarin treatment. Br Med J (Clin Res Ed). 1984;289(6442): 422-424.

219. Ryan PJ, Gilbert M, Rose PE. Computer control of anticoagulant dose for therapeutic management. BMJ. 1989; 299(6709):1207-1209.

220. Weston Smith SG, Savidge GF. Computer control of anticoagulant dose. BMJ. 1989;299:1529.

221. Poller L, Wright D, Rowlands M. Prospective comparative study of computer programs used for management of warfarin. J Clin Pathol. 1993;46(4):299-303.

222. Ageno W, Turpie AGG, Steidl L, et al. A randomized comparison of a computer-based dosing program with a manual system to monitor oral anticoagulant therapy. Thromb Res. 1998;91(5):237-240.

223. Poller L, Shiach CR, MacCallum P, et al. Multicentre randomized study of computerized anticoagulant dosage. European Concerted Action on Anticoagulation. Lancet. 1998;352(9139):1505-1509.

224. Manotti C, Moia M, Palareti G, Pengo V, Ria L, Dettori AG. Effect of computer-aided management on the quality of treatment in anticoagulated patients: a prospective, randomized, multicenter trial of APROAT (Automated PRogram for Oral Anticoagulant Treatment). Haematologica. 2001;86(10): 1060-1070.

225. Poller L, Keown M, Ibrahim S, et al. An international multicenter randomized study of computer-assisted oral anticoagulant dosage vs. medical staff dosage. J Thromb Haemost. 2008;6(6):935-943.

226. Jowett S, Bryan S, Poller L, et al. The cost-effectiveness of computer-assisted anticoagulant dosage: results from the European Action on Anticoagulation (EAA) multicentre study. J Thromb Haemost. 2009;7(9):1482-1490.

227. Ageno W, Johnson J, Nowacki B, Turpie AG. A computer generated induction system for hospitalized patients starting on oral anticoagulant therapy. Thromb Haemost. 2000; 83(6):849-852.

228. Ansell JE, Leaning KE, eds. Capillary Whole Blood Prothrombin Time Monitoring: Instrumentation and Methodologies. New York, NY: Aspen; 1997.

229. Lucas FV, Duncan A, Jay R, et al. A novel whole blood capillary technic for measuring the prothrombin time. Am J Clin Pathol. 1987;88(4):442-446.

230. Yano Y, Kambayashi J, Murata K, et al. Bedside monitoring of warfarin therapy by a whole blood capillary coagulation monitor. Thromb Res. 1992;66(5):583-590.

231. Weibert RT, Adler DS. Evaluation of a capillary wholeblood prothrombin time measurement system. Clin Pharm. 1989;8(12):864-867.
232. Rose VL, Dermott SC, Murray BF, McIver MM, High KA, Oberhardt BJ. Decentralized testing for prothrombin time and activated partial thromboplastin time using a dry chemistry portable analyzer. Arch Pathol Lab Med. 1993; 117(6):611-617.

233. Gosselin R, Owings JT, White RH, et al. A comparison of point-of-care instruments designed for monitoring oral anticoagulation with standard laboratory methods. Thromb Haemost. 2000;83(5):698-703.

234. van den Besselaar AM. A comparison of INRs determined with a whole blood prothrombin time device and two international reference preparations for thromboplastin. Thromb Haemost. 2000;84(3):410-412.

235. Kitchen S, Preston FE. Monitoring oral anticoagulant treatment with the TAS near-patient test system: comparison with conventional thromboplastins. J Clin Pathol. 1997;50(11): 951-956.

236. Douketis JD, Lane A, Milne J, Ginsberg JS. Accuracy of a portable International Normalization Ratio monitor in outpatients receiving long-term oral anticoagulant therapy: comparison with a laboratory reference standard using clinically relevant criteria for agreement. Thromb Res. 1998; 92(1):11-17.

237. Cosmi B, Palareti G, Moia M, et al. Accuracy of a portable prothrombin time monitor (Coagucheck) in patients on chronic oral anticoagulant therapy: a prospective multicenter study. Thromb Res. 2000;100(4):279-286.

238. Oral Anticoagulation Monitoring Study Group. Prothrombin measurement using a patient self-testing system. Am J Clin Pathol. 2001;115(2):280-287.

239. Oral Anticoagulation Monitoring Study Group. Point-of-care prothrombin time measurement for professional and patient self-testing use. A multicenter clinical experience. Am J Clin Pathol. 2001;115(2):288-296.

240. Cachia PG, McGregor E, Adlakha S, Davey P, Goudie BM. Accuracy and precision of the TAS analyser for near-patient INR testing by non-pathology staff in the community. J Clin Pathol. 1998;51(1):68-72.

241. Murray ET, Greaves M. INRs and point of care testing. BMJ. 2003;327(7405):5-6.

242. Jennings I, Luddington RJ, Baglin T. Evaluation of the Ciba Corning Biotrack 512 coagulation monitor for the control of oral anticoagulation. J Clin Pathol. 1991;44(11): 950-953.

243. McCurdy SA, White RH. Accuracy and precision of a portable anticoagulation monitor in a clinical setting. Arch Intern Med. 1992;152(3):589-592.

244. Tripodi A, Arbini AA, Chantarangkul V, Bettega D, Mannucci PM. Are capillary whole blood coagulation monitors suitable for the control of oral anticoagulant treatment by the international normalized ratio? Thromb Haemost. 1993; 70(6):921-924.

245. Plesch W, Wolf T, Breitenbeck N, et al. Results of the performance verification of the CoaguChek XS system. Thromb Res. 2008;123(2):381-389.

246. Ryan F, O'Shea S, Byrne S. The reliability of point-of-care prothrombin time testing. A comparison of CoaguChek S and XS INR measurements with hospital laboratory monitoring. Int J Lab Hematol. 2010;32(1 pt 1):e26-e33.

247. Plesch W, van den Besselaar AM. Validation of the international normalized ratio (INR) in a new point-of-care system designed for home monitoring of oral anticoagulation therapy. Int J Lab Hematol. 2009;31(1):20-25.

248. Yelland LN, Gialamas A, Laurence CO, Willson KJ, Ryan P, Beilby JJ; PoCT Trial Management Committee. Assessing agreement between point of care and pathology laboratory results for INR: experiences from the Point of Care 
Testing in General Practice Trial. Pathology. 2010;42(2): 155-159.

249. Tripodi A, Chantarangkul V, Clerici M, Negri B, Mannucci PM. Determination of the International Sensitivity Index of a new near-patient testing device to monitor oral anticoagulant therapy-overview of the assessment of conformity to the calibration model. Thromb Haemost. 1997;78(2):855-858.

250. Perry SL, Samsa GP, Ortel TL. Point-of-care testing of the international normalized ratio in patients with antiphospholipid antibodies. Thromb Haemost. 2005;94(6):1196-1202.

251. Gialamas A, St John A, Laurence CO, Bubner TK; PoCT Management Committee. Point-of-care testing for patients with diabetes, hyperlipidaemia or coagulation disorders in the general practice setting: a systematic review. Fam Pract. 2010;27(1):17-24.

252. Kaatz SS, White RH, Hill J, Mascha E, Humphries JE, Becker DM. Accuracy of laboratory and portable monitor international normalized ratio determinations. Comparison with a criterion standard. Arch Intern Med. 1995;155(17): 1861-1867.

253. Poller L, Keown M, Chauhan N, et al. European Concerted Action on Anticoagulation (ECAA): multicentre international sensitivity index calibration of two types of point-ofcare prothrombin time monitor systems. Br J Haematol. 2002;116(4):844-850.

254. Poller L, Keown M, Chauhan N, et al. European Concerted Action on Anticoagulation (ECAA). An assessment of lyophilised plasmas for ISI calibration of CoaguChek and TAS whole blood prothrombin time monitors. J Clin Pathol. 2003;56(2):114-119.

255. Poller L, Keown M, Chauhan N, et al. Reliability of international normalised ratios from two point of care test systems: comparison with conventional methods. BMJ. 2003; 327(7405):30-34.

256. Kitchen S, Kitchen DP, Jennings I, Woods TAL, Walker ID, Preston FE. Point-of-care International Normalised Ratios: UK NEQAS experience demonstrates necessity for proficiency testing of three different monitors. Thromb Haemost. 2006;96(5):590-596.

257. Jespersen J, Poller L, van den Besselaar AM, et al. External quality assessment (EQA) for CoaguChek monitors. Thromb Haemost. 2010;103(5):936-941.

258. Ansell JE, Hughes R. Evolving models of warfarin management: anticoagulation clinics, patient self-monitoring, and patient self-management. Am Heart J. 1996;132(5):1095-1100.

259. Christensen TD. Self-management of oral anticoagulant therapy: a review. J Thromb Thrombolysis. 2004;18(2):127-143.

260. Siebenhofer A, Berghold A, Sawicki PT. Systematic review of studies of self-management of oral anticoagulation. Thromb Haemost. 2004;91(2):225-232.

261. Heneghan C, Alonso-Coello P, Garcia-Alamino JM, Perera R, Meats E, Glasziou P. Self-monitoring of oral anticoagulation: a systematic review and meta-analysis. Lancet. 2006; 367(9508):404-411.

262. Dauphin C, Legault B, Jaffeux P, et al. Comparison of INR stability between self-monitoring and standard laboratory method: preliminary results of a prospective study in 67 mechanical heart valve patients. Arch Cardiovasc Dis. 2008; 101(11-12):753-761.

263. Eitz T, Schenk S, Fritzsche D, et al. International normalized ratio self-management lowers the risk of thromboembolic events after prosthetic heart valve replacement. Ann Thorac Surg. 2008;85(3):949-954.

264. Ansell J, Jacobson A, Levy J, Völler H, Hasenkam JM; International Self-Monitoring Association for Oral Anticoagulation. Guidelines for implementation of patient self- testing and patient self-management of oral anticoagulation. International consensus guidelines prepared by International Self-Monitoring Association for Oral Anticoagulation. Int J Cardiol. 2005;99(1):37-45

265. Fitzmaurice DA, Gardiner C, Kitchen S, Mackie I, Murray ET, Machin SJ; British Society of Haematology Taskforce for Haemostasis and Thrombosis. An evidencebased review and guidelines for patient self-testing and management of oral anticoagulation. Br J Haematol. 2005; 131(2):156-165

266. Landefeld CS, Rosenblatt MW, Goldman L. Bleeding in outpatients treated with warfarin: relation to the prothrombin time and important remediable lesions. Am J Med. 1989; $87(2): 153-159$

267. Fihn SD, McDonell M, Martin D, et al. Risk factors for complications of chronic anticoagulation. A multi-center study. Warfarin Optimized Outpatient Follow-up Study Group. Ann Intern Med. 1993;118(7):511-520.

268. Hylek EM, Singer DE. Risk factors for intracranial hemorrhage in outpatients taking warfarin. Ann Intern Med. 1994;120(11):897-902.

269. Hull R, Hirsh J, Jay R, et al. Different intensities of oral anticoagulant therapy in the treatment of proximal-vein thrombosis. N Engl J Med. 1982;307(27):1676-1681.

270. Ridker PM, Goldhaber SZ, Danielson E, et al; PREVENT Investigators. Long-term, low-intensity warfarin therapy for the prevention of recurrent venous thromboembolism. N Engl J Med. 2003;348(15):1425-1434.

271. Kearon C, Ginsberg JS, Kovacs MJ, et al; Extended LowIntensity Anticoagulation for Thrombo-Embolism Investigators. Comparison of low-intensity warfarin therapy with conventional-intensity warfarin therapy for long-term prevention of recurrent venous thromboembolism. $N$ Engl J Med. 2003;349(7):631-639.

272. Turpie AGG, Gunstensen J, Hirsh J, Nelson H, Gent M. Randomised comparison of two intensities of oral anticoagulant therapy after tissue heart valve replacement. Lancet. 1988;1(8597):1242-1245.

273. Saour JN, Sieck JO, Mamo LAR, Gallus AS. Trial of different intensities of anticoagulation in patients with prosthetic heart valves. N Engl J Med. 1990;322(7):428-432.

274. Altman R, Rouvier J, Gurfinkel E, et al. Comparison of two levels of anticoagulant therapy in patients with substitute heart valves. J Thorac Cardiovasc Surg. 1991;101(3):427-431.

275. Hering D, Piper C, Bergemann R, et al. Thromboembolic and bleeding complications following St. Jude Medical valve replacement: results of the German Experience With LowIntensity Anticoagulation Study. Chest. 2005;127(1):53-59.

276. Vink R, Kraaijenhagen RA, Hutten BA, et al. The optimal intensity of vitamin $\mathrm{K}$ antagonists in patients with mechanical heart valves: a meta-analysis. J Am Coll Cardiol. 2003; 42(12):2042-2048.

277. Anand SS, Yusuf S. Oral anticoagulant therapy in patients with coronary artery disease: a meta-analysis. JAMA. 1999; 282(21):2058-2067.

278. Segal JB, McNamara RL, Miller MR, et al. Prevention of thromboembolism in atrial fibrillation. A meta-analysis of trials of anticoagulants and antiplatelet drugs. J Gen Intern Med. 2000;15(1):56-67.

279. Hart RG, Benavente O, McBride R, Pearce LA. Antithrombotic therapy to prevent stroke in patients with atrial fibrillation: a meta-analysis. Ann Intern Med. 1999; 131(7):492-501.

280. Risk factors for stroke and efficacy of antithrombotic therapy in atrial fibrillation. Analysis of pooled data from five randomized controlled trials. Arch Intern Med. 1994;154(13): 1449-1457. 
281. The Stroke Prevention in Reversible Ischemia Trial (SPIRIT) Study Group. A randomized trial of anticoagulants versus aspirin after cerebral ischemia of presumed arterial origin. Ann Neurol. 1997;42(6):857-865.

282. Stroke Prevention in Atrial Fibrillation III Investigators. Adjusted-dose warfarin versus low-intensity, fixed-dose warfarin plus aspirin for high-risk patients with atrial fibrillation: Stroke Prevention in Atrial Fibrillation III randomised clinical trial. Lancet. 1996;348(9028):633-638.

283. Hylek EM, Go AS, Chang Y, et al. Effect of intensity of oral anticoagulation on stroke severity and mortality in atrial fibrillation. N Engl J Med. 2003;349(11):1019-1026.

284. Indredavik B, Rohweder G, Lydersen S. Frequency and effect of optimal anticoagulation before onset of ischaemic stroke in patients with known atrial fibrillation. J Intern Med. 2005;258(2): 133-144.

285. O'Donnell M, Oczkowski W, Fang J, et al; Investigators of the Registry of the Canadian Stroke Network. Preadmission antithrombotic treatment and stroke severity in patients with atrial fibrillation and acute ischaemic stroke: an observational study. Lancet Neurol. 2006;5(9):749-754.

286. Crowther MA, Clase CM, Margetts PJ, et al. Low-intensity warfarin is ineffective for the prevention of PTFE graft failure in patients on hemodialysis: a randomized controlled trial. J Am Soc Nephrol. 2002;13(9):2331-2337.

287. Gulløv AL, Koefoed BG, Petersen P, et al. Fixed minidose warfarin and aspirin alone and in combination vs adjusteddose warfarin for stroke prevention in atrial fibrillation: Second Copenhagen Atrial Fibrillation, Aspirin, and Anticoagulation Study. Arch Intern Med. 1998;158(14): 1513-1521.

288. Coumadin Aspirin Reinfarction Study (CARS) Investigators. Randomised, double-blind trial of fixed low-dose warfarin with aspirin after myocardial infarction. Lancet. 1997; 350(9075):389-396

289. Pengo V, Zasso A, Barbero F, et al. Effectiveness of fixed minidose warfarin in the prevention of thromboembolism and vascular death in nonrheumatic atrial fibrillation. Am J Cardiol. 1998;82(4):433-437.

290. Couban S, Goodyear M, Burnell M, et al. Randomized placebo-controlled study of low-dose warfarin for the prevention of central venous catheter-associated thrombosis in patients with cancer. J Clin Oncol. 2005;23(18): 4063-4069.

291. Garcia DA, Regan S, Crowther M, Hylek EM. The risk of hemorrhage among patients with warfarin-associated coagulopathy. J Am Coll Cardiol. 2006;47(4):804-808.

292. Clark NP, Witt DM, Delate T, et al; Warfarin-Associated Research Projects and Other Endeavors Consortium. Thromboembolic consequences of subtherapeutic anticoagulation in patients stabilized on warfarin therapy: the low INR study. Pharmacotherapy. 2008;28(8):960-967.

293. Dentali F, Riva N, Malato A, Saccullo G, Siragusa S, Ageno W. Incidence of thromboembolic complications in patients with mechanical heart valves with a subtherapeutic international normalized ratio. J Thorac Cardiovasc Surg. 2009;137(1):91-93.

294. Garcia DA, Regan S, Henault LE, et al. Risk of thromboembolism with short-term interruption of warfarin therapy. Arch Intern Med. 2008;168(1):63-69.

295. Delate T, Witt DM, Jones JR, Bhardwaja B, Senser M. Falsely elevated international normalized ratio values in patients undergoing anticoagulation therapy: a descriptive evaluation. Chest. 2007;131(3):816-822.

296. Coon WW, Willis PW III. Hemorrhagic complications of anticoagulant therapy. Arch Intern Med. 1974;133(3): 386-392.
297. Jaffin BW, Bliss CM, LaMont JT. Significance of occult gastrointestinal bleeding during anticoagulation therapy. Am J Med. 1987;83(2):269-272.

298. Wilcox CM, Truss CD. Gastrointestinal bleeding in patients receiving long-term anticoagulant therapy. Am J Med. 1988; 84(4):683-690

299. Wolf AT, Wasan SK, Saltzman JR. Impact of anticoagulation on rebleeding following endoscopic therapy for nonvariceal upper gastrointestinal hemorrhage. Am J Gastroenterol. 2007;102(2):290-296.

300. Culclasure TF, Bray VJ, Hasbargen JA. The significance of hematuria in the anticoagulated patient. Arch Intern Med. 1994; 154(6):649-652.

301. Schuster GA, Lewis GA. Clinical significance of hematuria in patients on anticoagulant therapy. J Urol. 1987;137(5): 923-925.

302. Van Savage JG, Fried FA. Anticoagulant associated hematuria: a prospective study. J Urol. 1995;153(5):1594-1596.

303. Garcia D, Ageno W, Bussey H, et al. Prevention and treatment of bleeding complications in patients receiving vitamin K antagonists, Part 1: Prevention. Am J Hematol. 2009;84(9):579-583.

304. Dahri K, Loewen P. The risk of bleeding with warfarin: a systematic review and performance analysis of clinical prediction rules. Thromb Haemost. 2007;98(5):980-987.

305. Gage BF, Yan Y, Milligan PE, et al. Clinical classification schemes for predicting hemorrhage: results from the National Registry of Atrial Fibrillation (NRAF). Am Heart J. 2006;151(3):713-719

306. Wickramasinghe LSP, Basu SK, Bansal SK. Long-term oral anticoagulant therapy in elderly patients. Age Ageing. 1988; 17(6):388-396.

307. Copland M, Walker ID, Tait RC. Oral anticoagulation and hemorrhagic complications in an elderly population with atrial fibrillation. Arch Intern Med. 2001;161(17):2125-2128.

308. Launbjerg J, Egeblad H, Heaf J, Nielsen NH, Fugleholm AM, Ladefoged K. Bleeding complications to oral anticoagulant therapy: multivariate analysis of 1010 treatment years in 551 outpatients. J Intern Med. 1991;229(4):351-355.

309. The Stroke Prevention in Atrial Fibrillation Investigators. Bleeding during antithrombotic therapy in patients with atrial fibrillation. Arch Intern Med. 1996;156(4):409-416.

310. Fihn SD, Callahan CM, Martin DC, McDonell MB, Henikoff JG, White RH; The National Consortium of Anticoagulation Clinics. The risk for and severity of bleeding complications in elderly patients treated with warfarin. Ann Intern Med. 1996;124(11):970-979.

311. Petitti DB, Strom BL, Melmon KL. Duration of warfarin anticoagulant therapy and the probabilities of recurrent thromboembolism and hemorrhage. Am J Med. 1986;81(2): 255-259.

312. McCormick D, Gurwitz JH, Goldberg RJ, Ansell J. Longterm anticoagulation therapy for atrial fibrillation in elderly patients: efficacy, risk, and current patterns of use. J Thromb Thrombolysis. 1999; 7(2):157-163.

313. Cheng HY. Is age a risk factor for warfarin-related major bleeds in elderly patients with atrial fibrillation? I Am Geriatr Soc. 2006;54(7):1155.

314. Palareti G, Hirsh J, Legnani C, et al. Oral anticoagulation treatment in the elderly: a nested, prospective, case-control study. Arch Intern Med. 2000;160(4):470-478.

315. Fang MC, Go AS, Hylek EM, et al. Age and the risk of warfarin-associated hemorrhage: the anticoagulation and risk factors in atrial fibrillation study. J Am Geriatr Soc. 2006;54(8):1231-1236.

316. Dentali F, Douketis JD, Lim W, Crowther M. Combined aspirin-oral anticoagulant therapy compared with oral 
anticoagulant therapy alone among patients at risk for cardiovascular disease: a meta-analysis of randomized trials. Arch Intern Med. 2007;167(2):117-124.

317. Flaker GC, Gruber M, Connolly SJ, et al; SPORTIF Investigators. Risks and benefits of combining aspirin with anticoagulant therapy in patients with atrial fibrillation: an exploratory analysis of stroke prevention using an oral thrombin inhibitor in atrial fibrillation (SPORTIF) trials. Am Heart J. 2006;152(5):967-973.

318. Rubboli A, Halperin JL, Airaksinen KE, et al. Antithrombotic therapy in patients treated with oral anticoagulation undergoing coronary artery stenting. An expert consensus document with focus on atrial fibrillation. Ann Med. 2008;40(6): 428-436.

319. Hansen ML, Sørensen R, Clausen MT, et al. Risk of bleeding with single, dual, or triple therapy with warfarin, aspirin, and clopidogrel in patients with atrial fibrillation. Arch Intern Med. 2010;170(16):1433-1441.

320. Ageno W, Garcia D, Aguilar MI, et al. Prevention and treatment of bleeding complications in patients receiving vitamin $\mathrm{K}$ antagonists, part 2: Treatment. Am J Hematol. 2009;84(9):584-588.

321. Patel RJ, Witt DM, Saseen JJ, Tillman DJ, Wilkinson DS. Randomized, placebo-controlled trial of oral phytonadione for excessive anticoagulation. Pharmacotherapy. 2000;20(10): 1159-1166.

322. Fondevila CG, Grosso SH, Santarelli MT, Pinto MD. Reversal of excessive oral anticoagulation with a low oral dose of vitamin K1 compared with acenocoumarine discontinuation. A prospective, randomized, open study. Blood Coagul Fibrinolysis. 2001;12(1):9-16.

323. Ageno W, Crowther M, Steidl L, et al. Low dose oral vitamin $\mathrm{K}$ to reverse acenocoumarol-induced coagulopathy: a randomized controlled trial. Thromb Haemost. 2002;88(1): 48-51.

324. Dentali F, Ageno W, Crowther M. Treatment of coumarin-associated coagulopathy: a systematic review and proposed treatment algorithms. J Thromb Haemost. 2006;4(9):1853-1863.

325. Lubetsky A, Yonath H, Olchovsky D, Loebstein R, Halkin H, Ezra D. Comparison of oral vs intravenous phytonadione (vitamin K1) in patients with excessive anticoagulation: a prospective randomized controlled study. Arch Intern Med. 2003;163(20):2469-2473.

326. Crowther MA, Douketis JD, Schnurr T, et al. Oral vitamin K lowers the international normalized ratio more rapidly than subcutaneous vitamin $\mathrm{K}$ in the treatment of warfarinassociated coagulopathy. A randomized, controlled trial. Ann Intern Med. 2002;137(4):251-254.

327. Nee R, Doppenschmidt D, Donovan DJ, Andrews TC. Intravenous versus subcutaneous vitamin K1 in reversing excessive oral anticoagulation. Am J Cardiol. 1999;83(2): 286-288; A6-A7.

328. Raj G, Kumar R, McKinney WP. Time course of reversal of anticoagulant effect of warfarin by intravenous and subcutaneous phytonadione. Arch Intern Med. 1999;159(22): 2721-2724.

329. Riegert-Johnson DL, Volcheck GW. The incidence of anaphylaxis following intravenous phytonadione (vitamin K1): a 5-year retrospective review. Ann Allergy Asthma Immunol. 2002;89(4):400-406.

330. Contreras M, Ala FA, Greaves M, et al. Guidelines for the use of fresh frozen plasma. British Committee for Standards in Haematology, Working Party of the Blood Transfusion Task Force. Transfus Med. 1992;2(1):57-63.

331. Popovsky MA. Transfusion-Related Acute Lung Injury: Incidence, Pathogenesis and the Role of Multicomponent
Apheresis in Its Prevention. Transfus Med Hemother. 2008; 35(2):76-79.

332. Leissinger CA, Blatt PM, Hoots WK, Ewenstein B. Role of prothrombin complex concentrates in reversing warfarin anticoagulation: a review of the literature. Am J Hematol. 2008;83(2):137-143

333. Rosovsky RP, Crowther MA. What is the evidence for the off-label use of recombinant factor VIIa (rFVIIa) in the acute reversal of warfarin? ASH evidence-based review 2008. Hematology Am Soc Hematol Educ Program. 2008:36-38.

334. Verhagen H. Local hemorrhage and necrosis of the skin and underlying tissues at starting therapy with dicumarol or dicumacyl. Acta Med Scand. 1954;148(6):453-467.

335. Weinberg AC, Lieskovsky G, McGehee WG, Skinner DG. Warfarin necrosis of the skin and subcutaneous tissue of the male external genitalia. J Urol. 1983;130(2):352-354.

336. Broekmans AW, Bertina RM, Loeliger EA, Hofmann V, Klingemann HG. Protein $\mathrm{C}$ and the development of skin necrosis during anticoagulant therapy. Thromb Haemost. 1983;49(3):251.

337. Zauber NP, Stark MW. Successful warfarin anticoagulation despite protein $\mathrm{C}$ deficiency and a history of warfarin necrosis. Ann Intern Med. 1986;104(5):659-660.

338. Samama M, Horellou MH, Soria J, Conard J, Nicolas G. Successful progressive anticoagulation in a severe protein $\mathrm{C}$ deficiency and previous skin necrosis at the initiation of oral anticoagulant treatment. Thromb Haemost. 1984;51(1): 132-133.

339. Grimaudo V, Gueissaz F, Hauert J, Sarraj A, Kruithof EK, Bachmann F. Necrosis of skin induced by coumarin in a patient deficient in protein S. BMJ. 1989;298(6668): 233-234.

340. Warkentin TE. Venous limb gangrene during warfarin treatment of cancer-associated deep venous thrombosis. Ann Intern Med. 2001;135(8 pt 1):589-593.

341. Warkentin TE, Elavathil LJ, Hayward CPM, Johnston MA, Russett JI, Kelton JG. The pathogenesis of venous limb gangrene associated with heparin-induced thrombocytopenia. Ann Intern Med. 1997;127(9):804-812.

342. Srinivasan AF, Rice L, Bartholomew JR, et al. Warfarininduced skin necrosis and venous limb gangrene in the setting of heparin-induced thrombocytopenia. Arch Intern Med. 2004;164(1):66-70.

343. Raj K, Collins B, Rangarajan S. Purple toe syndrome following anticoagulant therapy. Br J Haematol. 2001;114(4):740.

344. Talmadge DB, Spyropoulos AC. Purple toes syndrome associated with warfarin therapy in a patient with antiphospholipid syndrome. Pharmacotherapy. 2003;23(5):674-677.

345. Hauschka PV, Lian JB, Cole DEC, Gundberg CM. Osteocalcin and matrix Gla protein: vitamin K-dependent proteins in bone. Physiol Rev. 1989;69(3):990-1047.

346. Price PA. Role of vitamin K-dependent proteins in bone metabolism. Ann Rev Nutr. 1988;8:565-583.

347. Maillard C, Berruyer M, Serre CM, Dechavanne M, Delmas PD. Protein-S, a vitamin K-dependent protein, is a bone matrix component synthesized and secreted by osteoblasts. Endocrinology. 1992;130(3):1599-1604.

348. Pan LC, Williamson MK, Price PA. Sequence of the precursor to rat bone gamma-carboxyglutamic acid protein that accumulates in warfarin-treated osteosarcoma cells. J Biol Chem. 1985;260(25):13398-13401.

349. Pettifor JM, Benson R. Congenital malformations associated with the administration of oral anticoagulants during pregnancy. J Pediatr. 1975;86(3):459-462.

350. Hall JG, Pauli RM, Wilson KM. Maternal and fetal sequelae of anticoagulation during pregnancy. Am J Med. 1980;68(1): 122-140. 
351. Barnes C, Newall F, Ignjatovic V, et al. Reduced bone density in children on long-term warfarin. Pediatr Res. 2005; 57(4):578-581.

352. Danziger J. Vitamin K-dependent proteins, warfarin, and vascular calcification. Clin J Am Soc Nephrol. 2008;3(5): 1504-1510.

353. Lazo-Langner A, Rodger MA, Wells PS. Lessons from ximelagatran: issues for future studies evaluating new oral direct thrombin inhibitors for venous thromboembolism prophylaxis in orthopedic surgery. Clin Appl Thromb Hemost. 2009;15(3):316-326.

354. Diener HC; Executive Steering Committee of the SPORTIFF III and V Investigators. Stroke prevention using the oral direct thrombin inhibitor ximelagatran in patients with nonvalvular atrial fibrillation. Pooled analysis from the SPORTIF III and V studies. Cerebrovasc Dis. 2006;21(4):279-293.

355. Boudes PF. The challenges of new drugs benefits and risks analysis: lessons from the ximelagatran FDA Cardiovascular Advisory Committee. Contemp Clin Trials. 2006;27(5): 432-440.

356. Nutescu EA, Shapiro NL, Chevalier A. New anticoagulant agents: direct thrombin inhibitors. Cardiol Clin. 2008;26(2): 169-187; v-vi.

357. Hauel NH, Nar H, Priepke H, Ries U, Stassen JM, Wienen W. Structure-based design of novel potent nonpeptide thrombin inhibitors. J Med Chem. 2002;45(9):1757-1766.

358. Wienen W, Stassen JM, Priepke H, Ries UJ, Hauel N. In-vitro profile and ex-vivo anticoagulant activity of the direct thrombin inhibitor dabigatran and its orally active prodrug, dabigatran etexilate. Thromb Haemost. 2007;98(1): 155-162.

359. Blech S, Ebner T, Ludwig-Schwellinger E, Stangier J, Roth W. The metabolism and disposition of the oral direct thrombin inhibitor, dabigatran, in humans. Drug Metab Dispos. 2008;36(2):386-399.

360. Lange U, Nowak G, Bucha E. Ecarin chromogenic assay-a new method for quantitative determination of direct thrombin inhibitors like hirudin. Pathophysiol Haemost Thromb. 2003;33(4):184-191.

361. Stangier J, Rathgen K, Stähle H, Gansser D, Roth W. The pharmacokinetics, pharmacodynamics and tolerability of dabigatran etexilate, a new oral direct thrombin inhibitor, in healthy male subjects. Br J Clin Pharmacol. 2007;64(3): 292-303.

362. Wienen W, Stassen JM, Priepke H, Ries UJ, Hauel N. Effects of the direct thrombin inhibitor dabigatran and its orally active prodrug, dabigatran etexilate, on thrombus formation and bleeding time in rats. Thromb Haemost. 2007; 98(2):333-338.

363. Wienen W, Stassen JM, Priepke H, Ries UJ, Hauel N. Antithrombotic and anticoagulant effects of the direct thrombin inhibitor dabigatran, and its oral prodrug, dabigatran etexilate, in a rabbit model of venous thrombosis. J Thromb Haemost. 2007;5(6):1237-1242.

364. Stangier J, Stähle H, Rathgen K, Fuhr R. Pharmacokinetics and pharmacodynamics of the direct oral thrombin inhibitor dabigatran in healthy elderly subjects. Clin Pharmacokinet. 2008;47(1):47-59.

365. Stangier J, Eriksson BI, Dahl OE, et al. Pharmacokinetic profile of the oral direct thrombin inhibitor dabigatran etexilate in healthy volunteers and patients undergoing total hip replacement. J Clin Pharmacol. 2005;45(5):555-563.

366. Trocóniz IF, Tillmann C, Liesenfeld KH, Schäfer HG, Stangier J. Population pharmacokinetic analysis of the new oral thrombin inhibitor dabigatran etexilate (BIBR 1048) in patients undergoing primary elective total hip replacement surgery. J Clin Pharmacol. 2007;47(3):371-382.
367. Stangier J. Clinical pharmacokinetics and pharmacodynamics of the oral direct thrombin inhibitor dabigatran etexilate. Clin Pharmacokinet. 2008;47(5):285-295.

368. Eriksson BI, Dahl OE, Ahnfelt L, et al. Dose escalating safety study of a new oral direct thrombin inhibitor, dabigatran etexilate, in patients undergoing total hip replacement: BISTRO I. J Thromb Haemost. 2004;2(9):1573-1580.

369. van Ryn J, Stangier J, Haertter S, et al. Dabigatran etexilatea novel, reversible, oral direct thrombin inhibitor: interpretation of coagulation assays and reversal of anticoagulant activity. Thromb Haemost. 2010;103(6):1116-1127.

370. Stangier J, Rathgen K, Stähle H, Mazur D. Influence of renal impairment on the pharmacokinetics and pharmacodynamics of oral dabigatran etexilate: an open-label, parallel-group, single-centre study. Clin Pharmacokinet. 2010; 49(4):259-268.

371. Stangier J, Stähle H, Rathgen K, Roth W, Shakeri-Nejad K. Pharmacokinetics and pharmacodynamics of dabigatran etexilate, an oral direct thrombin inhibitor, are not affected by moderate hepatic impairment. J Clin Pharmacol. 2008; 48(12):1411-1419.

372. Ezekowitz MD, Reilly PA, Nehmiz G, et al. Dabigatran with or without concomitant aspirin compared with warfarin alone in patients with nonvalvular atrial fibrillation (PETRO Study). Am J Cardiol. 2007;100(9):1419-1426.

373. EMEA. CHMP assessment report for Pradaxa. http://www. emaeuropaeu/humandocs/PDFs/EPAR/pradaxa/H-829en6pdf. 2008. Accessed March 2, 2011.

374. Mouly S, Meune C, Bergmann JF. Mini-series: I. Basic science. Uncertainty and inaccuracy of predicting CYP-mediated in vivo drug interactions in the ICU from in vitro models: focus on CYP3A4. Intensive Care Med. 2009;35(3):417-429.

375. Stangier J, Rathgen K, Stähle H, Reseski K, Körnicke T, Roth W. Coadministration of dabigatran etexilate and atorvastatin: assessment of potential impact on pharmacokinetics and pharmacodynamics. Am J Cardiovasc Drugs. 2009;9(1):59-68

376. Stangier J, Stähle H, Rathgen K, Reseski K, Körnicke T. Coadministration of the oral direct thrombin inhibitor dabigatran etexilate and diclofenac has little impact on the pharmacokinetics of either drug [abstract]. J Thromb Haemost. 2007;5(suppl 2):P-T-677.

377. Marchetti S, Mazzanti R, Beijnen JH, Schellens JHM. Concise review: Clinical relevance of drug drug and herb drug interactions mediated by the ABC transporter ABCB1 (MDR1, P-glycoprotein). Oncologist. 2007;12(8):927-941.

378. Stangier J, Stähle H, Rathgen K, Reseski K, Körnicke T. No interaction of the oral direct thrombin inhibitor dabigatran etexilate and digoxin [abstract]. J Thromb Haemost. 2007;5(suppl 2):P-W-672.

379. Patrono C, García Rodríguez LA, Landolfi R, Baigent C. Low-dose aspirin for the prevention of atherothrombosis. N Engl J Med. 2005;353(22):2373-2383.

380. Holmes DR Jr, Kereiakes DJ, Kleiman NS, Moliterno DJ, Patti G, Grines CL. Combining antiplatelet and anticoagulant therapies. J Am Coll Cardiol. 2009;54(2):95-109.

381. Connolly SJ, Ezekowitz MD, Yusuf S, et al; RE-LY Steering Committee and Investigators. Dabigatran versus warfarin in patients with atrial fibrillation. N Engl J Med. 2009; 361(12):1139-1151.

382. Schulman S, Kearon C, Kakkar AK, et al; RE-COVER Study Group. Dabigatran versus warfarin in the treatment of acute venous thromboembolism. N Engl J Med. 2009; 361(24):2342-2352.

383. Eriksson BI, Dahl OE, Rosencher N, et al; RE-NOVATE Study Group. Dabigatran etexilate versus enoxaparin for prevention of venous thromboembolism after total hip 
replacement: a randomised, double-blind, non-inferiority trial. Lancet. 2007;370(9591):949-956.

384. Eriksson BI, Dahl OE, Rosencher N, et al; RE-MODEL Study Group. Oral dabigatran etexilate vs. subcutaneous enoxaparin for the prevention of venous thromboembolism after total knee replacement: the RE-MODEL randomized trial. J Thromb Haemost. 2007;5(11):2178-2185.

385. Ginsberg JS, Davidson BL, Comp PC, et al; RE-MOBILIZE Writing Committee. Oral thrombin inhibitor dabigatran etexilate vs North American enoxaparin regimen for prevention of venous thromboembolism after knee arthroplasty surgery. J Arthroplasty. 2009;24(1):1-9.

386. Huo MH, Eriksson B, Dahl OE, et al. Oral dabigatran versus enoxaparin for thromboprophylaxis after primary total hip arthroplasty: the RE-NOVATE II randomised trial. Presented at: The European Hematology Association Congress; June 10-13, 2010; Barcelona, Spain.

387. Singer DE, Albers GW, Dalen JE, et al; American College of Chest Physicians. Antithrombotic therapy in atrial fibrillation: American College of Chest Physicians Evidence-Based Clinical Practice Guidelines (8th Edition). Chest. 2008; 133(suppl 6):546S-592S.

388. Schulman S, Beyth RJ, Kearon C, Levine MN; American College of Chest Physicians. Hemorrhagic complications of anticoagulant and thrombolytic treatment: American College of Chest Physicians Evidence-Based Clinical Practice Guidelines(8th Edition).Chest.2008;133(suppl6): 257S-298S.

389. Mismetti P, Laporte S. New oral antithrombotics: a need for laboratory monitoring. For. J Thromb Haemost. 2010; $8(4): 621-626$.

390. Bounameaux H, Reber G. New oral antithrombotics: a need for laboratory monitoring. Against. J Thromb Haemost. 2010; 8(4):627-630.

391. Keisu M, Andersson TB. Drug-induced liver injury in humans: the case of ximelagatran. Handb Exp Pharmacol. 2010;196:407-418.

392. Agnelli G, Eriksson BI, Cohen AT, et al; EXTEND Study Group. Safety assessment of new antithrombotic agents: lessons from the EXTEND study on ximelagatran. Thromb Res. 2009;123(3):488-497.

393. Eriksson BI, Borris LC, Friedman RJ, et al; RECORD1 Study Group. Rivaroxaban versus enoxaparin for thromboprophylaxis after hip arthroplasty. N Engl J Med. 2008; 358(26):2765-2775

394. Kakkar AK, Brenner B, Dahl OE, et al; RECORD2 Investigators. Extended duration rivaroxaban versus short-term enoxaparin for the prevention of venous thromboembolism after total hip arthroplasty: a double-blind, randomised controlled trial. Lancet. 2008;372(9632):31-39.

395. Lassen MR, Ageno W, Borris LC, et al; RECORD3 Investigators. Rivaroxaban versus enoxaparin for thromboprophylaxis after total knee arthroplasty. N Engl J Med. 2008; 358(26):2776-2786.

396. Turpie AG, Lassen MR, Davidson BL, et al; RECORD4 Investigators. Rivaroxaban versus enoxaparin for thromboprophylaxis after total knee arthroplasty (RECORD4): a randomised trial. Lancet. 2009;373(9676):1673-1680.

397. Graff J, von Hentig N, Misselwitz F, et al. Effects of the oral, direct factor xa inhibitor rivaroxaban on platelet-induced thrombin generation and prothrombinase activity. J Clin Pharmacol. 2007;47(11):1398-1407.

398. Perzborn E, Roehrig S, Straub A, Kubitza D, Mueck W, Laux V. Rivaroxaban: a new oral factor Xa inhibitor. Arterioscler Thromb Vasc Biol. 2010;30(3):376-381.

399. Perzborn E, Strassburger J, Wilmen A, et al. In vitro and in vivo studies of the novel antithrombotic agent BAY 59-
7939 — an oral, direct Factor Xa inhibitor. J Thromb Haemost. 2005;3(3):514-521.

400. Gerotziafas GT, Elalamy I, Depasse F, Perzborn E, Samama MM. In vitro inhibition of thrombin generation, after tissue factor pathway activation, by the oral, direct factor Xa inhibitor rivaroxaban. J Thromb Haemost. 2007;5(4):886-888.

401. Lang D, Freudenberger C, Weinz C. In vitro metabolism of rivaroxaban, an oral, direct factor Xa inhibitor, in liver microsomes and hepatocytes of rats, dogs, and humans. Drug Metab Dispos. 2009;37(5):1046-1055.

402. Samama MM, Martinoli JL, LeFlem L, et al. Assessment of laboratory assays to measure rivaroxaban-an oral, direct factor Xa inhibitor. Thromb Haemost. 2010;103(4): 815-825.

403. Kubitza D, Becka M, Mueck W, Zuehlsdorf M. Safety, tolerability, pharmacodynamics, and pharmacokinetics of rivaroxaban - an oral, direct factor Xa inhibitor-are not affected by aspirin. J Clin Pharmacol. 2006;46(9):981-990.

404. Kubitza D, Becka M, Voith B, Zuehlsdorf M, Wensing G. Safety, pharmacodynamics, and pharmacokinetics of single doses of BAY 59-7939, an oral, direct factor Xa inhibitor. Clin Pharmacol Ther. 2005;78(4):412-421.

405. Kubitza D, Becka M, Wensing G, Voith B, Zuehlsdorf M. Safety, pharmacodynamics, and pharmacokinetics of BAY 59-7939 — an oral, direct Factor Xa inhibitor-after multiple dosing in healthy male subjects. Eur J Clin Pharmacol. 2005;61(12):873-880.

406. Mueck W, Becka M, Kubitza D, Voith B, Zuehlsdorf M. Population model of the pharmacokinetics and pharmacodynamics of rivaroxaban — an oral, direct factor xa inhibitor - in healthy subjects. Int J Clin Pharmacol Ther. 2007;45(6): 335-344.

407. Kubitza D, Becka M, Roth A, Mueck W. Dose-escalation study of the pharmacokinetics and pharmacodynamics of rivaroxaban in healthy elderly subjects. Curr Med Res Opin. 2008;24(10):2757-2765.

408. Kubitza D, Becka M, Zuehlsdorf M, Mueck W. Body weight has limited influence on the safety, tolerability, pharmacokinetics, or pharmacodynamics of rivaroxaban (BAY 59-7939) in healthy subjects. J Clin Pharmacol. 2007;47(2): 218-226.

409. Kubitza D, Becka M, Mueck W, Zuehlsdorf M. The effect of extreme age, and gender, on the pharmacology and tolerability of rivaroxaban-an oral, direct Factor Xa inhibitor [abstract]. Blood. 2006;108:905.

410. Jiang J, Hu Y, Zhang J, et al. Safety, pharmacokinetics and pharmacodynamics of single doses of rivaroxaban - an oral, direct factor Xa inhibitor-in elderly Chinese subjects. Thromb Haemost. 2010;103(1):234-241.

411. Zhao X, Sun P, Zhou Y, et al. Safety, pharmacokinetics and pharmacodynamics of single/multiple doses of the oral, direct Factor Xa inhibitor rivaroxaban in healthy Chinese subjects. Br J Clin Pharmacol. 2009;68(1):77-88.

412. Mueck W, Eriksson BI, Bauer KA, et al. Population pharmacokinetics and pharmacodynamics of rivaroxaban-an oral, direct factor $\mathrm{Xa}$ inhibitor-in patients undergoing major orthopaedic surgery. Clin Pharmacokinet. 2008;47(3): 203-216.

413. Mueck W, Borris LC, Dahl OE, et al. Population pharmacokinetics and pharmacodynamics of once- and twice-daily rivaroxaban for the prevention of venous thromboembolism in patients undergoing total hip replacement. Thromb Haemost. 2008;100(3):453-461.

414. Bayer Schering Pharma. Xarelto. Summary of Product Characteristics. May 2009. www.xarelto.com/html/downloads/ Xarelto_Summary_of_Product_Characteristics_May2009.pdf. Accessed March 2, 2011. 
415. Kubitza D, Mueck W, Becka M. No interaction between rivaroxaban - a novel, oral, direct factor Xa inhibitor-and atorvastatin [abstract]. Pathophysiol Haemost Thromb. 2008; 36:A40.

416. Kubitza D, Becka M, Zuehlsdorf M. No interaction between the novel, oral direct Factor Xa inhibitor BAY 59-7939 and digoxin [abstract]. J Clin Pharmacol. 2006; 46(6):11.

417. Kubitza D, Becka M, Mueck W, Zuehlsdorf M. Rivaroxaban (BAY 59-7939) — an oral, direct Factor Xa inhibitor-has no clinically relevant interaction with naproxen. $\mathrm{Br}$ J Clin Pharmacol. 2007;63(4):469-476.

418. Kubitza D, Becka M, Zuehlsdorf M, Mueck W. Effect of food, an antacid, and the H2 antagonist ranitidine on the absorption of BAY 59-7939 (rivaroxaban), an oral, direct factor Xa inhibitor, in healthy subjects. J Clin Pharmacol. 2006;46(5):549-558.

419. Tersteegen A, Schmidt S, Burkhardt N. Rivaroxaban-an oral, direct Factor Xa inhibitor - binds rapidly to Factor Xa [abstract]. J Thromb Haemost. 2007;5(Suppl 2):651.

420. Depasse F, Busson J, Mnich J, Le Flem L, Gerotziafas GT, Samama MM. Effect of BAY 59-7939-a novel, oral, direct Factor Xa inhibitor-on clot-bound Factor Xa activity in vitro [abstract]. J Thromb Haemost. 2005;3(suppl 1):P1104.

421. Perzborn E, Strassburger J, Wilmen A, et al. Biochemical and pharmacologic properties of BAY 59-7939, an oral, direct Factor Xa inhibitor [abstract]. Pathophysiol Haemost Thromb. 2004;33(suppl 2):79.

422. Hoppensteadt D, Neville B, Schultz C, et al. Interaction of BAY 59-7939 - a novel, oral, direct Factor Xa inhibitor- with antiplatelet agents: monitoring and therapeutic applications [abstract]. J Thromb Haemost. 2005;3(suppl 1):1717.

423. Perzborn E, Lange U. Rivaroxaban—an oral, direct Factor Xa inhibitor- inhibits tissue factor-mediated platelet aggregation [abstract]. J Thromb Haemost. 2007;5(suppl 2):642.

424. Smith SA, Morrissey JH. Thromboplastin composition affects the sensitivity of prothrombin time clotting tests to direct Factor Xa inhibitors [abstract]. Blood. 2007;110(11):928.

425. Bauersachs R, Berkowitz SD, Brenner B, et al; EINSTEIN Investigators. Oral rivaroxaban for symptomatic venous thromboembolism. N Engl J Med. 2010;363(26):2499-2510.

426. Patel MR, Mahaffey KW, Garg J, et al; ROCKET AF Investigators. Rivaroxaban versus warfarin in nonvalvular atrial fibrillation. N Engl J Med. 2011;365(10):883-891.

427. National Institutes of Health Clinical Center. An efficacy and safety study for rivaroxaban in patients with acute coronary syndrome. NCT00809965. ClinicTrials.gov. Bethesda, MD: National Institutes of Health; 2008. Updated 2011. www.clinicaltrials.gov.

428. Turpie AG, Lassen MR, Eriksson BI, et al. Rivaroxaban for the prevention of venous thromboembolism after hip or knee arthroplasty. Pooled analysis of four studies. Thromb Haemost. 2011;105(3):444-453.

429. Perzborn E, Tinel H. Prothrombin complex concentrate reverses the effects of high-dose rivaroxaban in rats [abstract]. J Thromb Haemost. 2009; 7(suppl 2):183.

430. Lu G, Luan P, Hollenbach SJ, et al. Reconstructed recombinant factor $\mathrm{Xa}$ as an antidote to reverse anticoagulation by factor Xa inhibitors [abstract]. J Thromb Haemost. 2009; 7(suppl 2):OC-TH-107. 